\title{
Adolescent chronic pain
}

Citation for published version (APA):

van den Bogert-Dekker, C. (2018). Adolescent chronic pain: Rehabilitation treatment targeting painrelated fear. [Doctoral Thesis, Maastricht University]. Maastricht University. https://doi.org/10.26481/dis.20181123cb

Document status and date:

Published: 01/01/2018

DOI:

10.26481/dis.20181123cb

Document Version:

Publisher's PDF, also known as Version of record

\section{Please check the document version of this publication:}

- A submitted manuscript is the version of the article upon submission and before peer-review. There can be important differences between the submitted version and the official published version of record.

People interested in the research are advised to contact the author for the final version of the publication, or visit the DOI to the publisher's website.

- The final author version and the galley proof are versions of the publication after peer review.

- The final published version features the final layout of the paper including the volume, issue and page numbers.

Link to publication

\footnotetext{
General rights rights.

- You may freely distribute the URL identifying the publication in the public portal. please follow below link for the End User Agreement:

www.umlib.nl/taverne-license

Take down policy

If you believe that this document breaches copyright please contact us at:

repository@maastrichtuniversity.nl

providing details and we will investigate your claim.
}

Copyright and moral rights for the publications made accessible in the public portal are retained by the authors and/or other copyright owners and it is a condition of accessing publications that users recognise and abide by the legal requirements associated with these

- Users may download and print one copy of any publication from the public portal for the purpose of private study or research.

- You may not further distribute the material or use it for any profit-making activity or commercial gain

If the publication is distributed under the terms of Article $25 \mathrm{fa}$ of the Dutch Copyright Act, indicated by the "Taverne" license above, 


\section{Adolescent chronic pain}

Rehabilitation treatment targeting pain-related fear

Carolien van den Bogert-Dekker 
(c) 2018 C. van den Bogert-Dekker, Heerlen, The Netherlands

ISBN: 978-90-829290-0-3

Cover design: Maartje Dekker

Typesetting: *studio Michal Slawinski, thesisprint.eu

Printed in Poland

The copyright of the published articles in this dissertation has been transferred to the respective journals. All rights reserved. No part of this publication may be reproduced or transmitted in any form or by any means, electronical or mechanical, including photocopy, recording or any other information storage or retrieval system, without prior written permission of the copyright owner.

The research presented in this dissertation was conducted at CAPHRI Care and Public Health Research Institute, Department of Rehabilitation Medicine, of Maastricht University. The 2B Active project was funded by Fonds Nuts Ohra (1202-068), Adelante, Center of Expertise in Rehabilitation and Audiology Hoensbroek, and Stichting Vooruit. 


\title{
Adolescent chronic pain \\ Rehabilitation treatment targeting pain-related fear
}

\author{
Proefschrift
}

ter verkrijging van de graad van doctor aan de Universiteit Maastricht, op gezag van de Rector Magnificus,

Prof. dr. Rianne M. Letschert

volgens het besluit van het College van Decanen,

in het openbaar te verdedigen

op vrijdag 23 november 2018 om 12:00 uur

door

Carolien van den Bogert-Dekker 


\section{Promotor}

Prof. dr. J.A.M.C.F. Verbunt

\section{Copromotores}

Dr. M.E.J.B. Goossens

Dr. C.H.G. Bastiaenen

\section{Beoordelingscommissie}

Prof. dr. A.E.R.C.H. Boonen (voorzitter)

Prof. dr. M.L. Peters

Prof. Dr. R.W.J.G. Ostelo (Vrije Universiteit Amsterdam)

Dr. rer. nat. J. Wager (Universitat Witten/Herdecke, Germany) 


\section{Table of contents}

\section{Chapter 1}

General Introduction

\section{Chapter 2}

Study protocol for a multicentre randomized controlled trial on effectiveness of an outpatient multimodal rehabilitation program* for adolescents with chronic musculoskeletal pain (2B Active)

\section{Chapter 3}

Functional disability in adolescents with chronic pain:

comparing an interdisciplinary exposure program to usual care A randomized clinical trial

\section{Chapter 4}

Pain-related fear in adolescents with chronic musculoskeletal pain:

process evaluation of an interdisciplinary graded exposure program

\section{Chapter 5}

Dutch version of the fear of pain questionnaire

for adolescents with chronic pain

\section{Chapter 6}

General discussion

\section{Chapter 7}

Valorisation addendum

Summary

Samenvatting

Dankwoord

About the author 




\section{GENERAL INTRODUCTION}

Pain is one of the most common sensations people of all ages experience in everyday life. The ability to feel pain is vital because it protects against an imminent or actual threat of body damage. In case of such a threat, people can react with a defensive response, for example by withdrawing a hand from a fire to prevent a burn, or by avoiding the use of a leg to allow a broken bone to heal.

However, in some people, acute pain turns into chronic pain when the pain persists 'beyond the expected period of healing, often chosen to be a period of three months (1). In chronic pain, the link between pain and damage to the body is not evident anymore. Paradoxically, people's normal responses in a reaction to acute pain, actually lead to detrimental effects on their health and health-related quality of life in the long term in the context of chronic pain.

Chronic pain in adults has been extensively studied. Nowadays, chronic pain in children and adolescents is increasingly recognized as a common and serious health problem. Lessons learned from research in adults with chronic pain might be applicable to the younger age group of adolescents. However, the adolescent population has its different and unique characteristics compared to the adult population, e.g. the importance of the parents in the context of adolescent chronic pain development and management (2-4), the importance of developmental stage in relation to chronic pain management (5), and influence of chronic pain on school functioning $(6,7)$ to name a few. Therefore, it is important to study this age group on its own (8). Thereby considering if and how knowledge on chronic pain assessment and management derived from adult research can be applied in adolescents. Therefore, in particular chronic pain rehabilitation treatment in adolescents, and related issues are studied in this dissertation.

\section{Epidemiology of chronic pain in childhood and adolescence}

Chronic pain is a major health concern in the pediatric population (9-11). In reports of prevalence rates of chronic pain in youth, usually child and adolescent populations are combined. Chronic headache, abdominal pain, and musculoskeletal pain are among the most common chronic pain conditions in youth $(9,12,13)$. Internationally, the most frequently studied pain type is chronic headache in youth, with an estimated median prevalence rate of 23\%. Other types of chronic pain, such as abdominal pain, back pain, musculoskeletal pain and pain combinations have been studied less frequently, although, median prevalence rates of 11\%-38\% have been reported (11). Most types of pain were found to be more prevalent in girls than in boys, with prevalence rates increasing with age (11). In the Netherlands, up to 25\% of Dutch schoolchildren (0-18 years) report pain for a period of 3 months or longer (9). Here, most frequently reported were headache, abdominal pain, limb pain and multiple pains. Furthermore, chronic pain was reported more frequent in girls, with peak prevalence rates between the ages 12-16 years (9). With regard to the severity of the pain complaints, Huguet \& Miro (14) report that $5 \%$ of the schoolchildren (8-16 years, multiple chronic pain problems, including headache, abdominal pain, limb pain and back pain) have moderate to severe chronic pain problems, meaning that these children experience 
high pain intensity and moderate to high pain-related disability. With regard to the duration of the complaints, persistence rates of pain up to 30-64\% after 4 years have been reported $(15,16)$. Additionally, pain in adolescence increases the risk of pain in adulthood $(17,18)$.

Whereas ear and abdominal pain appear to be most prevalent in young children (0-7 years), prevalence rates of headache, back pain and especially limb pain are the highest in older children and adolescents (8-18 years) (9).There has been a relative paucity in research on management of musculoskeletal pain and related disability in adolescents $(19,20)$. However, efforts have been made to develop theoretical insights into the working mechanisms for the development and maintenance of adolescent chronic musculoskeletal pain and opportunities to target treatment approaches. The focus of this dissertation is on treatment of chronic musculoskeletal pain in adolescents, defined as pain of a musculoskeletal origin and includes limb pain, back pain, Complex Regional Pain Syndrome, Complaints in Arm, Neck and Shoulder, Whiplash Associated Disorder, fibromyalgia, and joint hypermobility syndrome. It was decided that the duration of complaints should exceed 3 months in order to be classified as chronic musculoskeletal pain.

\section{Impact of adolescent chronic pain}

To understand what impact chronic musculoskeletal pain can have on the lives of adolescents suffering from these complaints, reports of only frequency and intensity of the pain provide an incomplete picture $(14,21)$. Chronic musculoskeletal pain has a wide impact, that makes it clear that there is a complex relation between chronic musculoskeletal pain and its impact on the daily lives of the adolescents (22).The complexity of the chronic pain experience in adolescents is best understood when approached from a biopsychosocial perspective. The biopsychosocial model is a heuristic approach to chronic pain and incorporates physiological, psychological as well as social factors that influence chronic pain and accompanying disability (23).

For many children and adolescents with chronic pain, their level of pain-related disability is rather low and quality of life has been found to remain relatively high when compared to patients in clinical populations (24-28). Although these children and adolescents are able to cope quite well with their complaints, in others, diverse physiological, psychological and social factors have been identified that contribute to the exacerbation and maintenance of the pain and accompanying disability. These contributing factors ultimately can result in negative outcomes for the adolescents, affecting different domains of daily living and development.

Children and adolescents (8-18 years) with musculoskeletal pain reported the severest impairments in physical functioning compared to patients with headache or abdominal pain (29). In patients referred to specialized care substantial impairments have been reported, such as school absence, less contact with friends, disturbed sleep, and having to reduce, change or even quit sports activities (29). Change in social functioning, described as changes in the contact with peers, was affected after sports and school activities were affected (29). Disturbances in school functioning have been reported in other studies as well. For example, Logan and colleagues (7) report significant school absence and a decline in grades in adolescents with chronic pain referred to tertiary care. In a study reporting on the relationship between pain severity and 
school functioning, greater pain severity was found to be associated with school absence, increased school-related pressure, decreased school-related satisfaction and increased bullying experiences (6).

Furthermore, chronic pain has also been reported to affect general emotional well-being, in terms of elevated levels of emotional distress, anxiety, and symptoms of depression $(25,30,31)$. Cohen and colleagues (32) reported that highly anxious adolescents (10-18 years old) reported poor physical functioning, high school absence and higher health care utilization, irrespective of pain intensity. Additionally, the detrimental effect of pain-related fear with associated activity avoidance and pain-related disability in adolescents with chronic pain is increasingly recognized (2, 33-36). It has even been estimated that fear of pain accounts for $40 \%$ of the variance in pain-related disability (37). It is therefore worthwhile to develop an intervention specifically targeting fear of pain in order to decrease pain-related disability.

\section{Impact on the family}

The impact of adolescent chronic pain is not restricted to the personal suffering of the adolescent but the impact is also felt in the adolescent's family, especially in the social, relational, emotional and financial domains (38). For example, in parents of adolescents with severe chronic pain, high levels of depressive symptoms, high levels of general anxiety, and high stress associated with parenting a 'difficult child' have been reported (25). Parents have been reported to take extra days off work to care for their adolescent (or even giving up or changing jobs), and to provide additional hours of care (39). Another study reported that parents of adolescents with chronic pain experience more restrictions in social life and more problems with coping with the adolescent's pain (21). Family functioning, in terms of less cohesion, and more conflict, was found to be poorer in families of children and adolescents with chronic pain compared to families of healthy children and adolescents (40).

Simultaneous to being affected by the adolescents pain, parents have a strong influence on adolescents development and maintenance of pain behavior (41), and experience of pain and disability (42). For example, Lynch-Jordan and colleagues (43) showed that parental catastrophizing (a maladaptive coping strategy for parents who have an adolescent with chronic pain) is related to poorer adolescent outcomes. Similar results were reported in a study that revealed that parents with a high level of catastrophic thinking about their child's pain experienced more distress and a greater behavioral tendency to stop their child's pain inducing activity (44).

\section{Societal impact of adolescent chronic pain}

Additionally, society bears the economic consequences, and these have been estimated to be substantial $(39,45,46)$. Direct costs involved in adolescent chronic pain are a consequence of health care expenditures. In 2001, Perquin and colleagues (47) reported that of Dutch children (0-18 years) with chronic pain, $31.1 \%$ consulted a general practitioner, $13.9 \%$ consulted a specialist, and $53.4 \%$ used pain medication. Until today, two studies aimed to estimate total costs related 
to adolescent chronic pain. Both studies accounted for the direct costs in terms of health care expenditures and indirect costs in terms of productivity losses of parents due to work absence. In 2005, Sleed and colleagues (39) estimated the mean cost per adolescent experiencing chronic pain $£ 8000$ per year in the UK $(\$ 16,400$ when converted to 2012 US dollars (46). These costs were extrapolated to a cost-of-illness to the UK society of approximately $£ 3840$ million per year ( $\$ 9.5$ billion when converted to 2012 US dollars). In 2014, Groenewald and colleagues (45) estimated the mean cost per adolescent experiencing chronic pain \$11,787 per year in the USA (2012 US dollars). These costs were extrapolated to a cost-of-illness for the USA society of approximately \$19.5 billion per year (2012 US dollars). These costs may, however, be overestimated, since both studies included small samples of adolescents with severe chronic pain (52 and 149 adolescents respectively) for which multidisciplinary tertiary treatment was sought. Nevertheless, costs are substantial. Chronic pain in adulthood even appears to be one of the most costly conditions in western society $(48,49)$.

\section{Treatment for adolescents with chronic musculoskeletal pain}

Even though the cause of chronic musculoskeletal pain often remains unexplained (50), the consequences broaden in the domains of physical, social and psychological functioning. Patients and families often become frustrated when an underlying cause for the complaints cannot be found. Simons and Basch (22) have provided an overview of different approaches for the management of chronic pain in their state of the art article, indicating that psychological treatments using cognitive-behavioral therapy and multidisciplinary treatment programs show positive results. Even without an underlying cause, there are treatment possibilities to ameliorate the negative consequences of the pain complaints. Treatments then typically focus on returning to age-appropriate functioning of the adolescent, despite the presence of their pain $(22,51)$.

For previous studies of psychological therapies in youth with headache, abdominal pain and fibromyalgia, only small and non-significant effects were found for improvements in functional disability and emotional functioning (19). In a systematic review of youth with multiple chronic pain conditions, it was concluded that multidisciplinary pain treatment may be effective in reducing disability. However, these results need to be interpreted with caution, as most of the evidence came from non-randomized studies (20). These reviews also show that there is currently no treatment specifically targeting pain-related fear in order to decrease disability in adolescents with chronic musculoskeletal pain.

The interpersonal fear avoidance model of adolescent chronic pain and graded exposure treatment

One particular characteristic in adolescent chronic pain that can potentially be targeted in treatment is pain-related fear $(32-35,37)$. As a result of emerging evidence on the role of pain-related fear in youth, the interpersonal fear avoidance model (IFAM) was developed (2). The IFAM is based on the Fear Avoidance Model by Vlaeyen \& Linton (52), the explanatory model for disability due to pain-related fear in adult chronic pain. According to the fear avoidance model (Figure 1), on the 
level of the individual patient in the event of pain, pain can be perceived as a threat, and pain-related fear can evolve. Fearful patients anticipate (re) injury to their body when executing certain movements or behaviors. Consequently, pain-related fear and catastrophic thinking cause avoidance of physical and social activities, which in the long term results in functional disability, depression and disuse (53-55), further fueling the vicious circle of chronic disabling pain. The IFAM adds to the original fear avoidance model because it incorporates the social context in which adolescent pain takes place.

To reverse the impact of pain-related fear, graded exposure in vivo has been used and studied in adults (56), with positive effects on the reduction of disability (57-59). Graded exposure in vivo is a cognitive behavioral treatment that targets pain-related fear and disability through exposing patients to activities and situations previously avoided because of fear of pain or re-injury (57).

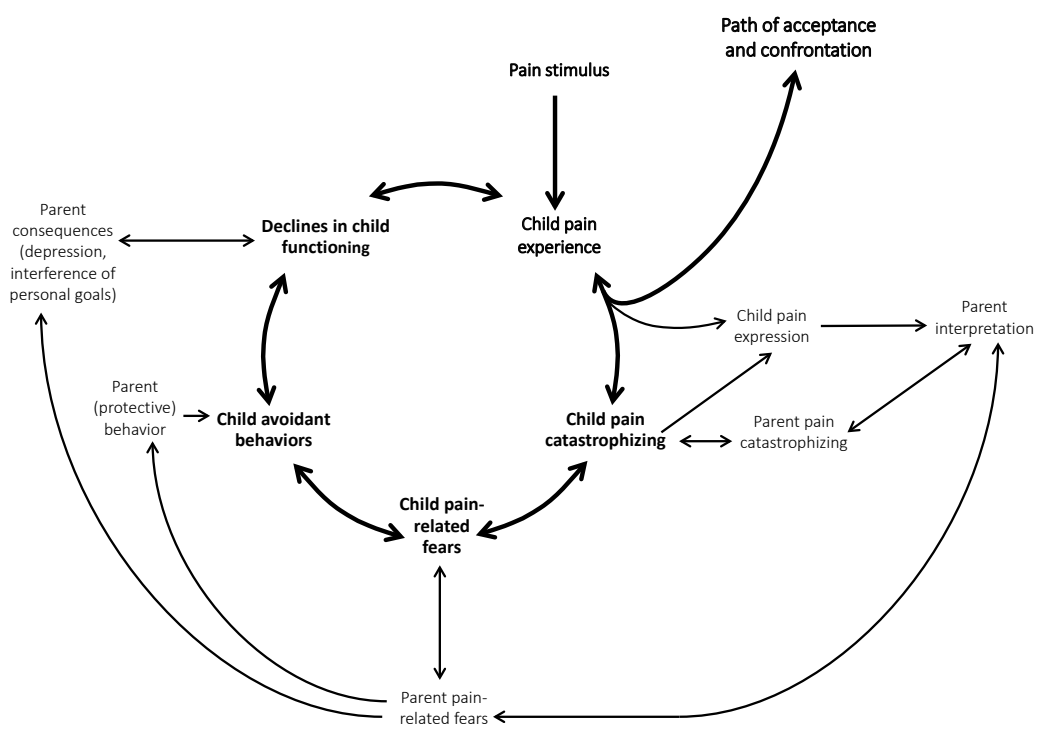

Figure 1 Note. The Interpersonal Fear Avoidance model (IFAM). In the IFAM model, a child or adolescent develops pain, often in response to an event, although it can also begin spontaneously. In the context of the child's pain experience, they either respond with progressive confrontation and acceptance of pain in their life for now or continue to perceive the presence of pain as threatening. This expression of threat and fear is observed and interpreted by the parent and reciprocally interacts with a parent's own catastrophic thinking about their child pain. This in turn leads to hypervigilance and persistent fear in the child and parent. In the context of heightened fear, the child avoids activities perceived as potentially harmful to him/her with parent protective behavior providing further encouragement for avoidance behavior. Ultimately, this lead to declines in child functioning and limitations in the life of the parent/caregiver.

Adapted from Simons et al, 2015 PAIN, with permission. 
Since pain-related fear was demonstrated to be present in adolescents and the underlying theoretical model on which graded exposure in vivo is based was shown to be applicable to adolescents, it was hypothesized that graded exposure in vivo could also work for adolescents to decrease functional disability. Specifically for adolescents with chronic musculoskeletal pain, an interdisciplinary graded exposure program was developed to be offered in outpatient specialized rehabilitation care. The exposure program consists of an adolescent module and a module for parents. The exposure program was designed as an individual treatment for adolescents, whereas the module for parents was intended to be offered in a group setting. The interdisciplinary treatment team offering the program was led by a consultant in rehabilitation medicine, and further consisted of a psychologist, and a physiotherapist or occupational therapist.

The parent module of the exposure program was developed to involve the family system as part of the treatment of the adolescent (60). These family interactions are also accentuated in the underlying theoretical model IFAM. At the moment of development of this module for parents, effective methods of intervening with parents, for example as part of an adolescent-focused treatment had not been developed (61). However, Palermo \& Eccleston (61) state that parents could benefit from the provision of education and support regarding their own adjustment and response to their adolescent in pain, and from specific information on how they can support their child. These topics have been incorporated in the parent module. The parent module was designed to help parents to provide a context in which their adolescent can change, with the ultimate goal of improving adolescent outcomes (60). It has been demonstrated that participating in the parent module was feasible in the daily life of the parents (60). Effects of the exposure program on the parents of adolescents participating in our study will not be part of this dissertation.

In addition, the exposure program for adolescents also includes a physical training for adolescents who have a diagnosis of joint hypermobility syndrome. Prevalence rates of $40 \%-55 \%$ of joint hypermobility in youth with musculoskeletal pain visiting specialized care, far exceed prevalence rates of around $27 \%$ in the general population (62-64). Joint hypermobility is characterized by generalized collagen laxity and increased joint mobility (65). When joint hypermobility is associated with musculoskeletal complaints such as pain but without and underlying tissue disease, it is referred to as (benign) joint hypermobility syndrome $(63,66)$. Symptoms in joint hypermobility syndrome can lead to functional impairments as decreased joint position sense, impaired proprioception, decreased strength and impaired balance and gait (67-70). Besides these physical consequences, consequences of joint hypermobility syndrome give rise to clinical consequences beyond impairments based on collagen laxity only. It has been demonstrated that children and adolescents with joint hypermobility have increased levels of anxiety and depression $(71,72)$. As a result, multidisciplinary treatment approaches in adolescents with both chronic pain and joint hypermobility syndrome have been recommended (73). For this reason, physical training was added to the interdisciplinary exposure program, exclusively for adolescents who were diagnosed with joint hypermobility syndrome. The physical training aims to increase muscular compensation for hypermobility, so that negative consequences of hypermobility do not hinder adolescents to become more physically active (73), during the exposure sessions. 


\section{Challenges in measuring pain-related fear and related constructs in adolescent chronic pain}

In recent literature, pain-related fear has received growing attention as being a very important construct in relation to the development and maintenance of chronic pain complaints in adults and adolescents. Additionally, pain-related fear has been identified as an important factor to focus treatment upon in order to decrease disability in adults, suggesting this approach could also be beneficial in adolescents. Measurement instruments can be used to identify the presence of pain-related fear. Recently, a systematic review and meta-analysis on assessment of pain anxiety, pain catastrophizing and fear of pain in adolescents has been published (36). Three measures were identified that assessed fear of pain, the Fear of Pain Questionnaire for children (74), the Pediatric Pain Fear Questionnaire (75) and the Photograph Series of Daily Activities (PHODA-Youth) (76).

In pediatric chronic pain, recommendations have been made to promote standardization of domains and measures in clinical trials in pediatric pain, to assist in comparison and data pooling ultimately to promote evidence-based treatment (77). An important prerequisite to attain these goals is for measures to be used in adolescent with chronic pain in different international populations. Valid translation and evaluation of psychometric properties in diverse adolescent chronic pain populations is necessary. Since no measurement instrument for assessing pain-related fear and avoidance behavior existed in Dutch at the onset of our project, the Fear of Pain Questionnaire was translated into Dutch and psychometric properties were investigated. The Fear of Pain Questionnaire was chosen because of good results in the original validation.

\section{This dissertation}

The primary focus of this dissertation is on specialized rehabilitation treatment for adolescents with chronic musculoskeletal pain. The main aim of this dissertation is to add to the scientific evidence on rehabilitation treatment for adolescents with chronic musculoskeletal pain, with the ultimate goal to ameliorate negative consequences of their complaints and increase their quality of life.

Evidence on treatment effectiveness in adults with chronic musculoskeletal pain, evidence on the negative consequences of pain-related fear in adolescents and theoretical advances in the mechanism explaining the development and maintenance of chronic pain in adolescence have led to the development of an interdisciplinary graded exposure program for adolescents and parents. This exposure program was offered in specialized rehabilitation care. A randomized clinical trial was designed to evaluate the effectiveness of this exposure program compared with usual care in four rehabilitation centers in the Netherlands (Chapter 2). The results of this trial are presented in Chapter 3. Further, we wanted to verify whether the new treatment was actually carried out according to the program protocol, and to evaluate the feasibility of working with the program in rehabilitation care. Therefore, results on feasibility and protocol adherence by treatment teams offering the exposure program are presented in Chapter 4. Additionally, one of the key 
elements of chronic pain, that is targeted in the exposure program, is pain-related fear. Chapter 5 reports on the translation into Dutch language, and validation of psychometric properties of a measure for fear of pain and activity avoidance, the Fear of Pain Questionnaire. Finally, a general discussion on the main findings, conclusions, and recommendations are presented in Chapter 6. 


\section{REFERENCES}

1. Merskey H, Bogduk N. Classification of Chronic pain. Second ed. Seattle: IASP Press; 1994.

2. Goubert L, Simons LE. Cognitive styles and processes in paediatric pain. In: McGrath PA, Stevens BJ, Walker SM, Zempsky WT, editors. Oxford Textbook of Paediatric Pain. Oxford: Oxford University Press; 2014. p. 95-101.

3. Goubert L, Craig KD, Vervoort T, Morley S, Sullivan MJ, de CWAC, et al. Facing others in pain: the effects of empathy. Pain. 2005;118(3):285-8.

4. Jordan A. Parenting an Adolescent with Chronic Pain: Impact on Parents and Association with Adolescent Functioning. Rev Pain. 2010;4(1):13-7.

5. Palermo TM, Valrie CR, Karlson CW. Family and parent influences on pediatric chronic pain: a developmental perspective. Am Psychol. 2014;69(2):142-52.

6. Vervoort T, Logan DE, Goubert L, De Clercq B, Hublet A. Severity of pediatric pain in relation to school-related functioning and teacher support: an epidemiological study among school-aged children and adolescents. Pain. 2014;155(6):1118-27.

7. Logan DE, Simons LE, Stein MJ, Chastain L. School impairment in adolescents with chronic pain. J Pain. 2008;9(5):407-16.

8. Finley GA, MacLaren Chorney J, Campbell L. Not small adults: the emerging role of pediatric pain services. Can J Anaesth. 2014;61 (2):180-7.

9. Perquin CW, Hazebroek-Kampschreur AAJM, Hunfeld JAM, Bohnen AM, van Suijlekom-Smit LWA, Passchier J, et al. Pain in children and adolescents: a common experience. Pain. 2000;87(1):51-8.

10. Eccleston C, Bruce E, Carter B. Chronic pain in children and adolescents. Paediatr Nurs. 2006;18(10):30-3.

11. King S, Chambers CT, Huguet A, MacNevin RC, McGrath PJ, Parker L, et al. The epidemiology of chronic pain in children and adolescents revisited: A systematic review. Pain. 2011;152(12):2729-38.

12. Palermo T, Eccleston C, Goldschneider K, Larkin K, Sethna N, Schechter N, et al. Assessment and Management of Chidlren with Chronic Pain A position statement from the American Pain Society. American Pain Society. 2012.

13. Roth-Isigkeit A, Thyen U, Stoven H, Schwarzenberger J, Schmucker P. Pain among children and adolescents: restrictions in daily living and triggering factors. Pediatrics. 2005;115(2):e152-62.

14. Huguet A, Miro J. The severity of chronic pediatric pain: an epidemiological study. J Pain. 2008;9(3):22636.

15. El-Metwally A, Salminen JJ, Auvinen A, Kautiainen H, Mikkelsson M. Prognosis of non-specific musculoskeletal pain in preadolescents: a prospective 4-year follow-up study till adolescence. Pain. 2004;110(3):550-9.

16. Mikkelsson M, El-Metwally A, Kautiainen H, Auvinen A, Macfarlane GJ, Salminen JJ. Onset, prognosis and risk factors for widespread pain in schoolchildren: a prospective 4-year follow-up study. Pain. 2008;138(3):681-7.

17. Fearon P, Hotopf M. Relation between headache in childhood and physical and psychiatric symptoms in adulthood: national birth cohort study. BMJ. 2001;322(7295):1145.

18. Walker LS, Dengler-Crish CM, Rippel S, Bruehl S. Functional abdominal pain in childhood and adolescence increases risk for chronic pain in adulthood. Pain. 2010;150(3):568-72. 
19. Palermo TM, Eccleston C, Lewandowski AS, Williams ACdC, Morley S. Randomized controlled trials of psychological therapies for management of chronic pain in children and adolescents: An updated meta-analytic review. Pain. 2010;148(3):387-97.

20. Hechler T, Kanstrup M, Holley AL, Simons LE, Wicksell R, Hirschfeld G, et al. Systematic Review on Intensive Interdisciplinary Pain Treatment of Children With Chronic Pain. Pediatrics. 2015;136(1):115-27.

21. Hunfeld JA, Perquin CW, Duivenvoorden HJ, Hazebroek-Kampschreur AA, Passchier J, van Suijlekom-Smit LW, et al. Chronic pain and its impact on quality of life in adolescents and their families. J Pediatr Psychol. 2001;26(3):145-53.

22. Simons LE, Basch MC. State of the art in biobehavioral approaches to the management of chronic pain in childhood. Pain Manag. 2015.

23. Gatchel RJ, Peng YB, Peters ML, Fuchs PN, Turk DC. The biopsychosocial approach to chronic pain: scientific advances and future directions. Psychol Bull. 2007;133(4):581-624.

24. Claar RL, Walker LS. Functional assessment of pediatric pain patients: psychometric properties of the functional disability inventory. Pain. 2006;121(1-2):77-84.

25. Eccleston C, Crombez G, Scotford A, Clinch J, Connell H. Adolescent chronic pain: patterns and predictors of emotional distress in adolescents with chronic pain and their parents. Pain. 2004;108(3):221-9.

26. Peterson CC, Palermo TM. Parental Reinforcement of Recurrent Pain: The Moderating Impact of Child Depression and Anxiety on Functional Disability. J Pediatr Psychol. 2004;29(5):331-41.

27. Von Korff M, Dworkin SF, Le Resche L. Graded chronic pain status: an epidemiologic evaluation. Pain. 1990;40(3):279-91.

28. Connelly M, Rapoff MA. Assessing health-related quality of life in children with recurrent headache: reliability and validity of the PedsQLTM 4.0 in a pediatric headache sample. J Pediatr Psychol. 2006;31(7):698-702.

29. Konijnenberg AY, Uiterwaal CS, Kimpen JL, van der Hoeven J, Buitelaar JK, de Graeff-Meeder ER. Children with unexplained chronic pain: substantial impairment in everyday life. Arch Dis Child. 2005;90(7):680-6.

30. Gauntlett-Gilbert J, Eccleston C. Disability in adolescents with chronic pain: Patterns and predictors across different domains of functioning. Pain. 2007;131(1-2):132-41.

31. Kashikar-Zuck S, Goldschneider KR, Powers SW, Vaught MH, Hershey AD. Depression and functional disability in chronic pediatric pain. Clin J Pain. 2001;17(4):341-9.

32. Cohen LL, Vowles KE, Eccleston C. The impact of adolescent chronic pain on functioning: disentangling the complex role of anxiety. The Journal of Pain. 2010;11(11):1039-46.

33. Caes L, Fisher E, Clinch J, Tobias JH, Eccleston C. The role of pain-related anxiety in adolescents' disability and social impairment: ALSPAC data. Eur J Pain. 2015;19(6):842-51.

34. Simons LE. Fear of pain in children and adolescents with neuropathic pain and complex regional pain syndrome. Pain. 2016;157 Suppl 1:S90-7.

35. Simons LE, Kaczynski KJ, Conroy C, Logan DE. Fear of pain in the context of intensive pain rehabilitation among children and adolescents with neuropathic pain: associations with treatment response. The Journal of Pain. 2012;13(12):1151-61.

36. Fisher E, Heathcote LC, Eccleston C, Simons LE, Palermo TM. Assessment of Pain Anxiety, Pain Catastrophizing, and Fear of Pain in Children and Adolescents With Chronic Pain: A Systematic Review and Meta-Analysis. J Pediatr Psychol. 2018;43(3):314-25. 
37. Martin AL, McGrath PA, Brown SC, Katz J. Anxiety sensitivity, fear of pain and pain-related disability in children and adolescents with chronic pain. Pain research \& management. 2007;12(4):267-72.

38. Palermo TM. Impact of recurrent and chronic pain on child and family daily functioning: a critical review of the literature. J Dev Behav Pediatr. 2000;21(1):58-69.

39. Sleed M, Eccleston C, Beecham J, Knapp M, Jordan A. The economic impact of chronic pain in adolescence: methodological considerations and a preliminary costs-of-illness study. Pain. 2005;119(13):183-90.

40. Lewandowski AS, Palermo TM, Stinson J, Handley S, Chambers CT. Systematic review of family functioning in families of children and adolescents with chronic pain. J Pain. 2010;11(11):1027-38.

41. Claar RL, Simons LE, Logan DE. Parental response to children's pain: the moderating impact of children's emotional distress on symptoms and disability. Pain. 2008;138(1):172-9.

42. Palermo TM, Chambers CT. Parent and family factors in pediatric chronic pain and disability: an integrative approach. Pain. 2005;119(1-3):1-4.

43. Lynch-Jordan AM, Kashikar-Zuck S, Szabova A, Goldschneider KR. The interplay of parent and adolescent catastrophizing and its impact on adolescents' pain, functioning, and pain behavior. Clin J Pain. 2013;29(8):681-8.

44. Caes L, Vervoort T, Eccleston C, Vandenhende M, Goubert L. Parental catastrophizing about child's pain and its relationship with activity restriction: the mediating role of parental distress. Pain. $2011 ; 152(1): 212-22$.

45. Groenewald CB, Essner BS, Wright D, Fesinmeyer MD, Palermo TM. The economic costs of chronic pain among a cohort of treatment-seeking adolescents in the United States. J Pain. 2014;15(9):925-33.

46. Groenewald CB, Palermo TM. The price of pain: the economics of chronic adolescent pain. Pain Manag. 2015;5(2):61-4.

47. Perquin CW, Hunfeld JA, Hazebroek-Kampschreur AA, van Suijlekom-Smit LW, Passchier J, Koes BW, et al. Insights in the use of health care services in chronic benign pain in childhood and adolescence. Pain. 2001;94(2):205-13.

48. Meerding WJ, Bonneux L, Polder JJ, Koopmanschap MA, van der Maas PJ. Demographic and epidemiological determinants of healthcare costs in Netherlands: cost of illness study. BMJ. 1998;317(7151):1115.

49. Maniadakis N, Gray A. The economic burden of back pain in the UK. Pain. 2000;84(1):95-103.

50. Kashikar-Zuck S. Treatment of children with unexplained chronic pain. Lancet. 2006;367(9508):380-2.

51. Landry BW, Fischer PR, Driscoll SW, Koch KM, Harbeck-Weber C, Mack KJ, et al. Managing Chronic Pain in Children and Adolescents: A Clinical Review. PM \& R : the journal of injury, function, and rehabilitation. 2015;7(11 Suppl):S295-315.

52. Vlaeyen JW, Linton SJ. Fear-avoidance and its consequences in chronic musculoskeletal pain: a state of the art. Pain. 2000;85(3):317-32.

53. Verbunt JA, Seelen HA, Vlaeyen JW, van der Heijden GJ, Knottnerus JA. Fear of injury and physical deconditioning in patients with chronic low back pain. Arch Phys Med Rehabil. 2003;84(8):1227-32.

54. Turk DC, Wilson HD. Fear of pain as a prognostic factor in chronic pain: conceptual models, assessment, and treatment implications. Current pain and headache reports. 2010;14(2):88-95.

55. Simons LE, Kaczynski KJ. The Fear Avoidance model of chronic pain: examination for pediatric application. The Journal of Pain 2012;13(9):827-35. 
56. Vlaeyen JW, de Jong J, Geilen M, Heuts PH, van Breukelen G. The treatment of fear of movement/(re) injury in chronic low back pain: further evidence on the effectiveness of exposure in vivo. Clin J Pain. 2002;18(4):251-61.

57. de Jong JR, Vlaeyen JW, Onghena P, Cuypers C, den Hollander M, Ruijgrok J. Reduction of pain-related fear in complex regional pain syndrome type I: the application of graded exposure in vivo. Pain. 2005; 116(3):264-75.

58. Leeuw M, Goossens ME, van Breukelen GJ, de Jong JR, Heuts PH, Smeets RJ, et al. Exposure in vivo versus operant graded activity in chronic low back pain patients: results of a randomized controlled trial. Pain. 2008;138(1):192-207.

59. den Hollander M, Goossens M, de Jong J, Ruijgrok J, Oosterhof J, Onghena P, et al. Expose or protect? A randomized controlled trial of exposure in vivo vs pain-contingent treatment as usual in patients with complex regional pain syndrome type 1. Pain. 2016;157(10):2318-29.

60. Wiertz C, Goossens M, Spek EM, Verbunt JA. A cognitive-behavioral program for parents of children with chronic musculoskeletal pain; A feasibility study. Eur J Pain. 2017;21(9):1571-81.

61. Palermo TM, Eccleston C. Parents of children and adolescents with chronic pain. Pain. 2009;146(12):15-7.

62. de Inocencio J. Musculoskeletal pain in primary pediatric care: analysis of 1000 consecutive general pediatric clinic visits. Pediatrics. 1998;102(6):E63.

63. Gedalia A, Press J, Klein M, Buskila D. Joint hypermobility and fibromyalgia in schoolchildren. Ann Rheum Dis. 1993;52(7):494-6.

64. Clinch J, Deere K, Sayers A, Palmer S, Riddoch C, Tobias JH, et al. Epidemiology of generalized joint laxity (hypermobility) in fourteen-year-old children from the UK: a population-based evaluation. Arthritis Rheum. 2011;63(9):2819-27.

65. Grahame R. Hypermobility: an important but often neglected area within rheumatology. Nat Clin Pract Rheumatol. 2008;4(10):522-4.

66. Wolf JM, Cameron KL, Owens BD. Impact of joint laxity and hypermobility on the musculoskeletal system. J Am Acad Orthop Surg. 2011;19(8):463-71.

67. Sahin N, Baskent A, Cakmak A, Salli A, Ugurlu H, Berker E. Evaluation of knee proprioception and effects of proprioception exercise in patients with benign joint hypermobility syndrome. Rheumatol Int. 2008;28(10):995-1000.

68. Rombaut L, De Paepe A, Malfait F, Cools A, Calders P. Joint position sense and vibratory perception sense in patients with Ehlers-Danlos syndrome type III (hypermobility type). Clin Rheumatol. 2010;29(3):289-95.

69. Hanewinkel-van Kleef YB, Helders PJ, Takken T, Engelbert RH. Motor performance in children with generalized hypermobility: the influence of muscle strength and exercise capacity. Pediatr Phys Ther. 2009;21(2):194-200.

70. Rombaut L, Malfait F, De Wandele I, Thijs Y, Palmans T, De Paepe A, et al. Balance, gait, falls, and fear of falling in women with the hypermobility type of Ehlers-Danlos syndrome. Arthritis Care Res (Hoboken). 2011;63(10):1432-9.

71. Garcia-Campayo J, Asso E, Alda M. Joint Hypermobility and Anxiety: The State of the Art. Current Psychiatry Reports. 2011;13(1):18-25. 
72. Smith TO, Easton V, Bacon H, Jerman E, Armon K, Poland F, et al. The relationship between benign joint hypermobility syndrome and psychological distress: a systematic review and meta-analyses. Rheumatology (Oxford). 2013.

73. Scheper MC, Engelbert RH, Rameckers EA, Verbunt J, Remvig L, Juul-Kristensen B. Children with generalised joint hypermobility and musculoskeletal complaints: state of the art on diagnostics, clinical characteristics, and treatment. Biomed Res Int. 2013;2013:121054.

74. Simons LE, Sieberg CB, Carpino E, Logan D, Berde C. The Fear of Pain Questionnaire (FOPQ): Assessment of Pain-Related Fear Among Children and Adolescents With Chronic Pain. The Journal of Pain. 2011;12(6):677-86.

75. Huguet A, McGrath PJ, Pardos J. Development and preliminary testing of a scale to assess pain-related fear in children and adolescents. The Journal of Pain 2011;12(8):840-8.

76. Verbunt JA, Nijhuis A, Vikstrom M, Stevens A, Haga N, de Jong J, et al. The psychometric characteristics of an assessment instrument for perceived harmfulness in adolescents with musculoskeletal pain (PHODA-youth). Eur J Pain. 2015;19(5):695-705.

77. McGrath PJ, Walco GA, Turk DC, Dworkin RH, Brown MT, Davidson K, et al. Core outcome domains and measures for pediatric acute and chronic/recurrent pain clinical trials: PedIMMPACT recommendations. J Pain. 2008;9(9):771-83. 



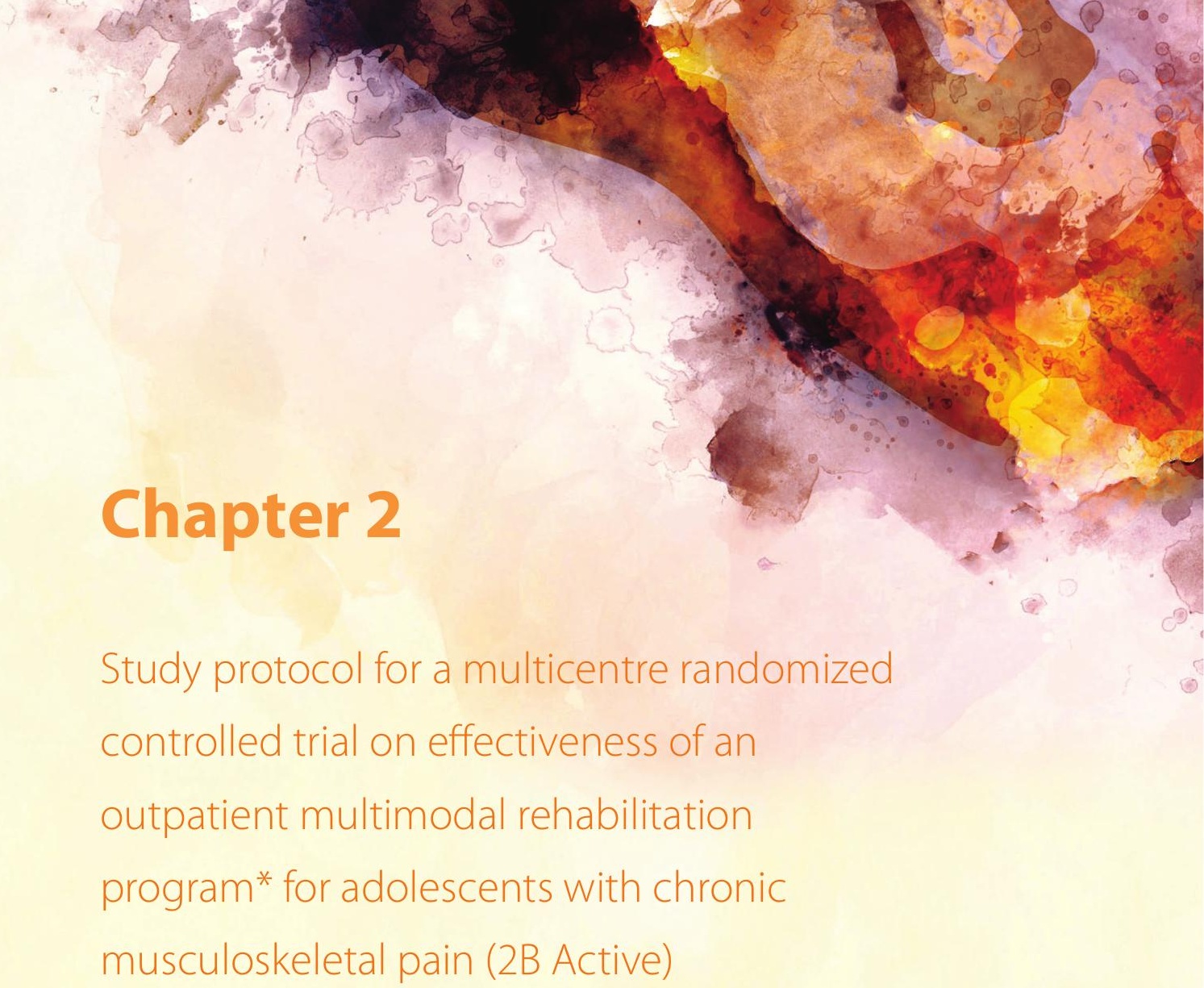

Carolien Dekker

Mariëlle Goossens

Caroline Bastiaenen

Jeanine Verbunt

BMC Musculoskeletal Disorders (2016) DOI 10.1186/s12891-016-1178-5

* In December 2017 IASP published new definitions on their website, that made us change the name of our intervention. Hereafter, the multimodal rehabilitation program is called an interdisciplinary graded exposure program. 


\section{ABSTRACT}

Background Chronic musculoskeletal pain (CMP) in adolescents can influence functioning and well-being, and has negative consequences for families and society as well. According to the Fear Avoidance Model, fear of movement and pain catastrophizing can influence the occurrence and maintenance of chronic pain complaints and functional disability. Primary objective is to evaluate the effectiveness of a multimodal rehabilitation program in reducing functional disability for adolescents with CMP compared with care as usual.

Methods/Design Pragmatic multicentre parallel group randomized controlled trial. Randomization by minimization (ratio 1:1) and treatment allocation will be concealed, computer-generated and performed by an independent organization. After randomization, data collection and researchers remain blinded. Inclusion of 124 adolescents and their parents is intended. This sample size is based on a $25 \%$ difference in group mean on the primary outcome, with $a=5 \%, \beta=80 \%$ and expected $15 \%$ loss to follow up. Study population are adolescents (12-21 years) with CMP with an indication for outpatient rehabilitation treatment in the Netherlands.

The intervention group receives a Multimodal Rehabilitation Program (MRP), a multidisciplinary outpatient individual rehabilitation program. MRP consists of 2 different treatment approaches: A graded exposure module or a combination module of graded exposure and physical training. Selection a module depends on the needs of the patient. To both modules a parent module is added. The control group receives care as usual, which is the care currently provided in Dutch rehabilitation centres. Treatment duration varies between 7 and 16 weeks, depending on treatment allocation.

Self-reported measurements are at baseline, and at 2, 4, 10 and 12 months after start of treatment. Intention to treat analysis for between group differences on all outcome variables will be performed. Primary outcome is functional disability (Functional Disability Inventory). Secondary outcome variables are fear of pain, catastrophizing, perceived harmfulness, pain intensity, depressive symptoms, and quality of life. Total direct and indirect costs and health related quality of life will be measured. Process evaluation focuses on protocol adherence, patient centeredness and treatment expectations.

Discussion A pragmatic approach was chosen, to ensure that results obtained are most applicable to daily practice.

Trial registration Clinicaltrials.gov ID: NCT02181725 (7 February 2014)

Funded by Fonds Nuts Ohra, Stichting Vooruit, and Adelante

\section{Keywords}

Chronic pain, Adolescent, Graded Exposure Therapy, RCT, Pragmatic, Economic evaluation, Rehabilitation 


\section{BACKGROUND}

Chronic pain in children and adolescents is a major health concern in the paediatric population (1-3). Up to $25 \%$ of Dutch schoolchildren, especially adolescents, report pain for 3 months or longer (=chronic pain) $(1,4)$. Internationally, prevalence rates for musculoskeletal pain vary between 11 and 38\% (3). Chronic musculoskeletal pain is, together with headache and abdominal pain, one of the most reported pain complaints in adolescents $(1,5)$. Although a broad array of medical diagnoses is involved in adolescent pain conditions, in only 10-30\% of cases a specific medical disease explaining the pain is identified $(6,7)$. Unexplained musculoskeletal pain is not self-limiting: persistence rates of pain up to $30-64 \%$ after 4 years have been reported $(5,8,9)$. Pain during adolescence even increases the risk of having a chronic pain syndrome in adulthood $(5,10)$.

\section{The consequences of musculoskeletal pain}

Most adolescents function quite well regardless of pain. Nonetheless, in approximately $40 \%$ of the adolescents with chronic pain, pain has a disabling impact on daily functioning (11-13). Pain can interfere with developmental, school and leisure time activities, and causes serious psychological distress. It also has an impact on the adolescent's families. Primary caregivers report more restrictions in social life and more problems with coping with the adolescent's pain (14-16). In addition to the psychological burden, there is little known about the financial burden of caring for a child with chronic pain (17-19).

Although the body of evidence on adult chronic pain and different rehabilitation treatments is growing, research into adolescents who suffer from chronic pain complaints is still in its infancy. Rehabilitation care for adolescents with chronic pain differs from adult care in several ways. First, in adolescent chronic pain rehabilitation, the family system, and especially influences of the parents are important to take into account. Parental behaviour, such as protective behaviour, has been shown to influence adolescent's response to pain. This can result in greater functional disability, higher school absence and more depressive symptoms in the children (15, 20-25). Second, situations and activities that contribute to developing and maintaining adolescent chronic pain are not completely similar to those contributing to adult chronic pain $(26,27)$. Third, in adolescents with chronic pain, prevalence rates of hypermobility (40-55\%) are higher than in healthy adolescents. Hypermobility as such is not a problem, but when hypermobility is associated with complaints, such as pain, it is called joint hypermobility syndrome (HMS) $(28,29)$ thereby making HMS a contributing factor in treating adolescent chronic pain (30-33).

\section{Theoretical framework}

According to the fear avoidance model of chronic pain (34), both fear of pain/movement and catastrophic thinking about pain can lead to the development and maintenance of chronic pain problems. Simons and Kaczynski (35) studied the applicability of the fear avoidance model in the paediatric population and concluded that the model is applicable, after making some modi- 
fications to account for developmental aspects at child/adolescent age. Goubert and Simons (25) presented an interpersonal fear avoidance model of pain, in which the social context of the adolescent becomes clear.

The implications of these models for a treatment approach of adolescent CMP is that lowering fear of pain/movement and accompanying catastrophic thinking could lower avoidance behaviour and increase functional ability (34). Moreover, the interpersonal fear avoidance model accentuates the necessity to involve the family system as part of the treatment, in a way that a context can be created in which the adolescent can change. Adding the parental influences to the fear avoidance model is an important difference between the model for adults and the model for adolescents.

\section{Treatment of chronic pain}

Adolescent chronic pain is regarded a complex health problem that requires a multidisciplinary treatment approach from a biopsychosocial perspective $(17,36,37)$. This study focuses on multidisciplinary outpatient treatment for adolescents with CMP. Outpatient treatment currently provided to adolescents in the Netherlands is mostly based on the principles of Graded Activity (GA). There is a need for more RCT's that investigate effectiveness of different patient (and parent) interventions in paediatric CMP $(17,22,38)$. The MRP that is investigated in this study is based on Graded Exposure therapy (GE). GE specifically aims at challenging catastrophizing thoughts and performing feared activities in order to improve functional ability of patients with chronic pain. In adults with chronic pain this treatment approach is successful $(39,40)$. The Maastricht University Medical Centre (MUMC) has recently developed a multimodal rehabilitation program (MRP), aimed specifically at improving adolescent's functional ability by reducing pain-related fear. The Multimodal Rehabilitation Program consists of 2 different treatment approaches: A graded exposure module or a combination module of graded exposure and physical training. Both approaches also include a parent module. The choice of graded exposure module or combination module depends on the needs of the patient (Table1).

\section{Aims of the study}

This study investigates the effectiveness of a multimodal rehabilitation program (MRP) including Graded Exposure treatment in reducing functional disability (measured with the Functional Disability Inventory on short and long term) in adolescents (12-21 years) with chronic musculoskeletal pain compared with care as usual (CAU).

Secondary aims are a) to compare the cost-effectiveness and cost-utility of both interventions, also during the follow-up period, an b) to evaluate the feasibility of working with MRP in terms of treatment fidelity and patient centeredness. 


\section{METHODS}

\section{Study design and participants}

A two group pragmatic randomized controlled trial is undertaken with adolescents with CMP, with follow-up assessment at 2, 4, 10 and 12 months after start of treatment. Adolescents will be allocated to either intervention (MRP) or control (CAU) with a ratio 1:1. Patients who receive an indication for outpatient rehabilitation treatment will be invited to participate and will be randomized to either usual care (CAU) or the intervention (MRP) after informed consent is obtained. Parents will be asked to participate as well.

Estimates for the calculation of the sample size are based on the primary outcome measure, the Functional Disability Inventory (FDI). An average FDI total score of 23 (average score obtained from adolescents that were treated at the Maastricht University Medical Centre in the year before the start of this study), a standard deviation of 9.2 and an expected mean difference between intervention and control condition of 5 points on the total FDI-score were used. A difference of 5 points equals approximately a 25\% difference in mean FDI scores between intervention and control group.

Given $a=0.05$, two sided testing, a power of $80 \%$, and anticipating $15 \%$ loss to follow-up, a sample size of 62 participants per trial-arm was calculated. For two trial arms, this results in a total sample size of $\mathrm{N}=124$.

Adolescents will be recruited from 4 centres in The Netherlands: Maastricht University Medical Centre/Adelante, Laurentius Hospital Roermond, Revant Rehabilitation centre Breda and Rijndam Rehabilitation Centre in Rotterdam. Both treatments offered are embedded in the daily care process in each treatment centre, with an inclusion period of 1.5 years. Ethical approval for this trial was granted by the Medical Ethics Committee Academic Hospital Maastricht/Maastricht University, the Netherlands, NL47323.068.13/METC13-3-062

\section{Eligibility criteria}

Adolescents with a treatment indication for outpatient rehabilitation care for treatment of CMP, aged between 12-21 years and with adequate Dutch literacy were eligible for inclusion. Patients will be excluded if there is a) any suspicion of a medical (orthopaedic, rheumatic or neurological) disease, that can fully explain the current level of severity of pain complaints, b) any suspicion of an (underlying) psychiatric disease that hampers rehabilitation treatment or c) pregnancy.

\section{Randomization and allocation concealment}

Patients will be randomized by minimization after informed consent is obtained. Minimization (41) was chosen to balance treatment centre, sex and age factors within the study. To execute the randomization procedures a validated electronic randomization system (ALEA, offered by the 
Clinical Trial Centre Maastricht, (TCM) will be used. Randomization and treatment allocation are completely independent of the study and blinded for participants including parents and all researchers, including raters, and at that moment for caregivers and the consultant in rehabilitation medicine. After treatment allocation is revealed, blinding cannot be maintained for care givers. Adolescents and parents will be kept naïve concerning the comparison between the interventions. The research team remains blinded during the course of the study. Data collection is also blinded.

\section{Interventions}

Contrast between the interventions is found in their theoretical basis and point of engagement where the treatment focuses on. Graded Exposure uses principles from classical conditioning and cognitive therapeutic techniques to systematically reduce pain-related fear and catastrophic thinking in adolescents with chronic pain (42). Graded Activity uses principles of operant conditioning, resulting in rewarding healthy and age adequate daily functional activities performed by the adolescent and a stepwise increase of the adolescent's activity levels (42).

Parents are present and are invited to participate in both the intervention and control condition. However, in the intervention condition (MRP), parents receive additional 3 group meetings (the parent module) with the treatment team (Table 1). Meaning that in the MRP parents are involved in the treatment of their adolescent in two ways. They are both present and participate during treatment of their child, but additionally they participate in a separate parent module.

Both treatments are provided by a multidisciplinary treatment team, including a consultant in rehabilitation medicine, a physiotherapist or occupational therapist and a psychologist/behavioural therapist.

\section{Intervention group}

In table1 the elements of the three modules of the MRP are described. Graded Exposure aims to increase healthy behaviour by systematically and gradually exposing adolescents to fear provoking activities and movements. Starting point for this approach is a personal hierarchy of activities feared by the adolescent. The Photograph series of Daily Activities - Youth (PHODA-Youth) (27) is used as a standardized procedure to build these personal hierarchies. All therapists in the MRP condition received training on the principles of Graded Exposure therapy and the treatment protocols, consisting of a workshop and a 3-day training provided by skilled trainers (with 3 to $>15$ year experience in Graded Exposure therapy). Administration of the PHODA-Youth was part of the 3-day training.

Adolescents in the intervention condition MRP receive a graded exposure module (7 weeks) or a combination module (15 weeks) with training and graded exposure. Their parents receive a parent module (3 meetings). Adolescents with CMP receive the graded exposure module. If an adolescent is additionally identified (Brighton criteria including Beighton score, will be used (29, 
$32,43))$ as having pain complaints related to joint hypermobility (HMS), the combination module will be offered. All parents in the MRP condition are offered the parent module, which is organised in the evening hours.

Table 1 Intervention group: Multimodal Rehabilitation Program, description of the treatment

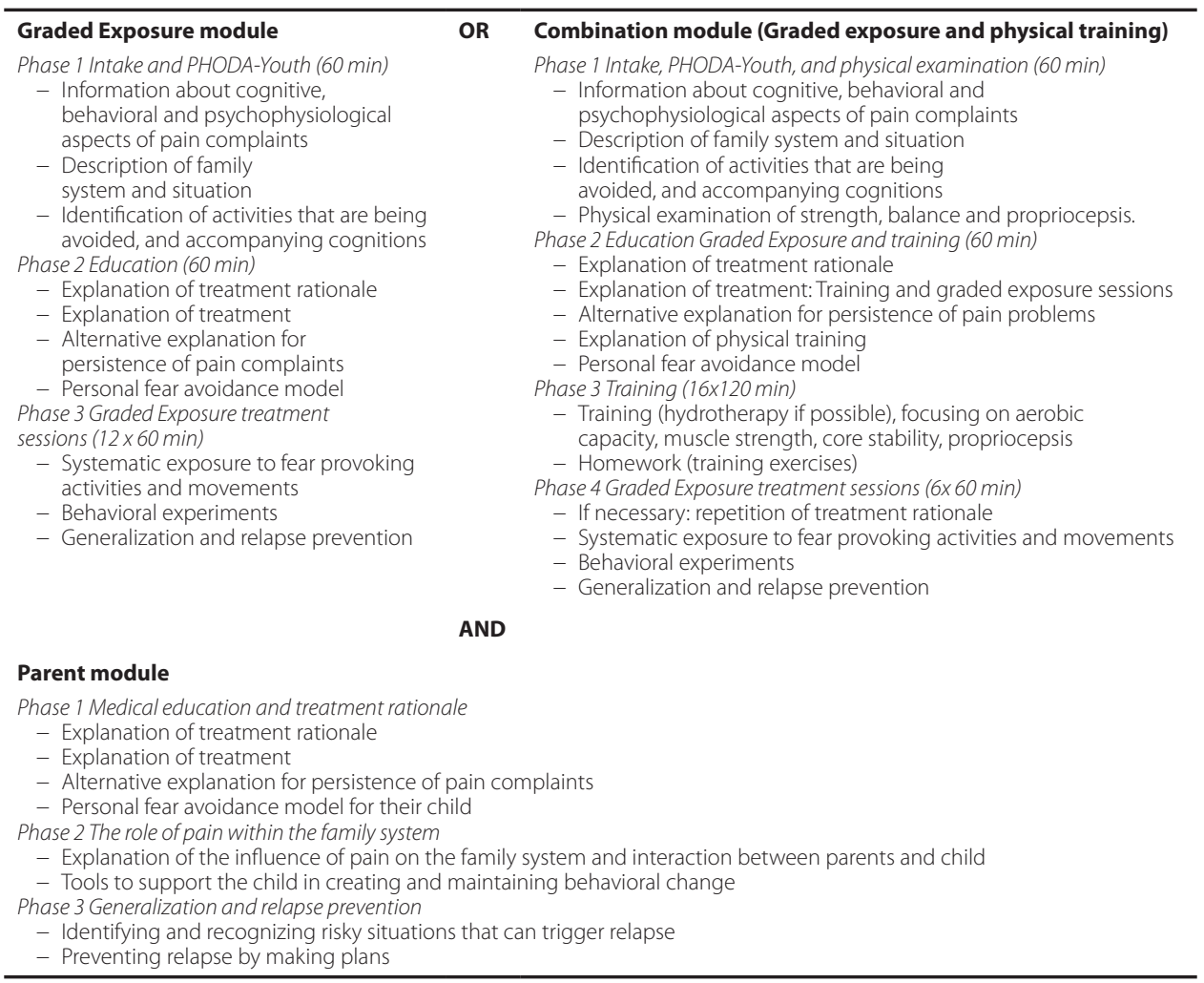




\section{Control group}

Currently, treatment of adolescents with chronic pain in a rehabilitation setting consists of multidisciplinary treatment based on the principles of Graded Activity. Table 2 presents the main treatment elements of GA. GA aims to increase healthy behaviour by employing operant learning principles such as encouraging desired behaviours (42). The treatment consists of a time contingent and stepwise increase in the level of functioning, despite complaints of pain (44). Duration of the care as usual treatment varies between 9 and 16 weeks. This variation exists because each collaborating treatment centre has developed its own specific treatment protocol, also depending on the practical and logistic possibilities of the treatment centre. Content of the treatment programmes is generally similar and principal discriminative from the content of the experimental intervention (MRP).

Table 2 Control group: Care as Usual, description of the treatment

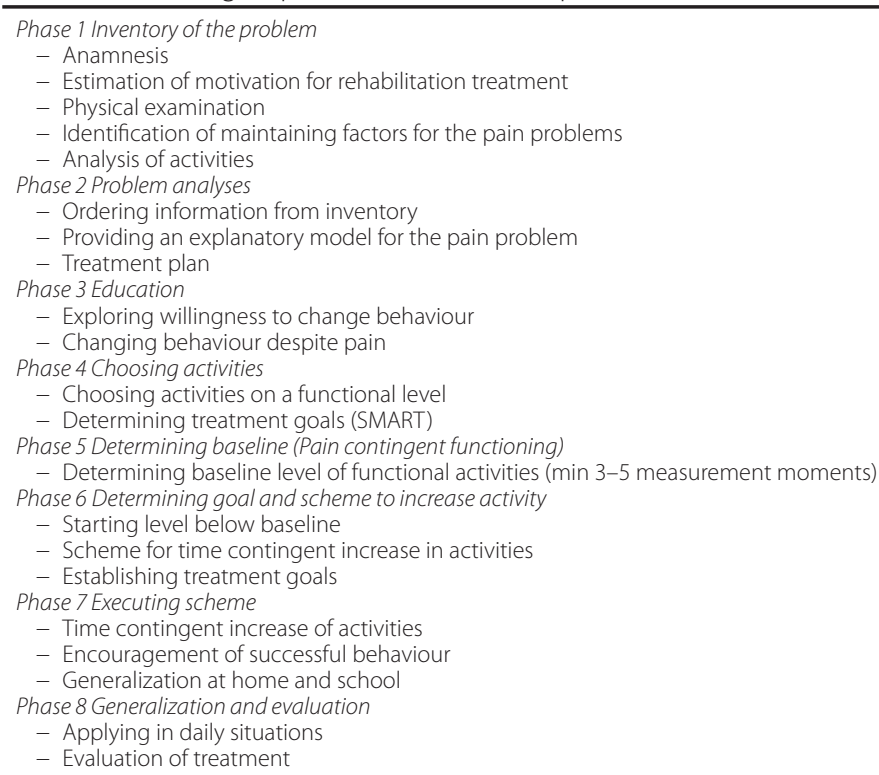




\section{Outcome measures}

PedIMMPACT recommendations were followed when choosing outcome domains and in the selection of measurement instruments for this study (45). Details of measurement instruments can be found in table 3 . Assessments will be performed at baseline (before start of treatment) and at 2, 4, 10 and 12 months after start of treatment. Primary outcome measure is adolescent's functional disability $(F D I)(46,47)$. Secondary outcome measures are adolescent fear of pain (FOPQ-C)(48), perceived harmfulness (PHODA-Youth) (27), pain catastrophizing (PCS-C) $(49,50)$, pain intensity (VAS) (51), depressive symptoms (CDI) (52) and pain specific quality of life (QLA-CP) (53), and parent perceived functional disability (FDI-P) $(46,47)$, fear of pain (FOPQ-P) $(48)$, pain catastrophizing (PCS-P) (50), adult responses to children's symptoms (ARCS) (54).

\section{Other study parameters}

Demographic variables will be measured at baseline. These variables include age, sex, educational level and school absence, family composition, ethnic background. Variables related to chronic pain that will be measured include: onset and duration of pain complaints, location of pain complaints, course of the complaints, and (if applicable) medication use.

\section{Costs measurement}

To evaluate the economic consequences, the intervention costs, other health care costs, patient and family costs, and productivity losses will be assessed. Patient and family costs include out-ofpocket costs, such as costs for informal care and extra expenses. Productivity losses (for adolescents and parents) are based on the days absent from work or school because of the pain.

At baseline, total costs will be inventoried 3 months retrospective. During the trial period (12 months) monthly cost-diaries will be used after the end of treatment. For addressing cost-effectiveness of interventions, the cost-diary method might be a successful method (55). Both the parents and the adolescents will be asked to provide information about health care utilization and school absenteeism/productivity losses. If the adolescents are 18 years or older, they may choose to fill out the cost diary themselves, below 18 years it is recommended they complete the cost diary together with their parents.

Generic health related quality of life was measured with the EQ-5D-Y (56). The EQ-5D-Y is a self-report measure of health related quality of life. QALY's can be calculated by means of the applying a tariff to obtain a weighted health state index. The adult tariff will be used for the Youth-version of the questionnaire, since no youth-tariff is available. 
Table 3 Primary and secondary outcome measures*

\begin{tabular}{lll}
\hline Primary outcome & Measurement instrument & $\begin{array}{c}\text { Time points of } \\
\text { measurement }\end{array}$ \\
\hline $\begin{array}{l}\text { Functional disability } \\
(46,47)\end{array}$ & $\begin{array}{l}\text { Functional Disability Inventory (FDI) } \\
\text { Self-report measure for perceived difficulty in performing activities } \\
\text { at school, at home and in recreational or social interactions. } \\
15 \text { Items rated on a five-point scale (0-4). Total scores range }\end{array}$ & $\begin{array}{l}\text { Baseline, } 8 \text { weeks, } \\
16 \text { weeks, } 10\end{array}$ \\
& and 12 months \\
& Valid and reliable measure for assessing pain related disability in adolescents.
\end{tabular}

\begin{tabular}{|c|c|}
\hline Secondary outcomes & Measurement instrument \\
\hline $\begin{array}{l}\text { Fear of pain } \\
\text { (48) }\end{array}$ & $\begin{array}{l}\text { Fear of Pain Questionnaire (FOPQ-C) } \\
\text { Self-report measure to assess pain-related fear in adolescents witl } \\
\text { chronic pain. } 24 \text { items rated on a five-point scale (0-4). Total score } \\
\text { range } 0-96 \text { with a higher score indicating more fear. Consists of } \\
\text { subscales 1) Fear of Pain and 2) Avoidance of activities. } \\
\text { Psychometric properties of the English version are good. }\end{array}$ \\
\hline $\begin{array}{l}\text { Perceived harmfulness } \\
\text { (27) }\end{array}$ & $\begin{array}{l}\text { PHODA-Youth } \\
\text { Measures perceived harmfulness of physical and social activities } \\
\text { and can be used to create a hierarchy of fearful activities. } \\
51 \text { photographs are rated on a scale } 0-10 \text { (steps } 0.1 \text { ). The higher } \\
\text { a photo is ranked, the more harmful the adolescent thinks } \\
\text { executing the activity is. Consists of subscales } 1 \text { ) activities of daily } \\
\text { living, 2) intensive physical activities and 3) social activities. } \\
\text { Psychometric qualities are good. }\end{array}$ \\
\hline
\end{tabular}

Time points of measurement

Baseline, 8 weeks, 16 weeks, 10 and 12 months

Baseline, 8 weeks, 16 weeks, 10 and 12 months

Pain Catastrophizing

\section{Pain Catastrophizing Scale (PCS-C)}

Self-report measure of catastrophic thinking about pain. Frequency of feelings and thoughts adolescents may experience when they are in pain are measured. 13 items rated on a five-point scale (0-4). Total scores range 0-52 with higher scores indicating more catastrophic thinking. Consists of subscales 1) Rumination, 2) Magnification, and 3) Helplessness. Reliability and validity are good.

Depressive symptoms (52)

Pain specific quality of life (53)

Pain Intensity (51)

Parent perceived Functional Disability $(46,47)$

\section{Child Depression Inventory (CDI)}

Self-report measure of depressive symptoms in children and adolescents. 27 items rated on a three-point scale (0-2). Total scores range $0-54$ with higher scores indicating more depressive symptoms. 3) Ineffectiveness, 4) Negative self-esteem, and 5) Anhedonia. Dutch version demonstrates good reliability and validity.

\section{Quality of Life in Adolescent with Chronic Pain (QLA-CP)}

Self-reported pain-specific quality of life measure

for adolescents with chronic pain

44 items in 6 domains, 1) Psychological functioning, 2) Functional status, 3) Physical status, 4) Social functioning, 5) Satisfaction with life in general, and 6) Satisfaction with health. A high score on each domain of the questionnaire represents a better quality of life. Internal consistency and construct validity have shown to be adequate.

\section{Visual Analogue scale (VAS)}

Self-report measure of pain intensity. Sliding scale where the ends of the line represent the extreme limits of pain intensity (no pain at all and worst pain imaginable). Average VAS score is taken of pain at this moment, worst and least pain in last week. Reliable method in children above 8 years old. Sound psychometric properties and clinical utility.

\section{Functional Disability Inventory - Parent report (FDI)}

Measure of parent perceived functional disability of their child. 15 items rated on a five-point scale (0-4). Total scores range $0-60$ with higher scores indicating greater parent-perceived difficulty in performing activities. Consists of subscales 1) Negative mood, 2) Interpersonal problems,
Baseline, 8 weeks, 16 weeks, 10 and 12 months

Baseline, 8 weeks, 16 weeks, 10 and 12 months

Baseline, 8 weeks, 16 weeks, 10 and 12 months

Baseline, 8 weeks, 16 weeks, 10 and 12 months

Baseline, 8 weeks, 16 weeks, 10 and 12 months 
Table 3 Primary and secondary outcome measures* Parent perceived
Fear of Pain

(48)

Parental Pain catastrophizing (50)

Adult response to children's symptoms (54)
Fear of Pain Questionnaire - Parent report (FOPQ-P)

Parent proxy report measure to assess parents perception

of their childs pain-related fear experience.

23 items rated on a five-point scale (0-4). Total scores range 0-92 with

higher scores indicating a higher parent perceived fear. Consists of subscales

1) fear of pain, 2) avoidance of activities, and 3) School avoidance.

Psychometrically sound measure with strong internal

consistency, good construct and criterion validity.

\section{Pain Catastrophizing Scale - Parent report (PCS-P)}

Measures parental catastrophizing about their child's pain

13 items rated on a five-point scale (0-4). Total scores range $0-52$ with

higher scores indicating more catastrophic thinking by the parents.

Psychometric properties are good to very good.

\section{Adult response to children's symptoms scale (ARCS)}

Parent self-report measure of a range of parental

responses to pain of their child.

29 items rated on a five-point scale (Never-always). Consists

of subscales 1) Protective responses, 2) minimizing responses

(criticizing or downplaying the pain) and 3) monitoring/encouraging

responses (encouraging activity while monitoring symptoms)

Valid instrument for assessing parents' responses to

children's pain for diverse chronic pain symptoms.

\begin{tabular}{l}
\hline $\begin{array}{l}\text { Other study } \\
\text { parameters }\end{array}$ \\
\hline $\begin{array}{l}\text { Costs } \\
\text { (economic evaluation) }\end{array}$ \\
Generic health \\
related quality of life \\
(economic evaluation) \\
(55)
\end{tabular}

Satisfaction/patient centeredness (Process evaluation) (57)

Satisfaction/family centeredness (Process evaluation) $(58,59)$

Treatment

expectations

of children

(Process evaluation)

(60)

Treatment

expectations parents

(Process evaluation)

(60)

Joint Hypermobility

Syndrome (HMS)

$(29,32,43)$
Baseline, 8 weeks,

16 weeks, 10

and 12 months

Baseline, 8 weeks,

16 weeks, 10

and 12 months

Baseline, 8 weeks,

16 weeks, 10

and 12 months

\section{Used in economic evaluation and process evaluation}

\section{Cost diary}

Health care utilization, school absence and productivity losses are recorded.

\section{EQ-5D-Y}

Generic self-report measure of health related quality of life.

5 Items, scored on three levels: no problems, moderate problems, severe

problems. Contains domains 1) mobility, 2) self-care, 3) usual activities,

4) pain/discomfort, and 5) anxiety/depression. 1 visual analogue scale

to rate their own health between 0 and 100 (best health state).

\section{Giving Youth A Voice Questionnaire (GYV-20)}

The instrument has 4 themes, 1) supportive and respectful relationships, 2) Information sharing and communication, 3) Supporting independence and 4) Teen-centred service. Each item is formulated as a question, starting with 'How much do the people who work with you...' and then a description of a specific action or behaviour of the health care professional is given. Response options range from 1-7, with a 'not applicable' category added. Scale scores can be calculated as the mean of the ratings for the items in the scale.

\section{Measure of Processes Of Care - Parent form - Short form (MPOC-P-20)}

20 items, scored on a scale 1-7. Consists of 5 scales, 1) Enabling and Partnership, 2) Providing General Information, 3) Providing Specific Information about the Child, 4) Coordinated and Comprehensive Care for the Child and Family and 5) Respectful and Supportive Care. Responses to each item are converted to a mean for each scale. No total score can be calculated.

\section{Time points of \\ measurement \\ After treatment \\ each month \\ until month 12 . \\ Baseline, 8 weeks, 16 weeks, 10 and 12 months}

16 weeks

16 weeks

Baseline

Credibility/Expectancy Questionnaire (CEQ-Adolescent)

One 11 items (5 on credibility, 6 on expectancy) can be answered

on a 9-point scale from 'totally not' to 'totally'. Total scores are a sum

score of the individual items and ranges from 11 to 99.

\section{Credibility/Expectancy Questionnaire (CEQ-Parent)}

Baseline

On the 11 items (5 on credibility, 6 on expectancy) can be answered

on a 9-point scale from 'totally not' to 'totally'. Total scores are a sum

score of the individual items and ranges from 11 to 99.

\section{Birghton Criteria}

To identify adolescents with HMS the Brighton criteria, including a Beighton

Baseline

*Not for all measures detailed information about reliability and validity was available. 


\section{Process Evaluation}

To evaluate treatment fidelity, a process evaluation will be performed. The Method of Assessing Treatment Delivery (MATD) (57) will be applied, including audio or videotaping treatment sessions to assess protocol adherence. There will be a focus on protocol adherence of the therapists in the MRP condition and a contamination check will be performed to evaluate whether treatments provided in both arms of the trial were indeed different from each other. For treatment fidelity to be adequate, at least $70 \%$ of essential treatment elements must have occurred in order to satisfy the requirements for protocol adherence. Furthermore, maximally $10 \%$ of prohibited treatment elements may have occurred to satisfy the requirements for contamination. Furthermore, satisfaction with the received treatment and the degree of adolescent/family centeredness of the offered interventions will be measured with the Giving Youth a Voice Questionnaire (GYV-20) (58) and Measure of Processes of Care Parent Version short form (MPOC-P-20) (59, 60). Finally, a comparison will be made between treatment expectation (measured with the Credibility/Expectancy Questionnaire, CEQ-m) (61).

\section{Data-analysis}

Statistical analysis

All analysis will be performed on the basis of the "intention-to-treat principle". Descriptive statistics for demographic and clinical characteristic for both groups (MRP and CAU) and the total group will be used. An inventory of missing data will be made and if necessary an imputation strategy will be chosen.

\section{Effect evaluation}

Differences after 8 and 16 weeks of treatment will be calculated (short term outcomes), as well as long term outcomes at 10 and 12 months. A linear mixed models approach for calculating differences between baseline and the final follow-up at 12 months will be used. This method used both fixed and random effects in the same analysis. It handles naturally unbalanced data as e.g. uneven spacing of repeated measures and allows analysing the relationship of predictor covariates with the dependent variable (FDI-score). It also accounts successfully for the observed pattern of dependences in the measurements. Appropriate covariates (for the primary outcome potential important imbalances in baseline variables) will be identified in a univariate regression analysis. Before starting the analysis the baseline score of the dependent variable FDI-score and all identified covariates will be centred by subtracting the group mean.

In a first step a fixed effects mode will be run and in a second step random effects will be added. Insignificant covariates will then be stepwise removed from the model. Model fit will be assessed with the help of the Bayesian Information Criterion (BIC) and the -2Log likelihood (62). 


\section{Economic evaluation}

In the economic evaluation costs and effects of the intervention condition (MRP) and the control condition (CAU) will be calculated and compared, using a societal perspective. Total costs will be estimated using a bottom-up approach, where information on each element of service use is multiplied by an appropriate standardized unit cost and summed to provide and overall total cost. For the cost valuation, standardized cost prizes will be used from the Dutch manual for cost analysis in health care research (63). Productivity losses will be calculated based on the Human Capital Approach.

At baseline resource use prior to the study will be measured, to allow this use as a covariate in the analysis, which enables us to correct for possible cost differences at baseline (64). Cost per patient year (= participant year) will be calculated.

Intended, both a cost-effectiveness analysis (CEA) and a cost-utility analysis (CUA) will be performed. For the CEA the cost-effectiveness ratio will be stated in terms of cost per 5 points of improvement on the Functional Disability Inventory (FDI). For the CUA the cost-utility ratio will be stated in terms of cost per Quality Adjusted Life Year (QALY) gained, as measured by the EQ5D-Y (65). Bootstrap re-sampling techniques will be used to test for differences and uncertainty in cost and effects between MRP and CAU.

\section{Process evaluation}

To evaluate treatment fidelity, audio or video recordings will be analysed of treatment sessions of both interventions conditions (42). From these recordings, a random sample will be taken for analysis. The recordings are rated for protocol adherence, contamination, and differentiation (42). Ratings will be performed by 2 raters, independent of this trial and blind to the study hypotheses. To enable rating, a Treatment Fidelity Checklist is developed where raters can indicate whether a treatment-element took place or not (57). The percentage of deviation from the allowed treatment-elements is compared between the intervention and control condition by logistic regression analysis.

The data from the Credibility/expectancy questionnaire and the measures of patient/family centeredness (GYV-20 and MPOC-20) of the provided treatment will be used to evaluate differences in credibility and expectancy at the start of the treatment, and treatment satisfaction at the end of treatment.

\section{DISCUSSION}

Research on treatment options for adolescents with CMP is still in its infancy, but multidisciplinary treatment for this patient group seems to be a promising treatment approach. A limited number of studies have been performed to study the effectiveness of multidisciplinary treatments on improving functional ability (66-70). Although several pre- and post-treatment comparisons have been 
performed, to our knowledge only few studies have compared different treatment options with each other. Another important element in adolescent treatment is the participation of parents, again, with limited evidence available on the effectiveness of parental interventions $(17,22,38)$.

There is an urgent need for more research. Therefore, a pragmatic multicentre randomized controlled trial (RCT) was established to compare a new multimodal rehabilitation program with care as usual. For this study a more pragmatic approach is chosen. Schwarts and Lellouch (71) use the term 'pragmatic'to describe studies designed to choose between options of care, as opposed to 'explanatory'studies that test causal research hypotheses. Our aim is to compare the new treatment approach to existing practice to determine whether or not the new approach can be added to the treatment options existing presently. This approach led to some design choices that were made to maximize the applicability of the results to usual care settings.

To include a wide range of participants eligibility criteria are focused on the inclusion of all adolescent participants that would normally receive an indication for rehabilitation treatment. This should lead to the inclusion of a heterogeneous group of participants, very similar to the patients that are seen in current clinical practice.

Adolescents aged 12-21 years old are eligible to participate in the trial. Paediatric rehabilitation services are in principle provided up to the age of 18 years old. Most studies on treatments for adolescents include participants up to the age of 18 years old. The range of 12-21 years was chosen according to Kaplan's (72) definition of adolescent life stage, starting with early adolescence at 12 years old, ending of late adolescence at 21 years old. Furthermore, research has shown that up to the age of 21 years, brain functions are still developing (73). Therefore, interpretation of cognitive and behavioural processes can still be sensitive to change and can best be seen in to context of adolescence instead of adulthood. Additionally, in the Netherlands only $20 \%$ of the 15-20 year olds are part of the working population, so for the largest part of this age group, their daily social and physical activities are more in line with the school-attending adolescents than with the working adult population.

To our knowledge, 2B Active is one of the first studies performing an economic evaluation on outpatient treatment and follow up, focussing both on the cost of the intervention itself and on patient and family costs. No measurement instrument existed that measured both adolescent and parent costs of the rehabilitation trajectory and follow-up. Therefore, a cost diary was developed to measure both medical consumption and productivity losses (work and school absenteeism) after completion of the rehabilitation program.

To summarize, several design choices that have been made may influence the balance between internal validity and external validity of the study. By studying the new intervention in a pragmatic way and in the setting in which the MRP treatment is intended to be offered, the results will be more generalizable to daily practice of rehabilitation care. Including a heterogeneous group of patients and analysis according to ITT may lead to results that are not over-estimated since real practice daily difficulties are incorporated in the trial results. 


\section{DECLARATIONS}

List of abbreviations CMP: Chronic musculoskeletal pain; MRP: Multimodal Rehabilitation Program; CAU: Care as Usual; HMS: Hypermobility syndrome; RCT: Randomized Controlled Trial; GA: Graded Activity; GE: Graded Exposure; FDI: Functional Disability Inventory; FOPQ-C: Fear of Pain Questionnaire- Child version; PHODA-Youth: Photograph Series of Daily Activities for youth; PCSC: Pain Catastrophizing Scale - Child version; VAS: Visual Analogue Scale; CDI: Child Depression Inventory; QLA-CP: Quality of Life in Adolescents with Chronic Pain; FDI-P: Functional Disability Inventory-Parent report; FOPQ-P: Fear of Pain Questionnaire-Parent report; PCS-P: Pain Catastrophizing Scale-Parent version; ARCS: Adult responses to Children's Symptoms scale; MATD: Method of Assessing Treatment Delivery; GYV-20: Giving Youth a Voice Questionnaire; MPOC-P-20: Measure of Processes of Care Parent version short form; CEQ: Credibility/Expectancy Questionnaire; CEA: Cost-Effectiveness Analysis; CUA: Cost-Utility Analysis; QALY: Quality Adjusted Life Year

Ethics approval and consent to participate Ethical approval for this trial was granted by the Medical Ethics Committee Academic Hospital Maastricht/Maastricht University, the Netherlands, NL47323.068.13/METC13-3-062. Participants can only be included in the study after informed consent has been obtained. For participants under 18 years old, consent for their participation was obtained from their parents or legal guardian as well as their own consent.

Consent for publication Not applicable.

Availability of data and materials Not applicable.

Competing interests The authors declare that they have no competing interests.

Authors' contributions CD wrote the first draft of the manuscript, designed the study and co-ordinated the RCT. MEJBG conceived and designed the study, led the project, commented on drafts of the manuscript. CHGB designed the study with a specific focus on the methodology used and reviewed the manuscript. JAMCFV conceived and designed the study and commented on drafts of the manuscript. All authors read and approved the final manuscript.

\section{ACKNOWLEDGEMENTS}

We acknowledge Marion de Mooij for her assistance on this research project, Jeroen de Jong for his advice on Graded Exposure therapy and training the Graded Exposure therapists participating in this RCT, Emmelien Spek, Thijs van Meulenbroek and Debby van Menxel for their input in developing the treatment protocols and all collaborating treatment centres and participants participating in this project. 


\section{REFERENCES}

1. Perquin CW, Hazebroek-Kampschreur AAJM, Hunfeld JAM, Bohnen AM, van Suijlekom-Smit LWA, Passchier J, et al. Pain in children and adolescents: a common experience. Pain. 2000;87(1):51-8.

2. Eccleston C, Bruce E, Carter B. Chronic pain in children and adolescents. Paediatr Nurs. 2006;18(10):30-3.

3. King S, Chambers CT, Huguet A, MacNevin RC, McGrath PJ, Parker L, et al. The epidemiology of chronic pain in children and adolescents revisited: A systematic review. Pain. 2011;152(12):2729-38.

4. Perquin CW, Hazebroek-Kampschreur AA, Hunfeld JA, van Suijlekom-Smit LW, Passchier J, van der Wouden JC. Chronic pain among children and adolescents: physician consultation and medication use. Clin J Pain. 2000;16(3):229-35.

5. El-Metwally A, Salminen JJ, Auvinen A, Kautiainen H, Mikkelsson M. Prognosis of non-specific musculoskeletal pain in preadolescents: a prospective 4-year follow-up study till adolescence. Pain. 2004;110(3):550-9.

6. Kashikar-Zuck S. Treatment of children with unexplained chronic pain. Lancet. 2006;367(9508):380-2.

7. McGhee JL, Burks FN, Sheckels JL, Jarvis JN. Identifying children with chronic arthritis based on chief complaints: absence of predictive value for musculoskeletal pain as an indicator of rheumatic disease in children. Pediatrics. 2002;110(2 Pt 1):354-9.

8. Mikkelsson M, El-Metwally A, Kautiainen H, Auvinen A, Macfarlane GJ, Salminen JJ. Onset, prognosis and risk factors for widespread pain in schoolchildren: a prospective 4-year follow-up study. Pain. 2008;138(3):681-7.

9. Martin AL, McGrath PA, Brown SC, Katz J. Children with chronic pain: impact of sex and age on longterm outcomes. Pain. 2007;128(1-2):13-9.

10. Fearon P, Hotopf M. Relation between headache in childhood and physical and psychiatric symptoms in adulthood: national birth cohort study. BMJ. 2001;322(7295):1145.

11. Merlijn VP, Hunfeld JA, van der Wouden JC, Hazebroek-Kampschreur AA, Passchier J, Koes BW. Factors related to the quality of life in adolescents with chronic pain. Clin J Pain. 2006;22(3):306-15.

12. Konijnenberg AY, Uiterwaal CS, Kimpen JL, van der Hoeven J, Buitelaar JK, de Graeff-Meeder ER. Children with unexplained chronic pain: substantial impairment in everyday life. Arch Dis Child. 2005;90(7):680-6.

13. Huguet A, Miro J. The severity of chronic pediatric pain: an epidemiological study. J Pain. 2008;9(3):226-36.

14. Hunfeld JA, Passchier J, Perquin CW, Hazebroek-Kampschreur AA, van Suijlekom-Smit LW, van der Wouden JC. Quality of life in adolescents with chronic pain in the head or at other locations. Cephalalgia. 2001;21(3):201-6.

15. Palermo TM, Eccleston C. Parents of children and adolescents with chronic pain. Pain. 2009;146(12):15-7.

16. Eccleston C, Crombez G, Scotford A, Clinch J, Connell H. Adolescent chronic pain: patterns and predictors of emotional distress in adolescents with chronic pain and their parents. Pain. 2004;108(3):221-9.

17. Hechler T, Ruhe AK, Schmidt P, Hirsch J, Wager J, Dobe M, et al. Inpatient-based intensive interdisciplinary pain treatment for highly impaired children with severe chronic pain: randomized controlled trial of efficacy and economic effects. Pain. 2014;155(1):118-28. 
18. Sleed M, Eccleston C, Beecham J, Knapp M, Jordan A. The economic impact of chronic pain in adolescence: methodological considerations and a preliminary costs-of-illness study. Pain. 2005;119(13):183-90.

19. Groenewald CB, Essner BS, Wright D, Fesinmeyer MD, Palermo TM. The economic costs of chronic pain among a cohort of treatment-seeking adolescents in the United States. J Pain. 2014;15(9):925-33.

20. Lynch-Jordan AM, Kashikar-Zuck S, Szabova A, Goldschneider KR. The interplay of parent and adolescent catastrophizing and its impact on adolescents' pain, functioning, and pain behavior. Clin J Pain. 2013;29(8):681-8.

21. Logan DE, Simons LE, Carpino EA. Too sick for school? Parent influences on school functioning among children with chronic pain. Pain. 2012;153(2):437-43.

22. Palermo TM, Valrie CR, Karlson CW. Family and parent influences on pediatric chronic pain: a developmental perspective. Am Psychol. 2014;69(2):142-52.

23. Caes L, Vervoort T, Eccleston C, Vandenhende M, Goubert L. Parental catastrophizing about child's pain and its relationship with activity restriction: the mediating role of parental distress. Pain. 2011;152(1):212-22.

24. Guite JW, McCue RL, Sherker JL, Sherry DD, Rose JB. Relationships among pain, protective parental responses, and disability for adolescents with chronic musculoskeletal pain: the mediating role of pain catastrophizing. Clin J Pain. 2011;27(9):775-81.

25. Goubert L, Simons LE. Cognitive styles and processes in paediatric pain. In: McGrath PA, Stevens BJ, Walker SM, Zempsky WT, editors. Oxford Textbook of Paediatric Pain. Oxford: Oxford University Press; 2014. p. 95-101.

26. Goossens M, Nijhuis A, Vikstroem M, Verbunt J. F274 THE PSYCHOMETRIC QUALITIES OF THE PHODA-YOUTH FOR ADOLESCENTS WITH CHRONIC PAIN. Eur J Pain Suppl. 2011;5(S1):145-6.

27. Verbunt JA, Nijhuis A, Vikstrom M, Stevens A, Haga N, de Jong J, et al. The psychometric characteristics of an assessment instrument for perceived harmfulness in adolescents with musculoskeletal pain (PHODA-youth). Eur J Pain. 2015;19(5):695-705.

28. Kirk JA, Ansell BM, Bywaters EG. The hypermobility syndrome. Musculoskeletal complaints associated with generalized joint hypermobility. Ann Rheum Dis. 1967;26(5):419-25.

29. Grahame R, Bird HA, Child A. The revised (Brighton 1998) criteria for the diagnosis of benign joint hypermobility syndrome (BJHS). J Rheumatol. 2000;27(7):1777-9.

30. de Inocencio J. Musculoskeletal pain in primary pediatric care: analysis of 1000 consecutive general pediatric clinic visits. Pediatrics. 1998;102(6):E63.

31. Gedalia A, Press J, Klein M, Buskila D. Joint hypermobility and fibromyalgia in schoolchildren. Ann Rheum Dis. 1993;52(7):494-6.

32. Grahame R. Joint hypermobility syndrome pain. Current pain and headache reports. 2009;13(6):427-33.

33. Baeza-Velasco C, Gely-Nargeot MC, Bulbena Vilarrasa A, Bravo JF. Joint hypermobility syndrome: problems that require psychological intervention. Rheumatol Int. 2011;31(9):1131-6.

34. Vlaeyen JW, Linton SJ. Fear-avoidance and its consequences in chronic musculoskeletal pain: a state of the art. Pain. 2000;85(3):317-32.

35. Simons LE, Kaczynski KJ. The Fear Avoidance model of chronic pain: examination for pediatric application. The Journal of Pain 2012;13(9):827-35. 
36. Simons LE, Sieberg CB, Pielech M, Conroy C, Logan DE. What does it take? Comparing intensive rehabilitation to outpatient treatment for children with significant pain-related disability. J Pediatr Psychol. 2013;38(2):213-23.

37. Simons LE, Kaczynski KJ, Conroy C, Logan DE. Fear of pain in the context of intensive pain rehabilitation among children and adolescents with neuropathic pain: associations with treatment response. The Journal of Pain. 2012;13(12):1151-61.

38. Eccleston C, Fisher E, Law E, Bartlett J, Palermo TM. Psychological interventions for parents of children and adolescents with chronic illness. The Cochrane database of systematic reviews. 2015;4:CD009660.

39. Leeuw M, Goossens ME, van Breukelen GJ, de Jong JR, Heuts PH, Smeets RJ, et al. Exposure in vivo versus operant graded activity in chronic low back pain patients: results of a randomized controlled trial. Pain. 2008;138(1):192-207.

40. de Jong JR, Vlaeyen JW, Onghena P, Cuypers C, den Hollander M, Ruijgrok J. Reduction of pain-related fear in complex regional pain syndrome type I: the application of graded exposure in vivo. Pain. 2005;116(3):264-75.

41. Altman DG, Bland JM. Treatment allocation by minimisation. BMJ. 2005;330(7495):843.

42. Leeuw M. SAFE but SORRY, Theory, assesment, and treatment of pain-related fear in chornic low back pain patients. Maastricht: Universitaire Pers Maastricht; 2008.

43. Tinkle BT, Bird HA, Grahame R, Lavallee M, Levy HP, Sillence D. The lack of clinical distinction between the hypermobility type of Ehlers-Danlos syndrome and the joint hypermobility syndrome (a.k.a. hypermobility syndrome). Am J Med Genet A. 2009;149A(11):2368-70.

44. Ruijgrok JM, de Jong JR, Cuijpers CJT, den Hollander ML, Loo CAMJ. Nieuwe behandelmethode bij chronische pijn:'graded exposure in vivo'Tijdschrift voor Geneeskundig Adviseurs in particuliere verzekeringszaken. 2007;11(2):37-9.

45. McGrath PJ, Walco GA, Turk DC, Dworkin RH, Brown MT, Davidson K, et al. Core outcome domains and measures for pediatric acute and chronic/recurrent pain clinical trials: PedIMMPACT recommendations. J Pain. 2008;9(9):771-83.

46. Claar RL, Walker LS. Functional assessment of pediatric pain patients: psychometric properties of the functional disability inventory. Pain. 2006;121(1-2):77-84.

47. Walker LS, Greene JW. The functional disability inventory: measuring a neglected dimension of child health status. J Pediatr Psychol. 1991;16(1):39-58.

48. Simons LE, Sieberg CB, Carpino E, Logan D, Berde C. The Fear of Pain Questionnaire (FOPQ): Assessment of Pain-Related Fear Among Children and Adolescents With Chronic Pain. The Journal of Pain. 2011;12(6):677-86.

49. Crombez G, Bijttebier P, Eccleston C, Mascagni T, Mertens G, Goubert $L$, et al. The child version of the pain catastrophizing scale (PCS-C): a preliminary validation. Pain. 2003;104(3):639-46.

50. Goubert L, Eccleston C, Vervoort T, Jordan A, Crombez G. Parental catastrophizing about their child's pain. The parent version of the Pain Catastrophizing Scale (PCS-P): A preliminary validation. Pain. 2006;123(3):254-63.

51. Stinson JN, Kavanagh T, Yamada J, Gill N, Stevens B. Systematic review of the psychometric properties, interpretability and feasibility of self-report pain intensity measures for use in clinical trials in children and adolescents. Pain. 2006;125(1-2):143-57. 
52. Roelofs J, Braet C, Rood L, Timbremont B, van Vlierberghe L, Goossens L, et al. Norms and screening utility of the Dutch version of the Children's Depression Inventory in clinical and nonclinical youths. Psychol Assess. 2010;22(4):866-77.

53. Merlijn VP, Hunfeld JA, van der Wouden JC, Hazebroek-Kampschreur AA, Passchier J. Shortening a quality of life questionnaire for adolescents with chronic pain and its psychometric qualities. Psychol Rep. 2002;90(3 Pt 1):753-9.

54. Claar RL, Guite JW, Kaczynski KJ, Logan DE. Factor structure of the Adult Responses to Children's Symptoms: validation in children and adolescents with diverse chronic pain conditions. Clin J Pain. 2010;26(5):410-7.

55. Goossens ME, Rutten-van Molken MP, Vlaeyen JW, van der Linden SM. The cost diary: a method to measure direct and indirect costs in cost-effectiveness research. J Clin Epidemiol. 2000;53(7):688-95.

56. Wille N, Badia X, Bonsel G, Burstrom K, Cavrini G, Devlin N, et al. Development of the EQ-5D-Y: a child-friendly version of the EQ-5D. Qual Life Res. 2010;19(6):875-86.

57. Leeuw M, Goossens ME, de Vet HC, Vlaeyen JW. The fidelity of treatment delivery can be assessed in treatment outcome studies: a successful illustration from behavioral medicine. J Clin Epidemiol. 2009;62(1):81-90.

58. Siebes RC, Wijnroks L, Ketelaar M, van Schie PE, Vermeer A, Gorter JW. Validation of the Dutch Giving Youth a Voice Questionnaire (GYV-20): a measure of the client-centredness of rehabilitation services from an adolescent perspective. Disabil Rehabil. 2007;29(5):373-80.

59. King S, King G, Rosenbaum P. Evaluating health service delivery to children with chronic conditions and their families: Development of a refined measure of processes of care (MPOC-20). Child Health Care. 2004;33(1):35-57.

60. Siebes RC, Maassen GH, Wijnroks L, Ketelaar M, van Schie PE, Gorter JW, et al. Quality of paediatric rehabilitation from the parent perspective: validation of the short Measure of Processes of Care (MPOC-20) in the Netherlands. Clin Rehabil. 2007;21(1):62-72.

61. Devilly GJ, Borkovec TD. Psychometric properties of the credibility/expectancy questionnaire. J Behav Ther Exp Psychiatry. 2000;31(2):73-86.

62. Landau S, Everitt BS. A handbook of statistical analysis using SPSS. Florida: Chapmand \& Hall/CRC; 2004.

63. Hakkaart-van Roijen L, Tan SS, Bouwmans CA. Handleiding voor kostenonderzoek. Methoden en standaardkostprijzen voor economische evaluaties in de gezondheidszorg. College voor zorgverzekeringen, 2010.

64. van Asselt AD, van Mastrigt GA, Dirksen CD, Arntz A, Severens JL, Kessels AG. How to deal with cost differences at baseline. Pharmacoeconomics. 2009;27(6):519-28.

65. Horsman J, Furlong W, Feeny D, Torrance G. The Health Utilities Index (HUI): concepts, measurement properties and applications. Health and quality of life outcomes. 2003;1:54.

66. Sherry DD, Wallace CA, Kelley C, Kidder M, Sapp L. Short- and long-term outcomes of children with complex regional pain syndrome type I treated with exercise therapy. Clin J Pain. 1999;15(3):218-23.

67. Eccleston C, Malleson PN, Clinch J, Connell H, Sourbut C. Chronic pain in adolescents: evaluation of a programme of interdisciplinary cognitive behaviour therapy. Arch Dis Child. 2003;88(10):881-5.

68. de Blecourt AC, Schiphorst Preuper HR, Van Der Schans CP, Groothoff JW, Reneman MF. Preliminary evaluation of a multidisciplinary pain management program for children and adolescents with chronic musculoskeletal pain. Disabil Rehabil. 2008;30(1):13-20. 
69. Hechler T, Dobe M, Kosfelder J, Damschen U, Hubner B, Blankenburg M, et al. Effectiveness of a 3-week multimodal inpatient pain treatment for adolescents suffering from chronic pain: statistical and clinical significance. Clin J Pain. 2009;25(2):156-66.

70. Maynard CS, Amari A, Wieczorek B, Christensen JR, Slifer KJ. Interdisciplinary behavioral rehabilitation of pediatric pain-associated disability: retrospective review of an inpatient treatment protocol. J Pediatr Psychol. 2010;35(2):128-37.

71. Schwartz D, Lellouch J. Explanatory and pragmatic attitudes in therapeutical trials. J Chronic Dis. 1967;20(8):637-48.

72. Kaplan PS. Adolescence. Boston: Houghton Miffin Company; 2004.

73. Giedd JN, Blumenthal J, Jeffries NO, Castellanos FX, Liu H, Zijdenbos A, et al. Brain development during childhood and adolescence: a longitudinal MRI study. Nat Neurosci. 1999;2(10):861-3. 


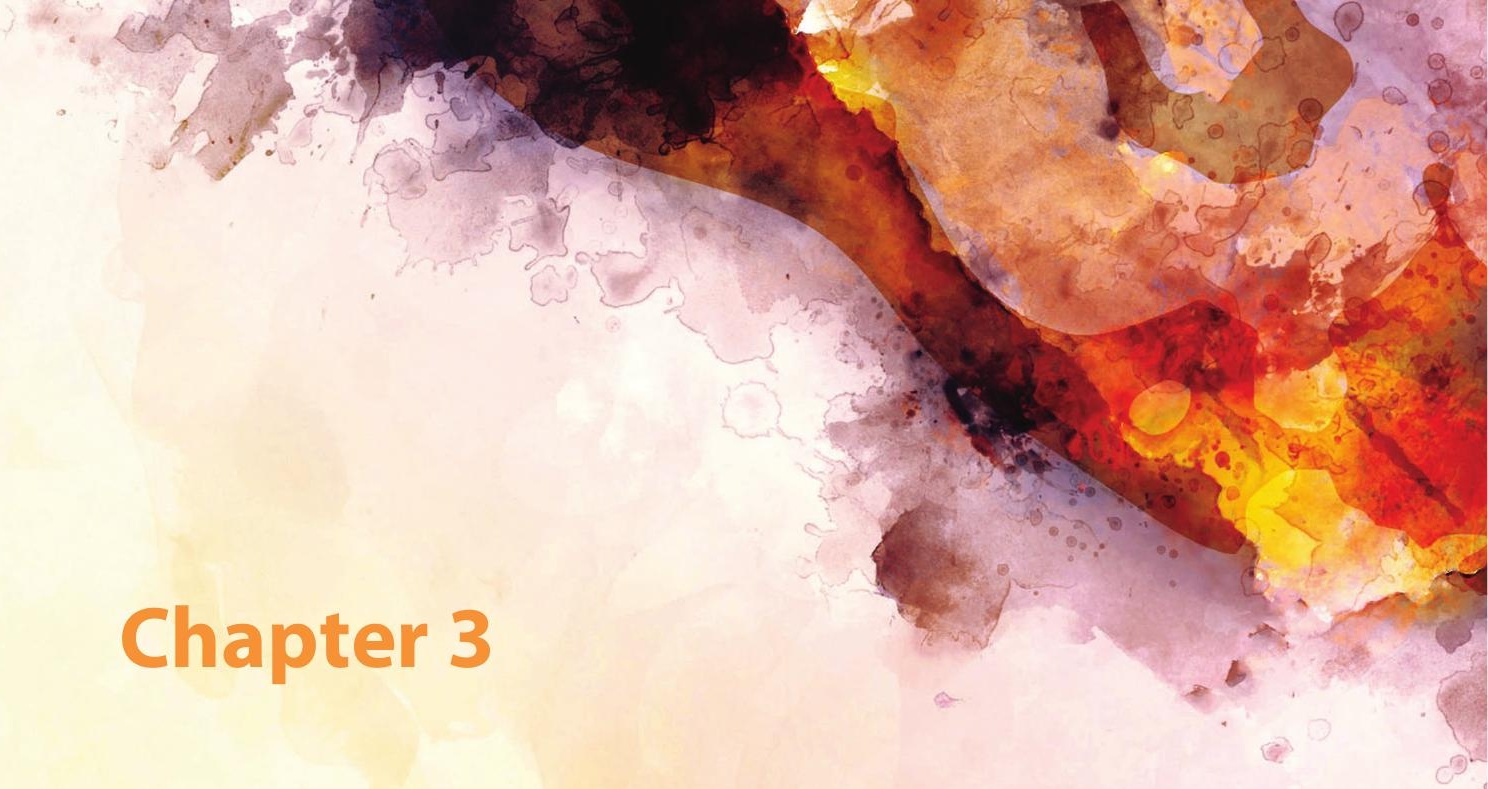

Functional disability in adolescents with chronic pain: comparing an interdisciplinary exposure program to usual care A randomized clinical trial

Carolien Dekker Mariëlle Goossens

Bjorn Winkens Sylvia Remerie Caroline Bastiaenen Jeanine Verbunt 


\section{ABSTRACT}

Importance Chronic musculoskeletal pain (CMP) in adolescents can negatively affect physical, psychological and social functioning. An interdisciplinary graded exposure program (EP) was developed to reduce adolescent functional disability.

Objective To evaluate the effectiveness of EP in reducing functional disability in adolescents with CMP, compared with care as usual (CAU).

Design A pragmatic multicenter randomized clinical trial, recruiting between August 2014 and September 2016 with a 12-month follow-up. Data analysis was by intention-to-treat linear mixed model analysis.

Setting Specialized rehabilitation care in four rehabilitation centers in the Netherlands.

Participants Adolescents aged 12-21 years with CMP, reporting pain-related fear and referred to outpatient rehabilitation treatment, were eligible to participate. Exclusion criteria were: a medical disease explaining pain; an underlying psychiatric disease hampering rehabilitation; and pregnancy. Of 77 invited participants, 60 were randomized to EP or CAU (1:1) after giving informed consent. Interventions EP is an interdisciplinary individual outpatient rehabilitation program, with an adolescent and a parent module. The program uses graded exposure in vivo to improve functional ability by reducing pain-related fear. CAU is interdisciplinary outpatient rehabilitation care, based on the principles of graded activity. Treatment duration varies between 7 and 16 weeks in both interventions.

Main outcomes and measures Primary outcome was functional disability measured with the Functional Disability Inventory. Protocol adherence by treatment teams was evaluated with the Method of Assessing Treatment Delivery.

Results 53 adolescents (93\% female) were analyzed (25 EP, 28 CAU). Mean age was 16.0 years $(S D=1.87)$. Adolescents in EP showed a clinically relevant and statistically significant decrease in functional disability (estimated mean difference at least -8.81, p-values $\leq 0.01$ ) compared with CAU at all time points. Protocol adherence by treatment teams in EP was high and contamination by the alternative intervention was low.

Conclusions and relevance In adolescents with CMP, EP leads to a significantly larger and clinically relevant decrease in functional disability than usual care. Therefore, implementation of EP in rehabilitation care is recommended.

Trial registration Clinicaltrials.gov ID: NCT02181725 


\section{KEYPOINTS}

Question The aim of this study was to investigate the effectiveness of an interdisciplinary graded exposure program in reducing functional disability in adolescents with chronic musculoskeletal pain, compared with care as usual.

Findings $A$ randomized controlled clinical trial with 60 adolescents with chronic musculoskeletal pain demonstrated a clinically relevant and statistically significant difference of at least -8.8 points in functional disability, in favor of the exposure program, from end of treatment to follow-up at up to 12 months.

Meaning For adolescents with chronic musculoskeletal pain reporting pain-related fear, an interdisciplinary graded exposure program is recommended to reduce functional disability. 


\section{INTRODUCTION}

Chronic pain in children and adolescents is a major health concern (1, 2). Chronic musculoskeletal pain (CMP) is one of the most reported pain complaints next to chronic headache and abdominal pain $(1,2)$. Internationally, prevalence rates for CMP vary between 4 and $40 \%$ and appear to be increasing $(1,2)$. Pain during adolescence increases the risk of pain in adulthood $(3,4)$.

Evidence about treatment effects of interdisciplinary treatments for adolescent chronic pain is relatively scarce. Psychologically-based treatments, including cognitive behavioral therapy, for adolescent chronic pain appear to be effective in reducing pain, and the quality of studies has improved over the years $(5,6)$. However, most studies focus on adolescents with headache and use pain reduction as the primary outcome of interest. Evidence of treatment effectiveness on disability and emotional distress in adolescents with CMP is rare (5-7).

Pain-related fear can contribute to the development and maintenance of chronic pain and, in adults, there is evidence that cognitive behavioral graded exposure in vivo decreases functional disability by reducing pain-related fear (8-10). The fear-avoidance model of chronic pain is the theoretical model underlying exposure therapy (11). According to this model, in the event of pain, both fear of pain/movement and catastrophic thinking about pain can lead to the development and maintenance of chronic pain problems (11). By exposing patients to movements and activities previously avoided due to pain-related fear, patients find that normal functioning is possible despite pain (8-10).

Recently, more evidence was found on the negative consequences of pain-related fear in children and adolescents with chronic pain (12-14). Furthermore, the fear-avoidance model was expanded into an interpersonal fear avoidance model, accounting for the interaction between adolescents and parents, the social context in which adolescent pain problem arises (15). These progresses led to the development of an interdisciplinary graded exposure program (EP), specifically for adolescents with CMP and their parents. Furthermore, physical training $(16,17)$ was added to the exposure program for adolescents who were diagnosed with joint hypermobility syndrome, to prevent hypermobility problems hindering the graded exposure in vivo.

The primary objective of this study is evaluating the effectiveness of the exposure program in reducing functional disability, compared with care as usual, in adolescents of 12-21 years with CMP reporting pain-related fear. Secondary objectives are evaluating the effectiveness of the EP in reducing fear of pain, perceived harmfulness, pain catastrophizing, depressive symptoms, pain intensity, and improving health related quality of life. Further, delivery of treatment is evaluated by checking protocol adherence of the treatment teams in EP and seeing whether contamination by the alternative intervention has occurred. 


\section{METHODS}

\section{Study design}

The design was a multicenter pragmatic randomized controlled clinical trial (RCT) to evaluate whether EP is superior to CAU in reducing functional disability, the primary outcome measure. Ethical approval was granted for this trial (NL47323.068.13). A study protocol is published elsewhere (18). A pragmatic approach was chosen to allow potential future users of the interventions to choose between options of care (19), once the results are known.

\section{Participants, recruitment, eligibility criteria, and centers}

Adolescents were recruited by consultants in rehabilitation medicine in four Dutch rehabilitation centers between August 2014 and September 2016. Recruitment was after a pre-treatment screening and after eligibility criteria were checked. Adolescents referred to outpatient rehabilitation treatment for CMP, reporting pain-related fear according to the professional opinion of the interdisciplinary treatment team, aged 12-21 years, and with adequate Dutch literacy, were eligible for inclusion. Exclusion followed in case of any suspicion of a medical (orthopedic, rheumatic or neurological) disease that could fully explain their current level of severity of pain complaints, or any suspicion of an underlying psychiatric disease that would hamper rehabilitation treatment, or pregnancy.

Two of the four rehabilitation centers were rehabilitation departments of hospitals offering specialized outpatient rehabilitation care. All centers offered EP and CAU. A coordinator was appointed in each center to support the treatment teams with the study procedures during the trial.

\section{Interventions}

The exposure program (EP) consisted of active treatment sessions for both the adolescents and their parents. This program aims to restore the adolescents' age-appropriate functional abilities by systematically reducing pain-related fear and catastrophic thinking through gradually exposing adolescents to fear-provoking daily activities and movements. For adolescents, the treatment entailed an intake session with a consultant in rehabilitation medicine, screening, and 14 program sessions of 60 minutes each, during a seven-week period. The program sessions comprised an interdisciplinary intake session, an education session, and twelve graded exposure in vivo sessions. For parents, three parent meetings were offered in parallel with their adolescents' program, in a group or individually. For adolescents diagnosed with joint hypermobility syndrome, the program incorporated 16 physical training sessions of 120 minutes each. Training was offered prior to the graded exposure in vivo sessions, expanding program duration to 15 weeks. A detailed description of EP is provided elsewhere $(18,20)$.

The control condition was care as usual (CAU), predominantly interdisciplinary cognitive behavioral graded activity treatment. In graded activity treatment, the aim is to restore the adolescents' age-appropriate functional abilities by encouraging desired behaviors, and a time-con- 
tingent, stepwise increase in activity levels. For the control intervention, centers followed their own CAU protocol, which is based on a consensus document of the Dutch working group for youth with chronic pain and fatigue. Due to each center's practical and logistic differences, CAU treatment duration varied between 9-16 weeks.

Both interventions were specialized rehabilitation care offered by interdisciplinary treatment teams consisting of a consultant in rehabilitation medicine, a psychologist, and a physiotherapist or occupational therapist. In CAU, a social worker might be involved as well. In both interventions, adolescents were asked to refrain from other (co-)interventions, and medication use was reduced or terminated if possible. In both interventions, an individual treatment plan was proposed to adolescents and parents, the teams evaluated progress regularly, the consultant in rehabilitation medicine evaluating progress with the adolescents on their treatment.

\section{Data collection and outcome measures for treatment effectiveness}

Measurements were at baseline and at 2, 4, 10 and 12 months after start of EP, by digital questionnaires, accessible through a personalized link sent by email. Description of the measures is provided elsewhere (18).

The primary outcome was functional disability, measured with the Functional Disability Inventory (FDI, 15 items, scored on a 0-4 point Likert scale: total score range 0-60, higher scores indicating more severe disability) $(21,22)$. Secondary outcomes were fear of pain (Fear of Pain Questionnaire) $(23,24)$, perceived harmfulness (Photograph Series of Daily Activities for adolescents) (25), pain catastrophizing (Pain Catastrophizing Scale) (26), depressive symptoms (Children's Depression Inventory) (27), pain intensity (Visual Analogue Scale) (28), and pain-specific quality of life (Quality of Life Questionnaire for Adolescents with Chronic Pain) (29).

\section{Data collection and outcome measures to verify protocol adherence of the EP and contamination}

An adapted Method of Assessing Treatment Delivery (MATD) was used $(20,30)$ to measure protocol adherence in EP and verify that neither intervention was contaminated with elements of the other intervention. Protocol adherence was measured as the degree to which essential treatment elements of EP were offered by the treatment teams $(30,31)$. Treatment teams recorded their own program sessions. A random sample of 36 audio- and video-recorded sessions (14\% of 262 recorded sessions) was drawn for analysis. Outcomes were reported as percentages for protocol adherence and contamination.

\section{Randomization, allocation concealment and blinding}

Minimization was used. Minimization factors chosen were age, sex and treatment center. In each center, the first adolescent had a 50\% probability of being allocated to EP or CAU. In case of an unbalance in minimization factors, the probability of allocation to a particular group was adjusted 
to $90 \%$ for each following adolescent, to better ensure balance. The procedure was executed by a validated electronic randomization system (ALEA, offered by the Clinical Trial Center Maastricht). After written informed consent, the site coordinator was able to insert participant data: the system then randomized the adolescent and arranged a blinded treatment allocation. Thus, randomization and treatment allocation were completely independent of the study.

After allocation, blinding of the treatment group could not be maintained for treatment teams and site coordinators. Adolescents and parents were not notified of the details of the treatment comparison. The research team remained blinded during data collection and analysis.

\section{Sample size}

Sample size was calculated for the primary outcome measure FDI. A mean of 23 points (SD $=9.2$ ) (own unpublished clinical data) and expected mean difference of 5 points (approximately 25\% difference) between groups on the total average FDI score at the end of treatment were used. Given $a=0.05$, two sided testing, a power of $80 \%$, and anticipating $15 \%$ loss to follow-up, a sample size of 62 participants per trial arm, 124 participants in total was calculated.

\section{Statistical analysis}

Descriptive statistics were used to explore the data, check for outliers and summarize baseline characteristics (number, \% or observed mean, SD) for adolescents. Analyses were performed in IBM SPSS Statistics for Windows, version 25 (Armonk, NY: IBM Corp.).

\section{Analysis of treatment effectiveness}

To evaluate effectiveness, intention-to-treat linear mixed model analysis was used. This analysis accounts for correlation between repeated measures, uses all available data, assumes missing values to be random (missing at random, MAR), and corrects for baseline differences. Since it uses a likelihood approach, no imputation strategy was used. The primary and secondary outcome measures were used as dependent variables, while time (categorical: 0, 2, 4, 10, and 12 months), group (intervention vs control), interaction between time and group, and minimization variables (age, sex, and center) were included as fixed factors. If necessary, variables related to missing outcome values were included in the fixed part of the model to ensure MAR. As for the random part of the model, several options were considered, including an unstructured (UN) covariance structure for repeated measures, or a random intercept and/or random slope model (unstructured or variance components). The model with the smallest Bayesian Information Criterion (BIC) was chosen to be the best fitting model. Effect sizes are reported as estimated mean differences with 95\% confidence interval between intervention and control. Two-sided $p$-values $\leq 0.05$ were considered statistically significant. 
Analysis of treatment delivery

The recordings of treatment sessions were scored by two independent raters. In case of sufficient inter-rater reliability (Cohen's Kappa $\geq 0.61$ ) (32), mean scores of both raters were used for subsequent analysis. Following the criteria of Leeuw and colleagues (30), for the EP, the proportion of essential treatment elements present in three different program phases (preparation, education, treatment) should exceed $70 \%$ for sufficient protocol adherence. Contamination was considered absent when less than 10\% of prohibited treatment elements were scored in both treatments. Furthermore, more than $90 \%$ of the recorded sessions should be classified correctly as belonging to either EP or CAU.

\section{RESULTS}

\section{Description of the study population}

Seventy-seven eligible adolescents were invited to participate. Seventeen participants declined participation for different reasons. Sixty adolescents were randomized and, because of seven completely missing cases, 53 were analyzed (Figure 1). In Table 1 adolescent characteristics and baseline scores on the outcome measures are reported. Mean age was 16.0 years $(S D=1.87$, range 12-20 years), 49 (92\%) adolescents were female. At baseline, there were no meaningful differences between the groups. The recruitment period was extended by 7 months from the planned 1.5 years.

\section{Effects of the multimodal rehabilitation program}

Table 2 shows treatment effects of EP compared with CAU. No variables were significantly related to missing values in the outcome measures at any time point. For all dependent variables, a random intercept model was the best fitting model.

For the primary outcome FDI, estimated mean differences of at least 8.8 points ( $p$-values $\leq$ 0.011 ) between EP and CAU, in favor of EP, were observed for all time points, corrected for baseline (Table 2, Figure 2).

On the secondary outcomes, significant differences in favor of EP were found for perceived harmfulness at all time points ( $p$-values $\leq 0.002$ ), for pain catastrophizing (PCS) at 2 months follow-up ( $p$-value $=0.039$ ), for depressive symptoms at 10 months follow-up ( $p$-value $=0.008$ ), for pain intensity at 4 and 10 months follow-up ( $p$-values $\leq 0.028$ ), for quality of life for the domain Psychological Functioning at 2 and 10 months follow-up ( $p$-value $\leq 0.044$ ), and for the domain Functional Status at 2 and 4 months follow-up ( $p$-values $\leq 0.016$ ). 
Table 1 Characteristics of study participants at baseline ( $n=53, n=7$ missing)

\begin{tabular}{|c|c|c|c|}
\hline & $\begin{array}{l}\text { Exposure program } \\
\left(n=25^{* *}\right)\end{array}$ & $\begin{array}{l}\text { Care as Usual } \\
\left(n=28^{* *}\right)\end{array}$ & $\begin{array}{l}\text { Total } \\
(n=53)\end{array}$ \\
\hline Age (years) - mean (SD) & $15.9(1.99)$ & $16.2(1.79)$ & $16.0(1.87)$ \\
\hline Sex (female) - $n(\%)$ & $24(96 \%)$ & $25(89 \%)$ & $49(92 \%)$ \\
\hline Relative with pain complaints $-n\left(\%^{*}\right)$ & $13(62 \%)$ (4 missing) & 15 (60\%) (3 missing) & $28(61 \%)$ \\
\hline Other health issues $-\mathrm{n}\left(\%^{*}\right)$ & $8(38 \%)$ (4 missing) & 11 (44\%) (3 missing) & $19(41 \%)$ \\
\hline Onset of current pain complaints $-n\left(\%^{*}\right)$ & (4 missing) & (4 missing) & (8 missing) \\
\hline$<1$ year & $5(24 \%)$ & $12(50 \%)$ & $17(38 \%)$ \\
\hline $1-5$ years ago & $14(67 \%)$ & $11(46 \%)$ & $25(56 \%)$ \\
\hline$>5$ years ago & $2(10 \%)$ & $1(4 \%)$ & $3(7 \%)$ \\
\hline Problems with sleep - $n(\%)$ & 14 (67\%) (4 missing) & 17 (68\%) (3 missing) & $31(67 \%)(7$ missing $)$ \\
\hline Education-n $(\% *)$ & (3 missing) & (3 missing) & (6 missing) \\
\hline Low & $11(55 \%)$ & $16(64 \%)$ & $27(58 \%)$ \\
\hline Middle & $5(23 \%)$ & $6(24 \%)$ & $11(23 \%)$ \\
\hline High & $6(27 \%)$ & $3(12 \%)$ & $9(19 \%)$ \\
\hline Absence at school in the past year $-n\left(\%^{*}\right)$ & (3 missing) & (3 missing) & (6 missing) \\
\hline $0-14$ days & $14(64 \%)$ & $15(60 \%)$ & $29(62 \%)$ \\
\hline 15-30 days & $3(14 \%)$ & $1(4 \%)$ & $4(9 \%)$ \\
\hline $1-3$ months & $2(9 \%)$ & $6(24 \%)$ & $8(17 \%)$ \\
\hline $4-6$ months & $2(9 \%)$ & $1(4 \%)$ & $3(6 \%)$ \\
\hline 7-12 months & $1(4 \%)$ & $2(8 \%)$ & $3(6 \%)$ \\
\hline Functional disability (FDI, scored 0-60) - mean (SD) & $24.7(10.3)$ & $23.1(8.1)$ & 23.8(9.1) \\
\hline \multicolumn{4}{|l|}{ Pain specific quality of life (QLA CP, scored 0-3) - mean (SD) } \\
\hline Domain Psychological Functioning & $1.57(0.47)$ & $1.67(0.51)$ & $1.62(0.49)$ \\
\hline Domain Functional Status & $1.74(0.53)$ & $1.86(0.44)$ & $1.80(0.48)$ \\
\hline Domain Physical Status & $1.81(0.63)$ & $1.76(0.64)$ & $1.78(0.63)$ \\
\hline Domain Social Functioning & $1.72(0.60)$ & $1.81(0.59)$ & $1.77(0.59)$ \\
\hline Fear of Pain (FOPQ, scored 0-96) - mean (SD) & $40.1(16.7)$ & $38.7(13.7)$ & 39.3(15.0) \\
\hline Pain Catastrophizing (PCS, scored 0-52) - mean (SD) & $22.1(11.0)$ & $20.3(9.5)$ & $21.1(10.2)$ \\
\hline Depressive symptoms (CDI, scored 0-54) - mean (SD) & $26.1(2.55)$ & $25.7(2.53)$ & $25.9(2.51)$ \\
\hline Pain intensity (VAS 0-100) - mean (SD) & $53(14)$ & $55(22)$ & $54(18)$ \\
\hline $\begin{array}{l}\text { Perceived harmfulness (PHODA-Youth, } \\
\text { scored 0-510) - mean (SD) }\end{array}$ & $191(121)$ & $180(119)$ & $185(119)$ \\
\hline Credibility (CEQ, scored 3-27) - mean (SD) & $17.7(5.1)$ & $18.3(5.2)$ & $18.0(5.0)$ \\
\hline Expectancy (CEQ, scored 2-18) - mean (SD) & $13.2(2.6)$ & $12.5(3.5)$ & $12.8(3.1)$ \\
\hline
\end{tabular}

Note. ${ }^{*}$ Valid percent

** In the exposure program 19 adolescents received the standard adolescent module, and 6

adolescents received the additional physical training because of joint hypermobility syndrome.

In care as usual 4 of the 28 adolescents had joint hypermobility syndrome. 


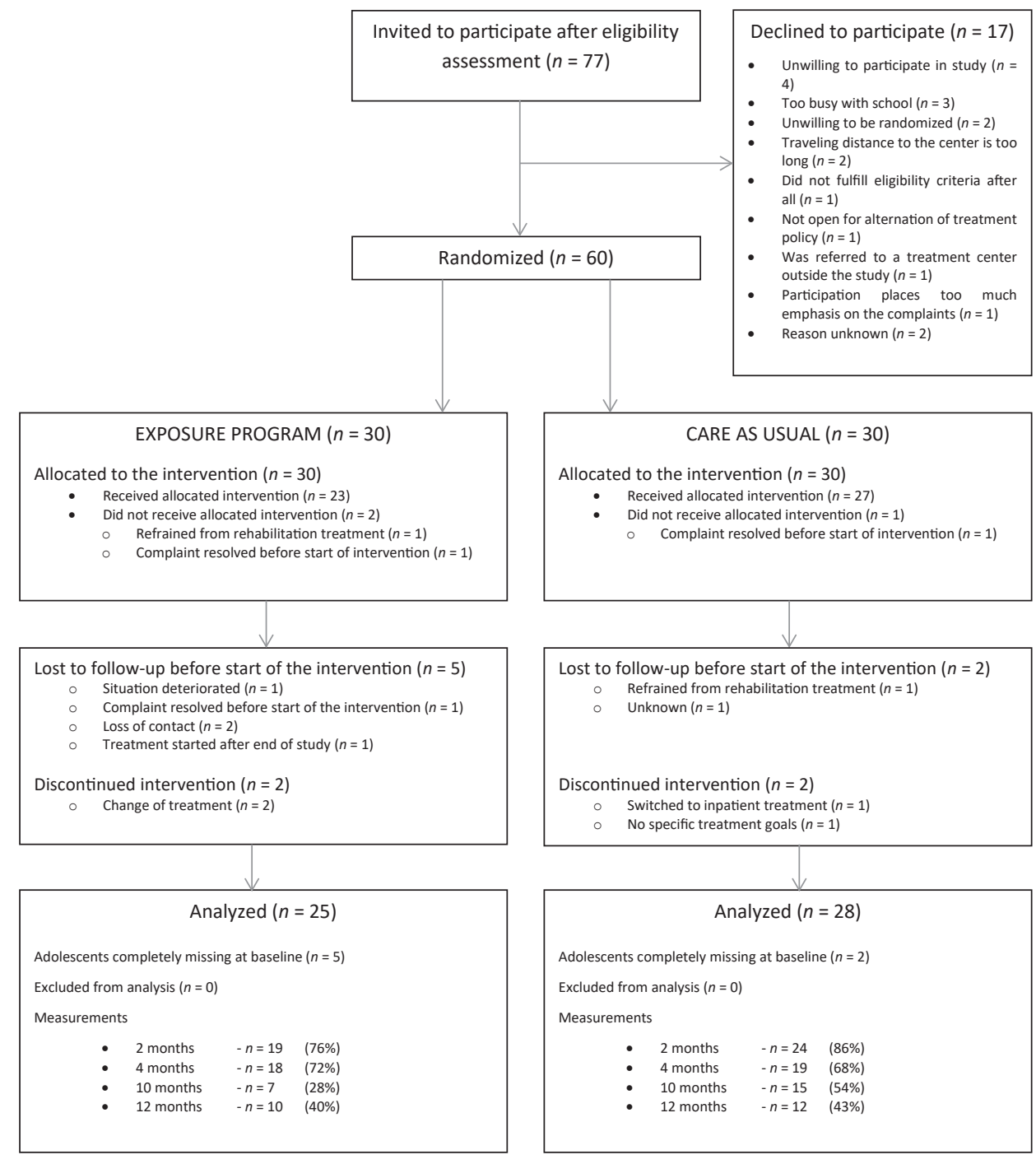

Figure 1 Adolescent flow through the RCT 


\section{Protocol adherence and contamination}

Inter-rater reliability was Cohen's kappa $=0.69$ for the assessment of the treatment elements. Protocol adherence for EP was high since on average 80.8\% (SD =11.05) of the essential treatment elements occurred (20). Contamination was on average 4.9\% (SD=9.19) in EP and 7.7\% (SD=10.30) in CAU, below the threshold and therefore absent. Overall, $92 \%$ of the recordings were classified correctly as belonging to EP or CAU. One rater misclassified one CAU recording as EP; the other rater misclassified five CAU recordings as EP.

\section{DISCUSSION}

This study demonstrated that in adolescents with chronic musculoskeletal pain reporting pain-related fear, an interdisciplinary graded exposure program led to a significantly larger decrease in functional disability than usual care at all time points. The difference of at least 8.8 FDI points that was found between the groups is statistically significant and clinically relevant (33). Additionally, the magnitude of this difference was almost twice that predicted during the design of this trial.

Considering the severity of functional disability, adolescents in EP on average improved from moderate to light/no disability. Adolescents in CAU remained, on average, in the moderately disabled category (34). Furthermore, EP was more effective in decreasing perceived harmfulness of feared and avoided activities at all time points. At some time points EP appeared more effective in reducing pain intensity, pain catastrophizing, depressive symptoms and in enhancing health-related quality of life.

The results of this trial add to the evidence on interdisciplinary chronic pain treatment to improve functional ability (e.g. (35-41)), explicitly focusing on outpatient rehabilitation treatment for adolescents with CMP. To our knowledge, this is the first RCT investigating a graded exposure program targeting pain-related fear to improve functional ability, despite pain. By taking a 12month follow-up period, the results provide insight into the treatment effects in the longer term.

With a pragmatic approach in this trial, results for the comparative effectiveness of EP and CAU are as close to routine practice as possible (42). Furthermore, eligibility criteria for referral to outpatient chronic pain rehabilitation for the trial were the same as they were for rehabilitation care outside this study. This increases the external validity of this trial. Internal validity was guaranteed by encouraging treatment teams to adhere to the EP protocol, using randomization with concealed treatment allocation, and blinding of the data collection and analysis (42).

Another strength of this trial was the evaluation of treatment delivery. Although in two of the four centers treatment teams offered either EP or CAU, in the other two centers each team offered both interventions, increasing the risk of contamination by the other intervention. Investigation showed that protocol adherence by the treatment teams in EP was high and contamination was absent, according to the pre-specified criteria. Inter-rater reliability for the rating of the treatment elements was substantial (32). Protocol adherence was only evaluated for EP. Since CAU was not offered according to the same protocol in all centers, evaluation of protocol adherence was here considered inappropriate. 


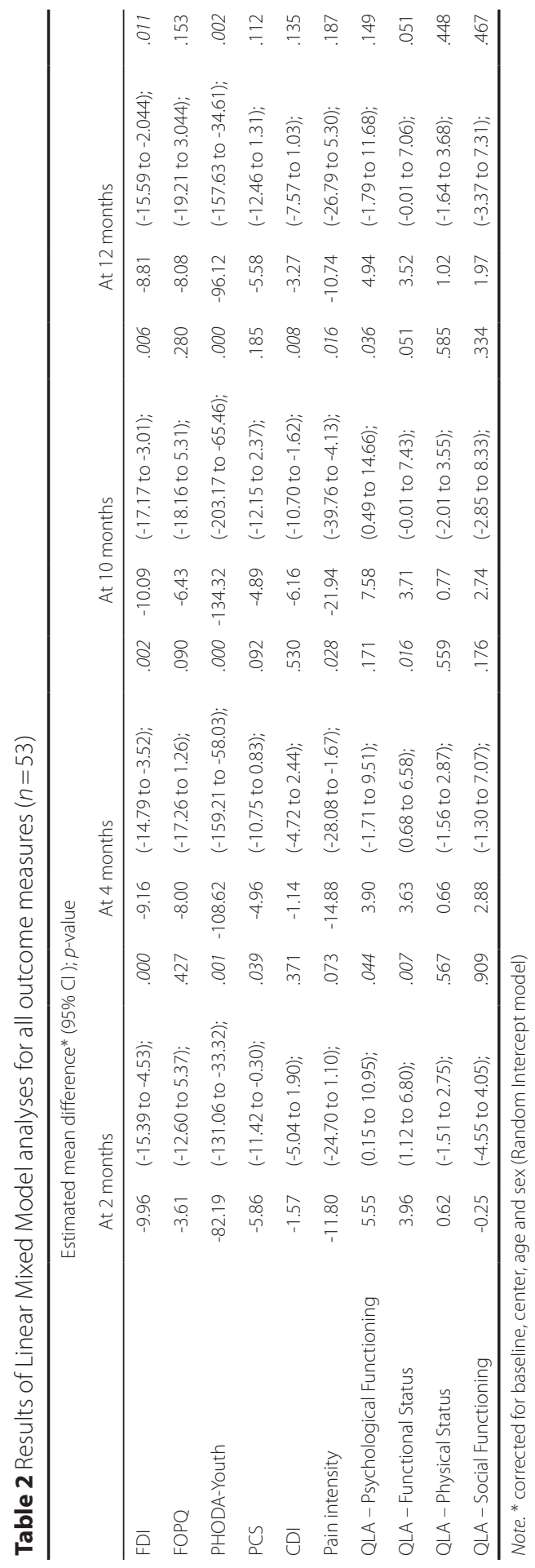




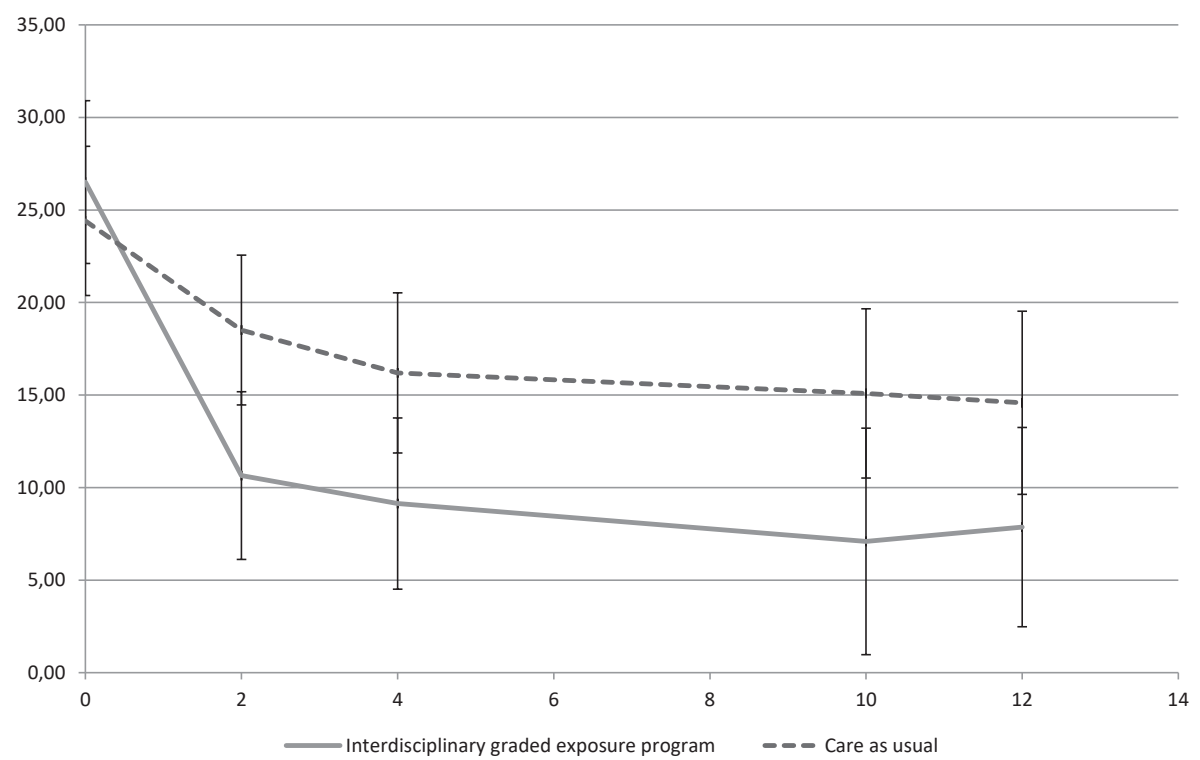

Figure 2 Estimated mean group scores on the FDI at baseline and after 2, 4, 10 and 12 months with a 95\% confidence interval.

Some limitations need attention. The specified sample size was not attained. Since the difference between the treatment groups was almost twice as large as the minimum clinically relevant difference used in the sample size calculation, the smaller than desired sample size in this trial was less of an issue. Although this lower inclusion rate did not hinder evaluation of the primary research question, for future studies this might be a point needing attention. The most important criterion for offering EP is that pain-related fear is present in the patient (9). This was not an explicit criterion in the eligibility criteria, but it was implicitly captured in the 'referral to outpatient rehabilitation'-criterion. It is, however, very important that pain-related fear is assessed during the screening. For treatment teams inexperienced in screening with a view to EP treatment, it can be a challenge to properly recognize the presence of pain-related fear. The use of the PHODA-Youth in this stage might offer a solution because this instrument was developed to identify those activities or situations perceived as harmful for the painful body part, and which are therefore feared (25). Furthermore, if pain-related fear is not identified as a (major) problem during the screening it is less appropriate to start EP. Additionally, although clinical relevant change was investigated in a population of youth with fibromyalgia, this study provides the only reference point for interpreting treatment effects on the Functional Disability Inventory currently known. Last, clinical relevant change in the FDI was studied in youth with fibromyalgia (33). To our knowledge, this is currently the only study reporting on clinical relevant change in the FDI. 


\section{CONCLUSION}

In adolescents with CMP, EP leads to a clinically relevant and significantly larger decrease in functional disability than does usual care. Furthermore, results on protocol adherence and contamination between interventions imply an honest comparison. Therefore, implementation of EP in rehabilitation care for adolescents with CMP and pain-related fear is recommended.

\section{ACKNOWLEDGMENTS}

This study was supported by a grant of Fonds Nuts Ohra, Stichting Vooruit, and Adelante Center of Expertise in Rehabilitation and Audiology.

We thank all participants in this study. We also thank the treatment teams offering both interventions, and the site coordinators assisting in the execution of study procedures of Rijndam Rehabilitation at Rotterdam, Revant Rehabilitation Center Breda, the Department of Rehabilitation Medicine of the Laurentius Hospital Roermond and Adelante Center of Expertise in Rehabilitation and Audiology Maastricht location and Valkenburg location for their participation in this trial. We thank the members of Adelante Center of Expertise in Rehabilitation and Audiology Maastricht location and the members of the Department of Rehabilitation Medicine at Maastricht University for their efforts in developing the new interdisciplinary graded exposure program. We thank J. de Jong and A. Nijhuis for training the treatment teams. We are grateful to M. de Mooij for all her efforts in offering help to all site coordinators, her work in collecting all data, informing patients and parents and all other assistance. We thank J. Zeelig and M. Geijen for their evaluations of treatment delivery. Proofreading of this manuscript was performed by scientific proofreader and editor Les Hearn. 


\section{REFERENCES}

1. Perquin CW, Hazebroek-Kampschreur AAJM, Hunfeld JAM, Bohnen AM, van Suijlekom-Smit LWA, Passchier J, et al. Pain in children and adolescents: a common experience. Pain. 2000;87(1):51-8.

2. King S, Chambers CT, Huguet A, MacNevin RC, McGrath PJ, Parker L, et al. The epidemiology of chronic pain in children and adolescents revisited: A systematic review. Pain. 2011;152(12):2729-38.

3. El-Metwally A, Salminen JJ, Auvinen A, Kautiainen H, Mikkelsson M. Prognosis of non-specific musculoskeletal pain in preadolescents: a prospective 4-year follow-up study till adolescence. Pain. 2004;110(3):550-9.

4. Fearon P, Hotopf M. Relation between headache in childhood and physical and psychiatric symptoms in adulthood: national birth cohort study. BMJ. 2001;322(7295):1145.

5. Eccleston C, Palermo TM, de CWAC, Lewandowski A, Morley S, Fisher E, et al. Psychological therapies for the management of chronic and recurrent pain in children and adolescents. Cochrane database syst rev. 2012;12:CD003968.

6. Palermo TM, Eccleston C, Lewandowski AS, Williams ACdC, Morley S. Randomized controlled trials of psychological therapies for management of chronic pain in children and adolescents: An updated meta-analytic review. Pain. 2010;148(3):387-97.

7. Hechler T, Kanstrup M, Holley AL, Simons LE, Wicksell R, Hirschfeld G, et al. Systematic Review on Intensive Interdisciplinary Pain Treatment of Children With Chronic Pain. Pediatrics. 2015;136(1):115-27.

8. de Jong JR, Vlaeyen JW, Onghena P, Cuypers C, den Hollander M, Ruijgrok J. Reduction of pain-related fear in complex regional pain syndrome type I: the application of graded exposure in vivo. Pain. 2005;116(3):264-75.

9. Leeuw M, Goossens ME, van Breukelen GJ, de Jong JR, Heuts PH, Smeets RJ, et al. Exposure in vivo versus operant graded activity in chronic low back pain patients: results of a randomized controlled trial. Pain. 2008;138(1):192-207.

10. den Hollander M, Goossens M, de Jong J, Ruijgrok J, Oosterhof J, Onghena P, et al. Expose or protect? A randomized controlled trial of exposure in vivo vs pain-contingent treatment as usual in patients with complex regional pain syndrome type 1. Pain. 2016;157(10):2318-29.

11. Vlaeyen JW, Linton SJ. Fear-avoidance and its consequences in chronic musculoskeletal pain: a state of the art. Pain. 2000;85(3):317-32.

12. Caes L, Fisher E, Clinch J, Tobias JH, Eccleston C. The role of pain-related anxiety in adolescents' disability and social impairment: ALSPAC data. Eur J Pain. 2015;19(6):842-51.

13. Simons LE, Kaczynski KJ. The Fear Avoidance model of chronic pain: examination for pediatric application. The Journal of Pain 2012;13(9):827-35.

14. Simons LE, Kaczynski KJ, Conroy C, Logan DE. Fear of pain in the context of intensive pain rehabilitation among children and adolescents with neuropathic pain: associations with treatment response. The Journal of Pain. 2012;13(12):1151-61.

15. Goubert L, Simons LE. Cognitive styles and processes in paediatric pain. In: McGrath PA, Stevens BJ, Walker SM, Zempsky WT, editors. Oxford Textbook of Paediatric Pain. Oxford: Oxford University Press; 2014. p. 95-101. 
16. Engelbert RH, Juul-Kristensen B, Pacey V, de Wandele I, Smeenk S, Woinarosky N, et al. The evidence-based rationale for physical therapy treatment of children, adolescents, and adults diagnosed with joint hypermobility syndrome/hypermobile Ehlers Danlos syndrome. Am J Med Genet C Semin Med Genet. 2017;175(1):158-67.

17. Keer R, Simmonds J. Joint protection and physical rehabilitation of the adult with hypermobility syndrome. Curr Opin Rheumatol. 2011;23(2):131-6.

18. Dekker C, Goossens ME, Bastiaenen CH, Verbunt JA. Study protocol for a multicentre randomized controlled trial on effectiveness of an outpatient multimodal rehabilitation program for adolescents with chronic musculoskeletal pain (2B Active). BMC Musculoskelet Disord. 2016;17:317.

19. Schwartz D, Lellouch J. Explanatory and pragmatic attitudes in therapeutical trials. J Clin Epidemiol. 2009;62(5):499-505.

20. Dekker C, van Haastregt JCM, Verbunt JAMCF, De Jong J, van Meulenbroek T, Pernot HFM, et al. Pain-related fear in adolescents with chronic musculoskeletal pain: Process evaluation of an interdisciplinary graded exposure program. Submitted.

21. Claar RL, Walker LS. Functional assessment of pediatric pain patients: psychometric properties of the functional disability inventory. Pain. 2006;121(1-2):77-84.

22. Walker LS, Greene JW. The functional disability inventory: measuring a neglected dimension of child health status. J Pediatr Psychol. 1991;16(1):39-58.

23. Simons LE, Sieberg CB, Carpino E, Logan D, Berde C. The Fear of Pain Questionnaire (FOPQ): Assessment of Pain-Related Fear Among Children and Adolescents With Chronic Pain. The Journal of Pain. 2011;12(6):677-86.

24. Dekker C, Bastiaenen CHG, de Vries JE, Simons LE, Goossens M, Verbunt J. Dutch version of the Fear of Pain Questionnaire for adolescents with chronic pain. Disabil Rehabil. 2017:1-7.

25. Verbunt JA, Nijhuis A, Vikstrom M, Stevens A, Haga N, de Jong J, et al. The psychometric characteristics of an assessment instrument for perceived harmfulness in adolescents with musculoskeletal pain (PHODA-youth). Eur J Pain. 2015;19(5):695-705.

26. Crombez G, Bijttebier P, Eccleston C, Mascagni T, Mertens G, Goubert L, et al. The child version of the pain catastrophizing scale (PCS-C): a preliminary validation. Pain. 2003;104(3):639-46.

27. Roelofs J, Braet C, Rood L, Timbremont B, van Vlierberghe L, Goossens L, et al. Norms and screening utility of the Dutch version of the Children's Depression Inventory in clinical and nonclinical youths. Psychol Assess. 2010;22(4):866-77.

28. Stinson JN, Kavanagh T, Yamada J, Gill N, Stevens B. Systematic review of the psychometric properties, interpretability and feasibility of self-report pain intensity measures for use in clinical trials in children and adolescents. Pain. 2006;125(1-2):143-57.

29. Merlijn VP, Hunfeld JA, van der Wouden JC, Hazebroek-Kampschreur AA, Passchier J. Shortening a quality of life questionnaire for adolescents with chronic pain and its psychometric qualities. Psychol Rep. 2002;90(3 Pt 1):753-9.

30. Leeuw M, Goossens ME, de Vet HC, Vlaeyen JW. The fidelity of treatment delivery can be assessed in treatment outcome studies: a successful illustration from behavioral medicine. J Clin Epidemiol. 2009;62(1):81-90.

31. Perepletchikova F, Kazdin A. Treatment integrity and therapeutic change: issues and research recommendations. Clin Psychol Sci Pract. 2005;12(4):365-83. 
32. Landis JR, Koch GG. The measurement of observer agreement for categorical data. Biometrics. 1977;33(1):159-74.

33. Sil S, Arnold LM, Lynch-Jordan A, Ting TV, Peugh J, Cunningham N, et al. Identifying treatment responders and predictors of improvement after cognitive-behavioral therapy for juvenile fibromyalgia. Pain. 2014;155(7):1206-12.

34. Kashikar-Zuck S, Flowers SR, Claar RL, Guite JW, Logan DE, Lynch-Jordan AM, et al. Clinical utility and validity of the Functional Disability Inventory among a multicenter sample of youth with chronic pain. Pain. 2011;152(7):1600-7.

35. Eccleston C, Malleson PN, Clinch J, Connell H, Sourbut C. Chronic pain in adolescents: evaluation of a programme of interdisciplinary cognitive behaviour therapy. Arch Dis Child. 2003;88(10):881-5.

36. Kashikar-Zuck S, Swain NF, Jones BA, Graham TB. Efficacy of cognitive-behavioral intervention for juvenile primary fibromyalgia syndrome. J Rheumatol. 2005;32(8):1594-602.

37. Hechler T, Dobe M, Kosfelder J, Damschen U, Hubner B, Blankenburg M, et al. Effectiveness of a 3-week multimodal inpatient pain treatment for adolescents suffering from chronic pain: statistical and clinical significance. Clin J Pain. 2009;25(2):156-66.

38. Hechler T, Ruhe AK, Schmidt P, Hirsch J, Wager J, Dobe M, et al. Inpatient-based intensive interdisciplinary pain treatment for highly impaired children with severe chronic pain: randomized controlled trial of efficacy and economic effects. Pain. 2014;155(1):118-28.

39. Maynard CS, Amari A, Wieczorek B, Christensen JR, Slifer KJ. Interdisciplinary behavioral rehabilitation of pediatric pain-associated disability: retrospective review of an inpatient treatment protocol. J Pediatr Psychol. 2010;35(2):128-37.

40. Simons LE, Sieberg CB, Pielech M, Conroy C, Logan DE. What does it take? Comparing intensive rehabilitation to outpatient treatment for children with significant pain-related disability. J Pediatr Psychol. 2013;38(2):213-23.

41. Logan DE, Simons LE, Carpino EA. Too sick for school? Parent influences on school functioning among children with chronic pain. Pain. 2012;153(2):437-43.

42. Patsopoulos NA. A pragmatic view on pragmatic trials. Dialogues Clin Neurosci. 2011;13(2):217-24. Epub 2011/08/17. 



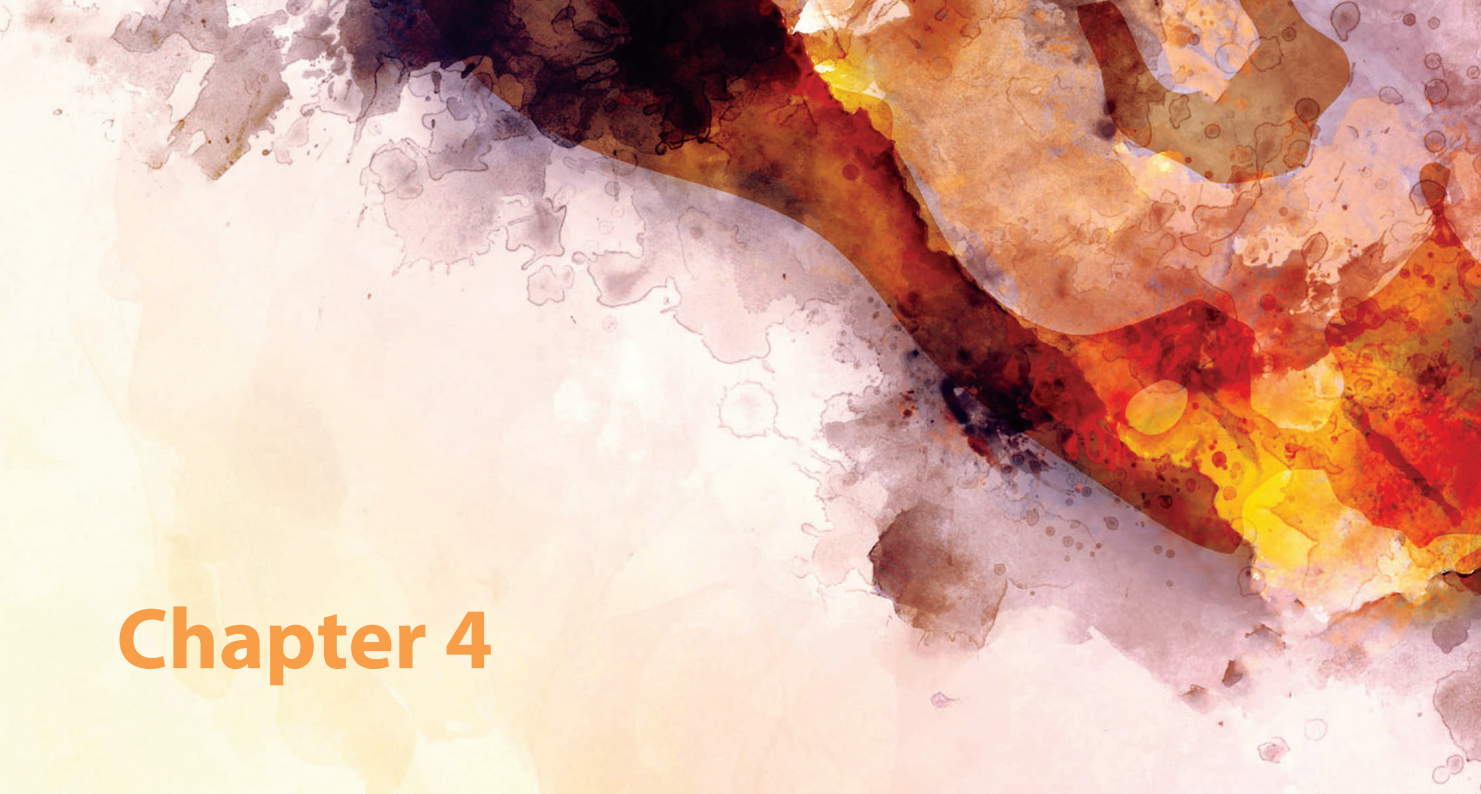

Pain-related fear in adolescents with chronic musculoskeletal pain: process evaluation of an interdisciplinary graded exposure program

Carolien Dekker

Jolanda van Haastregt

Jeanine Verbunt

Jeroen de Jong

Thijs van Meulenbroek

Dia Pernot

Agnes van Velzen

Caroline Bastiaenen

Mariëlle Goossens 


\section{ABSTRACT}

Purpose To evaluate whether an interdisciplinary graded exposure program, for adolescents with chronic musculoskeletal pain reporting pain-related fear, was performed according to protocol, and whether it is feasible to implement the program in rehabilitation care.

Methods A process evaluation where quantitative and qualitative data on participant characteristics (adolescents, parents and therapists), attendance and participants' opinion on the program were collected, by means of registration forms, questionnaires and group interviews. To evaluate treatment fidelity, audio and video recordings of program sessions were analyzed.

Results Thirty adolescents were offered the program, of which 23 started the program. Adolescents attended on average $90 \%$ of the sessions. At least one parent per adolescent participated in the program. Analysis of 20 randomly selected recordings of treatment sessions revealed that treatment fidelity was high, since $81 \%$ of essential treatment elements were offered to the adolescents. The program was considered client-centered by adolescents and family-centered by parents. Treatment teams wished to continue offering the program in their center.

Conclusion The interdisciplinary graded exposure program was performed largely according to protocol, and therapists, adolescents and their parents had a favorable opinion on the program. Implementation of the program in rehabilitation care is considered feasible.

\section{Implications for rehabilitation}

- This was the first study evaluating an interdisciplinary graded exposure program targeting pain-related fear to reduce functional disability in adolescents with chronic musculoskeletal pain.

- Treatment teams demonstrated high treatment fidelity when they offered the exposure program to the adolescents.

- Implementation of the exposure program in rehabilitation care for adolescents with chronic musculoskeletal pain is considered feasible.

\section{Keywords}

Graded exposure in vivo, Adolescent, Chronic pain, Treatment fidelity, Feasibility 


\section{INTRODUCTION}

Chronic pain in children and adolescents can substantially impact their health-related quality of life (1). The pain can interfere with physical, psychological and social functioning and can cause serious psychological distress $(1,2)$. In the long term, there is a risk that complaints continue to exist in adulthood (3-5). Because of this wide impact, chronic pain in children and adolescent is considered a complex health problem that requires interdisciplinary treatment in case of a high level of pain-related disability (6-8). Furthermore, in adolescents with chronic musculoskeletal pain, there is an elevated prevalence of joint hypermobility (9-11), for which multidisciplinary treatment is advised (12).

It has been demonstrated that pain-related fear is an important factor in explaining the severity of pain complaints and pain-related disabilities in adolescents $(13,14)$. When pain is perceived as a threat (e.g. as a sign of injury) this can provoke anticipatory fear responses and associated protective behavior. A vicious circle may develop in which catastrophizing thoughts and pain-related fear lead to avoidance of activities. Prolongation of these behaviors may have the paradoxical effect that fear, associated pain sensitivity and disability sustain (15). For the adolescent population, this mechanism is explained in the interpersonal fear avoidance model of pain (16), that incorporates the important interaction between adolescent and parents, in addition to the original fear avoidance model of Vlaeyen and Linton (17). Recently, exposure in vivo interventions showed promising results in reducing functional disability in various chronic pain conditions in adults (18-20).

Because of demonstrated effectiveness in adults and supporting evidence for the applicability of the underlying theoretical model in adolescents, an interdisciplinary graded exposure program was developed to improve adolescent functional disability by reducing pain-related fear and catastrophizing of both the adolescent and the parents. This is accomplished by challenging catastrophizing thoughts and performing feared and avoided activities in order to improve physical, psychological and social functioning. To our knowledge, graded exposure in vivo has not been studied for its effectiveness and feasibility among adolescents before.

This paper describes a process evaluation according to the framework of Saunders and colleagues (21). The objectives are to evaluate whether the exposure program was performed as intended and can be implemented in practice. The process evaluation was performed alongside a multicenter randomized controlled trial, in which the effectiveness of the newly developed exposure program compared to usual care was assessed (22). As recommended by Oakley and colleagues (23), the process evaluation was performed before the results regarding the effectiveness of the exposure program were analyzed. The assessed factors are the extent to which the exposure program was delivered according to protocol (treatment fidelity and dose delivered), the extent to which the adolescents actively participated in the exposure program (dose received - exposure) and the opinion of patients, parents and treatment teams on the exposure program (dose received - satisfaction). 


\section{MATERIALS AND METHODS}

\section{Description of the intervention}

The graded exposure program aims to restore the adolescents' age appropriate level of functioning and decrease functional disability by activating and invalidating catastrophizing thoughts about feared and avoided movements or activities. The exposure program consists of an adolescent module, and a parent module that was offered in parallel with the adolescent module. For a subgroup of adolescents the adolescent module was combined with physical training if they were diagnosed with joint hypermobility syndrome. The modules of the program are described in figure 1.

The exposure program was offered in specialized rehabilitation care, by an interdisciplinary treatment team that is led by a consultant in rehabilitation medicine, and further consisted of a psychologist, and a physiotherapist or occupational therapist (24). When an adolescent is referred to rehabilitation care, treatment started with an intake by the consultant in rehabilitation medicine and a screening. The first goal of the screening was determining which program (e.g. exposure program, or other) was most appropriate for a particular adolescent. During the intake and screening the medical history, the current medical condition and the expected capacity based on the biomedical situation of the adolescent were evaluated. The physical and psychosocial factors related to functioning, including pain-related fear were assessed. In addition, an explanation of the chronic pain syndrome was provided to the adolescent and parents. Dependent on the level of disability, the complexity of the pain problem, the level of pain-related fear, psychosocial problems, and the presence of joint hypermobility syndrome, the consultant than decided, supported by the results of the screening performed by the interdisciplinary team, whether an exposure program could be started.

Once an exposure program was started (and this will be the focus of this process evaluation), first an intake was performed by a psychologist together with a physiotherapist and/or occupational therapist. During this interdisciplinary intake, with the adolescent (and parent(s)), a biopsychosocial perspective on pain and the role of fear were further explained and were placed within the context of the family system. The Photograph Series of Daily Activities - Youth (PHODA-Youth) was used to systematically build a personalized hierarchy of activities that were feared and avoided by the adolescent (25). Subsequently, the treatment rationale was explained in an education session, and the fear avoidance model was personalized using the life story of the adolescent. Thereafter, the exposure sessions took place, in which the adolescent was exposed to feared and avoided activities during behavioral experiments, based on the personalized hierarchy. The behavioral experiments were performed to challenge catastrophizing thoughts and execute activities in order to decrease pain-related fear (17).

Adolescents with additional joint hypermobility syndrome received physical training prior to the exposure sessions, to improve their physical fitness and muscle strength $(26,27)$ (figure 1). This physical training was offered by the physical therapist, in a gym and a swimming pool. The training was part of the exposure program to ensure that physical complaints of the joint hypermobility syndrome would not hinder the execution of the feared activities. 
The parent module consisted of three meetings where parents were educated on the treatment rationale, the interpersonal fear avoidance model, the role of pain within the family system, and the parents' role in providing support and preventing relapse (figure 1).

\section{Study design}

This process evaluation used qualitative and quantitative components. Data were collected between February 2014 and February 2017, before the start of the exposure program, during the program and after the program had finished. Ethical approval for this study was provided by the Medical Ethics Committee Academic Hospital Maastricht/Maastricht University, the Netherlands, NL47323.068.13.

\section{Population}

The population who participated in this process evaluation consisted of the adolescents and their parents who attended the program, and the treatment teams who offered the program. Adolescents, 12-21 years old, were eligible for participation in the study if they were referred to outpatient rehabilitation treatment for chronic musculoskeletal pain in one of the four participating rehabilitation centers in the Netherlands (one of the centers was the department of rehabilitation medicine of a general hospital). Adolescents were excluded if there was a suspicion of a medical (orthopedic, rheumatic or neurological) disease that could fully explain the current level of severity of pain complaints, if there was a suspicion of an underlying psychiatric disease that would hamper rehabilitation treatment, or in case the adolescent was pregnant.

\section{Training of the treatment teams}

The treatment teams of three of the four participating treatment centers received a 4-day training in the exposure program and study procedures before the start of the intervention period. In one center the team was already trained in the exposure program and worked with the program protocol for two years already.

Training was conducted by experienced behavioral therapists and experts in graded exposure in vivo treatment, and members of the study team. During the training, the program modules and study procedures were explained and practiced by the treatment teams by means of role plays. During the study period, supervision was offered through telephone or email contact with the trainers. Treatment teams received a detailed treatment manual (protocol) with instructions for each program session. 

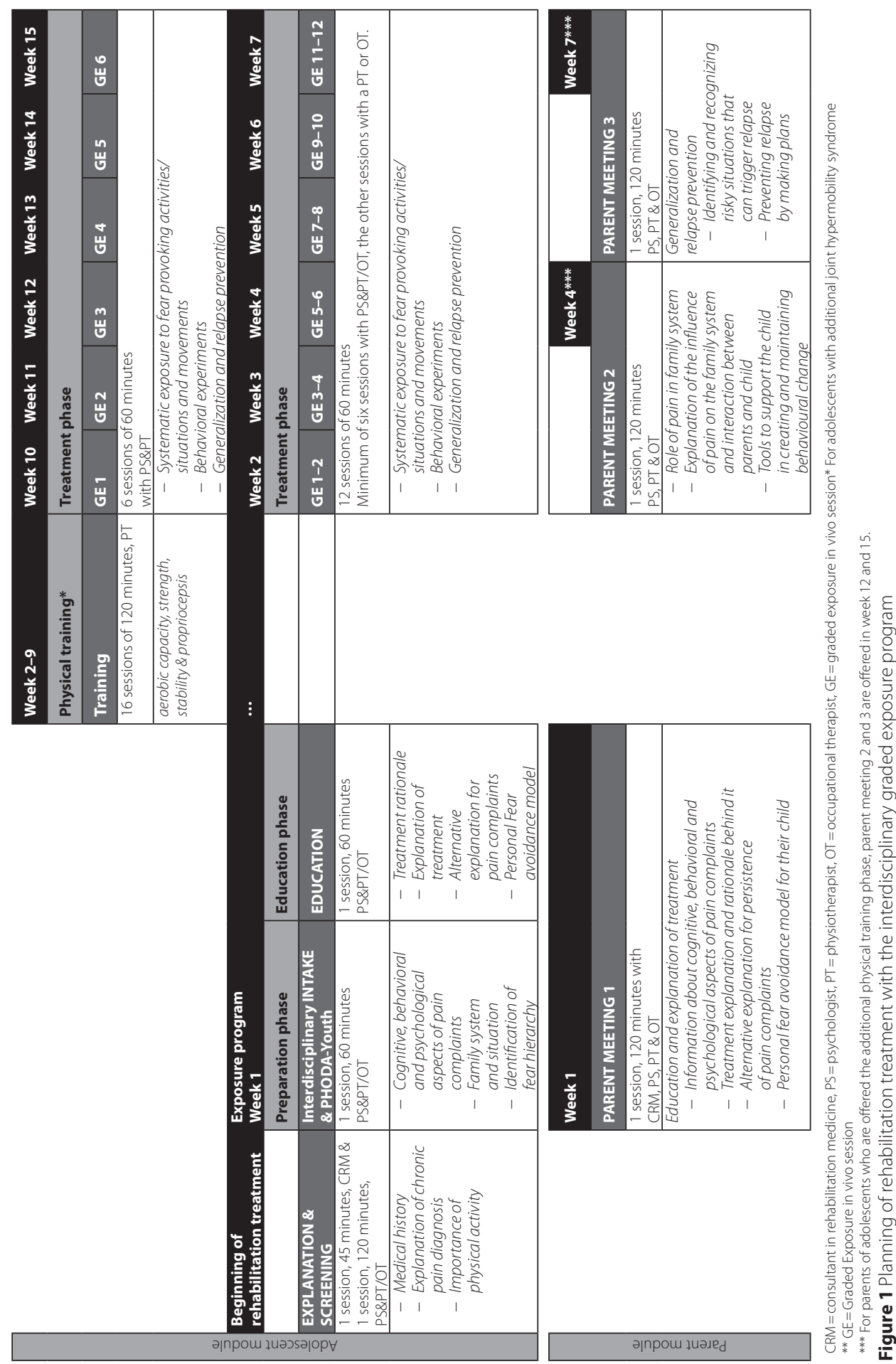


\section{Data collection}

The description of the data collection is in chronological order. Table 1 shows the components of the process evaluation, the operationalization of the components, and the data collection methods. The training of the treatment teams was evaluated by means of a questionnaire (TE), on the last training day. The questionnaire consisted of closed-end questions that could be answered on a 10-point scale ranging from 1 (totally disagree) to 10 (totally agree). Adolescents and parents received a questionnaire on participant characteristics and treatment expectancy (Q1), in the week before the start of the exposure program. An adapted version of the Credibility/Expectancy questionnaire was used to measure treatment expectancy and rationale credibility in adolescents who were about to start the exposure program $(28,29)$. Formulation of the questions was adapted to correspond with the treatment goals in the program protocol. The adapted Credibility/Expectancy questionnaire consisted of 8 questions, rated on a 9-point Likert scale ranging from 1 (not at all) to 9 (very much). Questions concern the credibility of the rationale for the treatment and treatment goals, and the improvements adolescents expect to make concerning these treatment goals. Higher scores indicate a higher credibility and higher expectations.

Performance according to protocol was divided into evaluation of treatment fidelity (the extent to which essential treatment elements were offered to the adolescent as intended), and dose delivered (the number of treatment sessions that were offered to the adolescents, and protocol deviations). Treatment fidelity was assessed for the adolescent module and was expressed as the percentage of essential treatment elements executed by the treatment teams. To enable analysis, the adolescent module was divided into phases: a preparation phase, which included the interdisciplinary intake and PHODA-youth, an education phase, which included the education session, and a treatment phase, which included the graded exposure in vivo sessions. Audio and video recordings (AV) were made by the therapists, during the three phases of the program. Specifically, the intake, education and graded exposure in vivo treatment sessions of the adolescent module were recorded. Physical training, explanation on the chronic pain syndrome, screening, and parent meetings were not recorded. Information about attendance of adolescents and parents, and protocol deviations by the treatment teams was noted on registration forms (RF).

The adolescents' opinion on the treatment they received during the program was measured with the Giving Youth A Voice questionnaire. For the parents' opinion, the Measure of Processes of Care questionnaire was used (30-33). Adolescents and their parents were asked to complete these validated questionnaires at the end of the exposure program (Q2). Both questionnaires measure client-centeredness and family-centeredness of rehabilitation services respectively. Both questionnaires consist of 20 items that can be scored on a scale of 1-7, with 7 meaning a specific action or behavior of the therapist has occurred very often, to 1 meaning this specific action or behavior has never occurred. Mean subscale scores for both the adolescent and parent questionnaire were computed. Higher ratings reflect a more favorable opinion of client- or family centeredness with the program they received. Scores were dichotomized (lowest four values in one category and highest three values in another category) to identify the lowest scoring items to indicate possibilities for program improvements. 
A group interview (Gl) was planned in each of the four treatment centers after all participants completed their exposure programs. For each meeting, the complete treatment team that offered the exposure program was invited. A topic list with predetermined open-ended questions was used as interview guide. The interview was conducted by two members of the study team, and audio recorded after consent. The evaluation focused on the performance of the program according to protocol, reasons for protocol deviations, attendance and participation of the adolescents during the program, and opinion of the therapists on the exposure program protocol they had been working with.

Table 1 Components of the process evaluation and data collection methods

\begin{tabular}{|c|c|c|c|c|c|c|}
\hline & TE & Q1 & AV & RF & Q2 & Gl \\
\hline \multicolumn{7}{|l|}{$\begin{array}{l}\text { Performance according to protocol } \\
\text { (Treatment fidelity and dose delivered) }\end{array}$} \\
\hline $\begin{array}{l}\text { Delivery of } 8 \text { essential elements of preparatory phase } \\
\text { (Elements are specified in table 2) }\end{array}$ & & & $x$ & & & \\
\hline $\begin{array}{l}\text { Delivery of } 15 \text { essential elements of education phase } \\
\text { (Elements are specified in table 2) }\end{array}$ & & & $x$ & & & \\
\hline $\begin{array}{l}\text { Delivery of } 11 \text { essential elements of treatment phase } \\
\text { (Elements are specified in table 2) }\end{array}$ & & & $x$ & & & \\
\hline Planning of the treatment sessions according to protocol & & & & $x$ & & $x$ \\
\hline \multicolumn{7}{|l|}{$\begin{array}{l}\text { Adolescent participation in the program } \\
\text { (Dose received - exposure) }\end{array}$} \\
\hline Extent to which adolescents attended the treatment sessions & & & & $x$ & & $x$ \\
\hline \multicolumn{7}{|l|}{$\begin{array}{l}\text { Opinion on the program } \\
\text { (Dose received - satisfaction) }\end{array}$} \\
\hline Opinion of the adolescents & & $x$ & & & $x$ & \\
\hline Opinion of the parents & & $x$ & & & $x$ & \\
\hline Opinion of the treatment teams & & & & & & $x$ \\
\hline Training & $x$ & & & & & \\
\hline
\end{tabular}

$T E=$ Training evaluation questionnaire, $Q 1=$ Questionnaire on characteristics of study population, $A V=$ Audio and video recordings, $R F=$ registration forms, Q2 = Questionnaire on the opinion on the program; Giving Youth A Voice Questionnaire for the adolescents and Measure of Processes of Care Questionnaire for the parents, Gl=Group interview with treatment teams

\section{Data analysis}

The quantitative data from the questionnaires and registration forms were analyzed using descriptive statistics in IBM SPSS Statistics for Windows, version 25 (IBM Corp, Armonk, NY, U.S.A.).

Treatment fidelity of the adolescent module was analyzed with an adapted version of the Method of Assessing Treatment Delivery, described by Leeuw and colleagues (34). Five steps were taken. In step 1, therapists were asked to (audio or video) record sessions in three different phases of the program. After the intervention period, the audio and video recordings were collected by the 
study team, randomly numbered, sorted per treatment center and categorized in the three program phases. For each treatment center, four sessions were randomly selected for analysis, at least one from each phase. Since one treatment center treated as much adolescents as the other three centers together, eight recordings were selected from this center. This resulted in 20 recordings that were selected for analysis. In step 2 and 3, two independent raters scored whether pre-specified treatment elements were present or absent (Table 2). The raters were a master student in developmental psychology and a health scientist. They received one day training in the treatment protocol and scoring of the three treatment phases. In step 4, inter-rater reliability between the two raters was assessed by calculating Cohen's kappa for the three phases together. In step 5 protocol adherence (treatment fidelity) was calculated. The treatment elements in the scoring forms have been scored present or absent, but these were categorized in Essential-and-Unique (EU), Essential-but-not-unique (E), Unique-but-not-essential (U), Compatible, but not essential and not unique (C) and Prohibited (P) elements for the program(34). To calculate protocol adherence, the percentage essential (EU and E) elements was calculated for each phase and for all three phases together, and should exceed 70\% for sufficient protocol adherence (34). This percentage was calculated by dividing the number of observed essential elements by the maximum possible number of these elements.

The group interviews were recorded and a transcript was made in which the answers on the open ended questions were summarized. These transcripts were coded by one of the members of the study team, to categorize the content of the answers and opinions the treatment teams expressed.

\section{RESULTS}

\section{Response and characteristics of the study population}

Between August 2014 and December 201630 adolescents were offered the exposure program, of which process data was available for 23 adolescents. For six of the 23 adolescents, parents did not complete any questionnaires because they did not consent to participate in the study $(n=5)$, or the adolescent was 18 years and decided to participate without parents $(n=1)$. Therefore, questionnaire data was available for 31 parents (14 parent couples, twice only a mother, once only a father). Seven out of the 30 adolescents and their parents did not start the program, because the complaint resolved $(n=2)$, the situation deteriorated so that outpatient treatment was no longer indicated $(n=1)$, adolescent refrained from rehabilitation treatment before start of the program $(n=1)$ or loss of contact $(n=2)$. In addition, one adolescent started after the study period. Of the 23 adolescents for whom process data was available, 18 adolescents received the standard threephase adolescent module and five adolescents also received the additional physical training.

The mean age of the adolescents was 15.6 years ( $S D=1.7$, range $12-18$ years). Twenty-two adolescents were female (96\%). Almost half of the adolescents $(48 \%, n=10)$ had a lower education, being elementary school or lower vocational education ( $n=2$ missing). For the majority of adolescents (80\%) their pain complaints started more than 1 year ago ( $n=3$ missing). Twelve adolescents (60\%, $n=3$ missing) have a relative with pain complaints. 
The participating members of the four interdisciplinary treatment teams $(n=20)$ were 4 consultants in rehabilitation medicine, 7 psychologists, 5 physiotherapists and 4 occupational therapists. For the training in the exposure program, thirteen of the sixteen (81\%) members of the treatment teams participated (one team of four members was already trained). After the intervention period, 14 (70\%) of the 20 invited therapists were present at the group interviews. Here, one physician in rehabilitation medicine, four psychologists and one occupational therapist were unable to attend.

\section{Evaluation of the training of the treatment teams}

Evaluation of the training revealed that the participants overall had a favorable opinion on the training. They considered the training relevant, the trainers competent, and they indicated that the training formed a good preparation for conducting the exposure program. However, they also made some recommendations for improving the training and preparation for the treatment. In addition to the training, they would like individual feedback on their performance with real patients to increase confidence. Therefore, they were offered the possibility to consult the trainers before and during the intervention period. However, this possibility was rarely used. Furthermore, the team members highlighted the importance of the whole team, including the consultant in rehabilitation medicine, to participate in the training (two consultants and one psychologist were unable to attend all training days, due to different circumstances). In addition, it was recommended that training should immediately be followed by the opportunity to work with adolescents in practice so that treatment teams could develop their skills. Due to practical reasons, such as the duration of the procedure for ethical approval of the study, and the set-up of the intervention and study procedures in the treatment centers, this was not immediately possible.

\section{Performance of the program according to protocol (treatment fidelity and dose delivered)}

In all centers the adolescent and parent module were offered as intended. Two centers did not offer the physical training, because no adolescents fulfilled the criteria for additional joint hypermobility syndrome. Some protocol deviations were made in all centers due to practical reasons, such as illness of a team member or absence of a swimming pool for training. Also, 12 parent modules were offered in a group setting and 11 modules were offered to individual parents/ parent couples. Reason to offer the parent module individually was due to a low number of included adolescents in two centers.

To analyze treatment fidelity, 105 of total 266 treatment sessions of the adolescent module (39\%) were recorded. According to the therapists, the main reason for not recording sessions was that it was forgotten; either to bring the recorder to the session or to turn it on. A random selection of 20 recordings (preparation phase $n=8$, education phase $n=5$, treatment phase $n=7$, in total 16 audio and four video recordings) was scored by two independent raters. Interrater reliability between the two raters was Cohen's kappa $=0.72$ for all three phases together. 
Table 2 shows the percentages of observed essential protocol elements in all three phases of the adolescent module. Treatment fidelity, scored as the mean proportion of essential treatment elements that have been offered to the adolescents, is $84 \%$ for the preparation phase, $79 \%$ for the education phase and 79\% for the treatment phase. For the three phases together, treatment fidelity was $81 \%$.

Some elements concerning parent involvement (P17, E25 and T21) score below 30\%, indicating that these elements were seldom performed during the program sessions of the adolescent. Further, elements on the aim of the program (E15 and E23), and agreements and homework (T1, T14 and T15) scored relatively low, indicating that the aim of the treatment was not clearly communicated to the adolescent during the education phase and that agreements and homework were not clearly communicated during the treatment phase. 
Table 2 Percentage of occurrences observed of the Essential* elements of the of the exposure program

\begin{tabular}{|c|c|c|c|}
\hline No. & Specific element & Category & present $^{* *}$ \\
\hline \multicolumn{4}{|c|}{ PREPARATION PHASE $-\mathrm{n}=8$ sessions evaluated } \\
\hline $\mathrm{P} 2$ & There is good teamwork between the therapists and adolescent & $\mathrm{E}$ & 100 \\
\hline P3 & The adolescent's concern/fear with regard to activities is being discussed & EU & 100 \\
\hline P6 & The adolescent assesses the level of perceived threat value of daily activities (PHODA) & EU & 94 \\
\hline P8 & The aim of the treatment session is explained to the adolescent & $\mathrm{E}$ & 88 \\
\hline P9 & The therapists respond understandingly to the problems expressed by the adolescent & $\mathrm{E}$ & 100 \\
\hline P10 & Photographs of daily activities are being used & EU & 88 \\
\hline P14 & A hierarchy is being developed based on the threat value of daily activities (PHODA) & EU & 88 \\
\hline \multirow[t]{2}{*}{ P17 } & It is discussed how parents react to the disability and pain of their child. & $\mathrm{E}$ & 13 \\
\hline & Mean proportion of essential preparation phase elements (\%) & & 84 \\
\hline \multicolumn{4}{|c|}{ EDUCATION PHASE $-\mathrm{n}=5$ sessions evaluated } \\
\hline E1 & It is emphasized that in chronic pain no clear relationship exists between pain and injury & $\mathrm{E}$ & 100 \\
\hline E3 & The adolescent's concern/fear with regard to activities is being discussed & EU & 100 \\
\hline E4 & There is good teamwork between the therapists and adolescent & $\mathrm{E}$ & 100 \\
\hline E5 & The aim of the treatment session is explained to the adolescent & $\mathrm{E}$ & 80 \\
\hline E9 & A biomechanical approach to pain is being discouraged & $\mathrm{E}$ & 70 \\
\hline E11 & It is emphasized that pain reduction is not a therapy goal & $\mathrm{E}$ & 70 \\
\hline E12 & The adolescent is being actively involved in the explanation of the therapy & $E$ & 100 \\
\hline E13 & It is explained that the treatment is aimed at verifying examining cognitions & EU & 70 \\
\hline E14 & A bio-psycho-social approach to pain is being explained & $\mathrm{E}$ & 100 \\
\hline E15 & It is emphasized that all activities are possible & $\mathrm{E}$ & 30 \\
\hline E17 & The drawbacks of inactivity are being explained & $\mathrm{E}$ & 70 \\
\hline E21 & The circular model pain-pain cognitions- avoidance - pain is being explained & EU & 90 \\
\hline E22 & The therapists respond understandingly to the problems expressed by the adolescent & $\mathrm{E}$ & 90 \\
\hline E23 & It is explained that the aim of the therapy is an increase in activity level & $\mathrm{E}$ & 40 \\
\hline \multirow[t]{2}{*}{ E25 } & The motivation of the parents for the treatment of their child is being checked & $\mathrm{E}$ & 10 \\
\hline & Mean proportion of essential education phase elements (\%) & & 79 \\
\hline \multicolumn{4}{|c|}{ TREATMENT PAHSE $-\mathrm{n}=7$ sessions evaluated } \\
\hline T1 & Homework is being assigned & E & 64 \\
\hline $\mathrm{T} 3$ & The adolescent's concern/fear with regard to activities is being discussed & EU & 100 \\
\hline $\mathrm{T} 4$ & The aim of the treatment session is explained to the adolescent & $\mathrm{E}$ & 86 \\
\hline T5 & A catastrophizing cognition is being identified & EU & 100 \\
\hline T6 & There is good teamwork between the therapists and adolescent & $\mathrm{E}$ & 100 \\
\hline T8 & A behavioral experiment is being performed & EU & 100 \\
\hline T9 & The therapists respond understandingly to the problems expressed by the adolescent & $\mathrm{E}$ & 93 \\
\hline $\mathrm{T} 11$ & Activities from the hierarchy or based on threat value are being performed & EU & 93 \\
\hline T14 & $\begin{array}{l}\text { Clear agreements are made about the way in which activities should be carried } \\
\text { out (e.g. how often, how high the jumps should be, how to bend down) }\end{array}$ & EU & 50 \\
\hline T15 & Homework is being evaluated & $\mathrm{E}$ & 44 \\
\hline \multirow[t]{2}{*}{$\mathrm{T} 21$} & It is discussed how parents react to the activities that the adolescent has started to perform. & $\mathrm{E}$ & 29 \\
\hline & Mean proportion of essential treatment phase elements (\%) & & 79 \\
\hline
\end{tabular}

*Essential treatment elements are both Essential-and-Unique (EU) and Essential-but-not-unique (E) treatment

elements (Method of Assessing Treatment Delivery, described by Leeuw and colleagues (34)).

**The reported percentage reflects the rating of both raters, therefore for each session

evaluated, two ratings were used to calculate the percentage. 


\section{Active participation in the program}

Adolescents in the standard module $(n=18)$ attended on average 12.7 of the 14 program sessions (90\%, range 8-14 sessions). Adolescents who received the standard module including physical training $(n=5)$ attended on average 21.6 of the 24 planned program sessions (90\%, range $20-24$ sessions). In the latter group 14.8 of the 16 (93\%, range 12-16) of the physical training sessions and 6.8 of the 8 ( $85 \%$, range $4-8)$ of the graded exposure sessions were attended. Of the 23 adolescents who started the program, 21 adolescents completed their program. Two adolescents did not complete their program because they changed treatment $(n=2)$. These two adolescents attended 8 of the 14 (57\%) and 20 of the 24 (83\%) planned sessions respectively.

For the parent module, attendance was retrospectively assessed in the group interview for the parents of all 23 adolescents. For all 23 adolescents, at least 1 parent attended the parent module. Approximately half of the modules were attended by both parents, the other half by one parent (for six parent couples it could not be retrieved whether one of both parents attended).

\section{Opinion of adolescents, parents and treatment teams on the program}

The adolescents' mean credibility score for the treatment rationale before the start of the exposure program was 17.7 (SD $=5.1$, on a scale of 3-27) and mean expectancy was $13.2(S D=2.6$, on a scale of $2-18$ ), scored by 20 adolescents ( $n=3$ missing). These scores indicate that adolescents assign relatively moderate credibility that the exposure program will increase their functioning and participation in social activities despite their pain. Furthermore, these scores indicate the adolescents have relatively high expectations with regard to the improvements the exposure program can cause in functioning and participation in social activities.

The Giving Youth a Voice questionnaire was completed by 17 adolescents (68\%, $n=8$ questionnaires missing), reflecting their opinion on the exposure program. Mean scores on the subscales are presented in table 3. Overall, the care these adolescents have received was scored as highly client-centered, since scores on all subscales are close to the maximum score of 7. The items 'How much do the people who work with you inform you of how treatments might harm you or help you?' and 'How much do the people who work with you offer you useful information about how you are doing?' were scored by $24 \%$ of the adolescents in the lowest category (the lowest 4 answering scores). This is where the behavior of the therapists had the lowest scores on client-centeredness. 
Table 3 Opinion about the client- and family centeredness of the program

\begin{tabular}{|c|c|c|c|}
\hline Adolescents' opinion & & Parents' opinion & \\
\hline \multirow[t]{2}{*}{$\begin{array}{l}\text { Giving Youth A Voice Questionnaire-20 subscales } \\
\text { (n items) }\end{array}$} & & $\begin{array}{l}\text { Measure of Processes of Care } \\
\text { Questionnaire-20 subscales ( } n \text { items) }\end{array}$ & \\
\hline & $\operatorname{Mean}^{*}(S D)$ & & Mean* $(S D)$ \\
\hline Supportive and Respectful Relationships (5) & $5.8(1.66)$ & Enabling and Partnership (3) & $4.7(1.63)$ \\
\hline Information Sharing/Communication (5) & $6.1(1.29)$ & Providing General Information (5) & $4.5(1.61)$ \\
\hline Supporting Independence (5) & $6.0(1.22)$ & $\begin{array}{l}\text { Providing specific information } \\
\text { about the child ( } 3 \text { ) }\end{array}$ & $5.3(1.35)$ \\
\hline \multirow[t]{2}{*}{ Teen Centered Services (5) } & $6.2(1.09)$ & $\begin{array}{l}\text { Coordinated and comprehensive } \\
\text { care for child and family (4) }\end{array}$ & $5.7(1.14)$ \\
\hline & & Respectful and supportive care (5) & $4.9(1.31)$ \\
\hline
\end{tabular}

*Mean scores on a scale 1-7, with higher scores reflecting a more favorable opinion on the behaviors of the therapists reflected in the subscales.

The Measure of Processes of Care questionnaire was completed by all participating parents $(n=31)$. Mean scores on the subscales are presented in table 3. Overall, the parents scored the care they received for their adolescent as family-centered, since mean scores on the subscales are on the higher end of the answering scale (1-7). In 8 of the 20 questions, more than $75 \%$ of the parents scored therapist behaviors to occur at least more than sometimes (highest category, highest three answering scores). The items the parents scored most in the lowest answering category (57\% and 55\%) were 'To what extent do the people who work with your child treat you as an individual rather than as a 'typical' parent of a child with a disability?', and 'To what extent do the people who work with your child have information available to you in various forms, such as a booklet, kit, video, etc.?' For these behaviors of the therapist the parents' ratings were the lowest on family-centeredness.

All participating treatment teams had a positive opinion about the exposure program, although most therapists perceived practice of the program challenging. The teams, however, intend to continue the use of the exposure program within their rehabilitation center, by integrating the program in their regular care. Multiple reasons were mentioned for the willingness to implement the exposure program. The exposure program was found to be suitable specifically for adolescents with chronic musculoskeletal pain reporting pain-related fear and avoidance behavior. According to the opinion of the treatment teams, the program could cause behavioral change in activity performance in a relatively short time frame. Furthermore, the program provided a clear outline for the adolescents in terms of planning and working towards their treatment goals, which worked well with this patient group. The parent involvement was much appreciated and considered essential to preserve treatment gains at home. However, due to the relative short duration of the intervention and the experienced relatively narrow focus of the intervention (improve functional disability by targeting pain-related fear) concerns were expressed regarding the 
long-term effects of the program. Especially with regard to relapse into previous pain behavior, and treatment of (psychological) co-morbidity, treatment teams expressed their doubts regarding treatment effectiveness. Moreover, guidance on how to handle in case of relapse in previous pain behavior was considered to be too restricted in the program protocol.

The psychologists and physiotherapists/occupational therapists also indicated they enjoyed and preferred working in a duo during the program sessions. They could support each other during program sessions; it enabled evaluation of the sessions (they felt better informed of what happened in the treatment because they were there), and it improved communication with adolescents and parents.

\section{DISCUSSION}

This process assessed whether an interdisciplinary graded exposure program offered in specialized rehabilitation care was performed according to protocol and whether it would be feasible to implement the program in rehabilitation care. Treatment fidelity was high in all three treatment phases, since on average $81 \%$ of the essential treatment elements were offered to the adolescents. Adolescent participation was high, with attendance rates of 90\% and with at least one parent participating in the parent module. Adolescents and parents considered the exposure program client-centered and family-centered respectively. Adolescents considered the program moderately credible and had relatively high expectations about attaining the treatment goals (improved functioning and participation in social activities despite pain) after their first visit to the consultant in rehabilitation medicine. Furthermore, treatment teams had a favorable opinion about the content of the exposure program, and had the intention to implement the program in regular care in their rehabilitation center. Psychologists, physiotherapists and occupational therapists appreciated working jointly together in duo's in the exposure sessions.

It is difficult to compare these results with other process evaluations of rehabilitation treatment for adolescents with chronic pain, as no studies of this kind exist to our knowledge. Leeuw and colleagues (34) provided an illustration of the application of their Method of Assessing Treatment Delivery in a rehabilitation treatment in adults with chronic low back pain. Although the aims of the studies were slightly different (Leeuw aimed to evaluate whether treatment comparisons were fair, our aims was to evaluate whether the exposure program was performed according to protocol) similar checklists to score treatment elements were used. Contrary to Leeuws study, where two interventions were compared, analysis focused in our study only on the performance of essential treatment elements in one intervention condition. Although the majority of essential elements were offered to the adolescents, this study showed that there were some elements that were not offered as planned. Elements concerning explanation of the aim of the treatment, elements concerning agreements and homework, and elements concerning parent involvement, were scored only few times. Reasons for this are unknown. However, as these elements are considered to be essential and therefore important for the high quality delivery of the intervention, performance might be enhanced by stressing these topics in the program protocol and during the training of the treatment teams. 
This study has some strengths and limitations that need to be mentioned. An important strength of this study is the analysis of treatment fidelity with the adapted Method of Assessing Treatment Delivery. Applying this method, allowed real insight into the degree to which essential protocol elements were applied by the members of the treatment teams. Together with the attendance registration, both the quantity (dose delivered), and the quality (treatment fidelity) of the adolescent module were assessed. A limitation to this approach in our study was that treatment fidelity was not evaluated for the parent module. The parent module was intended to be a group intervention and it was possible that parents who did not consent to participate in the study would be present. Therefore, it was considered not possible to record these parent meetings. Similar, treatment fidelity was not assessed for the physical training of the adolescents, so we lack insight in the fidelity of this part of the program. By all means, evaluation of treatment fidelity of all parts of the exposure program would have provided a more complete picture of the quality of delivery of the program. Furthermore, as in the approach of Leeuw and colleagues (34), our evaluation also focused on the mere assessment of treatment delivery. Treatment receipt by the patient, and enactment of the patient upon treatment were disregarded, two components that by some authors have previously been described as part of treatment fidelity as well $(35,36)$.

Another limitation is the possibility that socially desirable answers were given by the adolescents, parents and treatment teams, when asked about the exposure program. To limit this risk, adolescents and parents received a link to their questionnaires by email, which they could complete at home and results would only be published at group level. However, the group interviews with the treatment teams were performed by two members of the study team. The risk might have been lower if these meetings were led by independent interviewers, not involved in the study. Moreover, treatment teams were aware that the recordings would be used to evaluate their performance according to protocol, which may have influenced their behavior.

\section{Conclusion and recommendations}

Based on the results of this process evaluation, we conclude that the interdisciplinary exposure program has been performed largely according to protocol and that treatment fidelity of the adolescent module was high. The delivery of the exposure program was perceived client-centered by the adolescents and family-centered by the parents. Treatment teams expressed a favorable opinion about the exposure program and intend to implement the program within their rehabilitation centers. Therefore, implementation of the program is considered feasible in specialized rehabilitation care.

In case the effect evaluation of the exposure program reveals positive results on treatment effectiveness, implementation of the program in specialized rehabilitation care is recommended. However, for future research, investigation of treatment fidelity (including treatment receipt and participant enactment) of the currently not evaluated parent module and physical training is recommended, since analyzing both the complete adolescent module and parent module will provide the most complete picture of treatment fidelity of the program. 
Compared to similar graded exposure interventions in adults with chronic pain, in which a psychologist is present at each exposure session, the psychologist was allowed to be present at least every second exposure session in the treatment phase in this study. Since the members of the treatment teams expressed several advantages of working as a duo, we recommend to abide by this minimum of duo sessions when implementing the program into rehabilitation care. Further, although treatment teams were trained for four days, they perceived good practice of the program challenging. Hence, teams indicated they needed to gain real world experience and wished for feedback on their individual functioning. The possibility to contact the trainers during the intervention was rarely used. In the future, the organization of regular intervision-sessions within the centers can help to overcome this. To ensure high quality program delivery, de Jong and Verbunt (37) also emphasize the importance of continuous training, real world practice and regular team intervision meetings. Therefore, the four days of training is recommended to be a minimum and it is recommended to incorporate regular intervision-meetings and yearly refresher courses when working with the program.

\section{ACKNOWLEDGEMENTS}

We would like to thank all participants in this study for completing the questionnaires, registration forms, recordings and evaluations. We thank J. de Jong and A. Nijhuis for training the treatment teams. We thank the treatment teams of Laurentius Hospital Roermond, Rijndam Rehabilitation center in Rotterdam, Revant Rehabilitation center Breda and Adelante Center of Expertise in Rehabilitation and Audiology Maastricht location for participating in this study. We are grateful to M de Mooij for the data collection and management and preparation of the data files for analysis. Last, we want to thank J. Zeelig and M. Geijen for their efforts in rating all selected recording. We were able to work on this process evaluation because of a grant of Fonds Nuts Ohra, Stichting Vooruit and Adelante Center of Expertice in Rehabilitation and Audiology.

\section{DISCLOSURE STATEMENT}

The authors report no conflicts of interest. The authors alone are responsible for the content and writing of this article.

Data availability statement The data that support the findings of this study are available from the corresponding author, $C D$, upon reasonable request. 


\section{REFERENCES}

1. Merlijn VP, Hunfeld JA, van der Wouden JC, Hazebroek-Kampschreur AA, Passchier J, Koes BW. Factors related to the quality of life in adolescents with chronic pain. Clin J Pain. 2006;22(3):306-15.

2. Konijnenberg AY, Uiterwaal CS, Kimpen JL, van der Hoeven J, Buitelaar JK, de Graeff-Meeder ER. Children with unexplained chronic pain: substantial impairment in everyday life. Arch Dis Child. 2005;90(7):680-6.

3. El-Metwally A, Salminen JJ, Auvinen A, Kautiainen H, Mikkelsson M. Prognosis of non-specific musculoskeletal pain in preadolescents: a prospective 4-year follow-up study till adolescence. Pain. 2004;110(3):550-9.

4. Fearon P, Hotopf M. Relation between headache in childhood and physical and psychiatric symptoms in adulthood: national birth cohort study. BMJ. 2001;322(7295):1145.

5. Brattberg G. Do pain problems in young school children persist into early adulthood? A 13-year follow-up. European Journal of Pain. 2004;8(3):187-99.

6. Hechler T, Wager J, Zernikow B. Chronic pain treatment in children and adolescents: less is good, more is sometimes better. BMC Pediatr. 2014;14:262.

7. Simons LE, Kaczynski KJ. The Fear Avoidance model of chronic pain: examination for pediatric application. The Journal of Pain 2012;13(9):827-35.

8. Odell S, Logan DE. Pediatric pain management: the multidisciplinary approach. J Pain Res. 2013;6:785-90.

9. de Inocencio J. Musculoskeletal pain in primary pediatric care: analysis of 1000 consecutive general pediatric clinic visits. Pediatrics. 1998;102(6):E63.

10. Gedalia A, Press J, Klein M, Buskila D. Joint hypermobility and fibromyalgia in schoolchildren. Ann Rheum Dis. 1993;52(7):494-6.

11. Clinch J, Deere K, Sayers A, Palmer S, Riddoch C, Tobias JH, et al. Epidemiology of generalized joint laxity (hypermobility) in fourteen-year-old children from the UK: a population-based evaluation. Arthritis Rheum. 2011;63(9):2819-27.

12. Scheper MC, Engelbert RH, Rameckers EA, Verbunt J, Remvig L, Juul-Kristensen B. Children with generalised joint hypermobility and musculoskeletal complaints: state of the art on diagnostics, clinical characteristics, and treatment. Biomed Res Int. 2013;2013:121054.

13. Caes L, Fisher E, Clinch J, Tobias JH, Eccleston C. The role of pain-related anxiety in adolescents' disability and social impairment: ALSPAC data. Eur J Pain. 2015;19(6):842-51.

14. Cohen LL, Vowles KE, Eccleston C. The impact of adolescent chronic pain on functioning: disentangling the complex role of anxiety. The Journal of Pain. 2010;11(11):1039-46.

15. Vlaeyen JW, Linton SJ. Fear-avoidance model of chronic musculoskeletal pain: 12 years on. Pain. 2012;153(6):1144-7.

16. Goubert L, Simons LE. Cognitive styles and processes in paediatric pain. In: McGrath PA, Stevens BJ, Walker SM, Zempsky WT, editors. Oxford Textbook of Paediatric Pain. Oxford: Oxford University Press; 2014. p. 95-101.

17. Vlaeyen JW, Linton SJ. Fear-avoidance and its consequences in chronic musculoskeletal pain: a state of the art. Pain. 2000;85(3):317-32.

18. de Jong JR, Vlaeyen JW, Onghena P, Cuypers C, den Hollander M, Ruijgrok J. Reduction of pain-related fear in complex regional pain syndrome type I: the application of graded exposure in vivo. Pain. 2005;116(3):264-75. 
19. Leeuw M, Goossens ME, van Breukelen GJ, de Jong JR, Heuts PH, Smeets RJ, et al. Exposure in vivo versus operant graded activity in chronic low back pain patients: results of a randomized controlled trial. Pain. 2008;138(1):192-207.

20. den Hollander M, Goossens M, de Jong J, Ruijgrok J, Oosterhof J, Onghena P, et al. Expose or protect? A randomized controlled trial of exposure in vivo vs pain-contingent treatment as usual in patients with complex regional pain syndrome type 1. Pain. 2016;157(10):2318-29.

21. Saunders RP, Evans MH, Joshi P. Developing a process-evaluation plan for assessing health promotion program implementation: a how-to guide. Health Promot Pract. 2005;6(2):134-47.

22. Dekker C, Goossens ME, Bastiaenen CH, Verbunt JA. Study protocol for a multicentre randomized controlled trial on effectiveness of an outpatient multimodal rehabilitation program for adolescents with chronic musculoskeletal pain (2B Active). BMC Musculoskelet Disord. 2016;17:317.

23. Oakley A, Strange V, Bonell C, Allen E, Stephenson J. Process evaluation in randomised controlled trials of complex interventions. BMJ. 2006;332(7538):413-6.

24. Spek EM, Menxel DAJV, Meulenbroek TV, Verbunt JA. Intake revalidatiearts en screening [Intake interview by the consultant in rehabilitation medicine and screening]. In: Verbunt JA, Smeets RJEM, editors. Graded Exposure een cognitief gedragsmatige aanpak van chronische pijn. Houten: Bohn Stafleu van Loghum; 2017.

25. Verbunt JA, Nijhuis A, Vikstrom M, Stevens A, Haga N, de Jong J, et al. The psychometric characteristics of an assessment instrument for perceived harmfulness in adolescents with musculoskeletal pain (PHODA-youth). Eur J Pain. 2015;19(5):695-705.

26. Engelbert RH, Juul-Kristensen B, Pacey V, de Wandele I, Smeenk S, Woinarosky N, et al. The evidence-based rationale for physical therapy treatment of children, adolescents, and adults diagnosed with joint hypermobility syndrome/hypermobile Ehlers Danlos syndrome. Am J Med Genet C Semin Med Genet. 2017;175(1):158-67.

27. Keer R, Simmonds J. Joint protection and physical rehabilitation of the adult with hypermobility syndrome. Curr Opin Rheumatol. 2011;23(2):131-6.

28. Devilly GJ, Borkovec TD. Psychometric properties of the credibility/expectancy questionnaire. J Behav Ther Exp Psychiatry. 2000;31(2):73-86.

29. Goossens ME, Vlaeyen JW, Hidding A, Kole-Snijders A, Evers SM. Treatment expectancy affects the outcome of cognitive-behavioral interventions in chronic pain. Clin J Pain. 2005;21(1):18-26; discussion $69-72$.

30. Siebes RC, Maassen GH, Wijnroks L, Ketelaar M, van Schie PE, Gorter JW, et al. Quality of paediatric rehabilitation from the parent perspective: validation of the short Measure of Processes of Care (MPOC-20) in the Netherlands. Clin Rehabil. 2007;21(1):62-72.

31. Siebes RC, Wijnroks L, Ketelaar M, van Schie PE, Vermeer A, Gorter JW. Validation of the Dutch Giving Youth a Voice Questionnaire (GYV-20): a measure of the client-centredness of rehabilitation services from an adolescent perspective. Disabil Rehabil. 2007;29(5):373-80.

32. King S, King G, Rosenbaum P. Evaluating health service delivery to children with chronic conditions and their families: Development of a refined measure of processes of care (MPOC-20). Child Health Care. 2004;33(1):35-57.

33. Gan C, Campbell KA, Snider A, Cohen S, Hubbard J. Giving Youth a Voice (GYV): a measure of youths' perceptions of the client-centredness of rehabilitation services. Can J Occup Ther. 2008;75(2):96-104. 
34. Leeuw M, Goossens ME, de Vet HC, Vlaeyen JW. The fidelity of treatment delivery can be assessed in treatment outcome studies: a successful illustration from behavioral medicine. J Clin Epidemiol. 2009;62(1):81-90.

35. Bellg AJ, Borrelli B, Resnick B, Hecht J, Minicucci DS, Ory M, et al. Enhancing treatment fidelity in health behavior change studies: best practices and recommendations from the NIH Behavior Change Consortium. Health Psychol. 2004;23(5):443-51.

36. Lichstein KL, Riedel BW, Grieve R. Fair tests of clinical trials: A treatment implementation model. Advances in Behaviour Research and Therapy. 1994;16(1):1-29.

37. Jong JRd, Verbunt JA. Toepassen van graded exposure vergt training en oefening, ook voor de behandelaar [Practice of graded exposure requires training and practice, for therapists as well] In: Verbunt JA, Smeets RJEM, editors. Graded Exposure een cognitief gedragsmatige aanpak van chronische pijn. Houten: Bohn Stafleu van Loghum; 2017. 


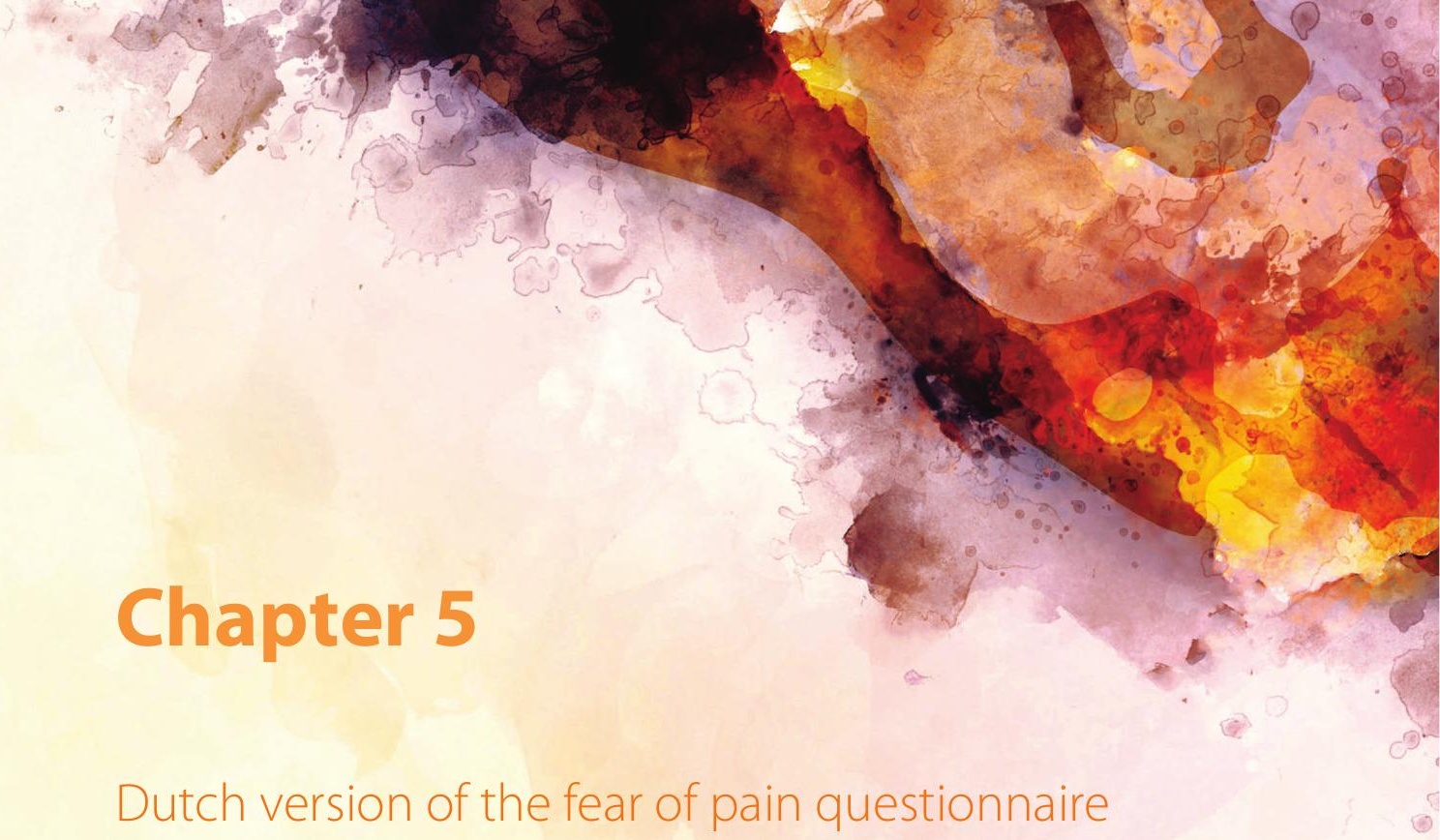

for adolescents with chronic pain

Carolien Dekker

Caroline Bastiaenen

Janneke de Vries

Laura Simons

Mariëlle Goossens

Jeanine Verbunt

Disability and Rehabilitation (2017) DOI 10.1080/09638288.2017.1289255 


\section{ABSTRACT}

Background Fear of pain is important in the development and maintenance of chronic pain. The Fear of Pain Questionnaire - Child version has been developed to assess pain related fear in children and adolescents.

Objective Translating the original questionnaire into Dutch, and investigating internal consistency and construct validity to enable use in the Dutch pain rehabilitation setting for treatment and research.

Methods Cross-sectional validation study: After forward and back translation of the FOPQ-C, adolescents (11-22 years old) with chronic musculoskeletal pain completed an assessment containing the Dutch Fear of Pain Questionnaire, and questionnaires about demographics, pain catastrophizing, functional disability and pain intensity. Internal consistency and construct validity were evaluated through exploratory factor analysis (principal axis factoring with oblique rotation) and hypotheses testing using pain catastrophizing, functional disability, and pain intensity as comparative constructs.

Results Eighty-six adolescents completed the assessment. Exploratory factor analysis resulted in a two-factor structure, explaining $43 \%$ of the variance. Internal consistency was strong (Cronbach's $a=.92$ total scale, $a=0.88$ factor 1 , and $a=.86$ factor 2 ). Five out of 6 hypotheses were confirmed.

Conclusion The Dutch version demonstrated good internal consistency and good construct validity in a population of adolescents with chronic musculoskeletal pain.

\section{Keywords}

Fear of Pain, Avoidance of Activities, Adolescent, Chronic Pain, Construct validity, Exploratory Factor Analysis 


\section{INTRODUCTION}

Chronic musculoskeletal pain (CMP) in children and adolescents is a common problem that affects approximately $4-40 \%$ of children and adolescents, depending on differences in definition of various types of pain and methodology (1). Pain can be described as chronic if it last for 3 months or longer. Recognition of the problem is growing. Consequently, there is a growing need for measures to diagnose and evaluate CMP, and related aspects such as pain related fear, within the context of the daily life of these young people. There is an urgency to gain insight into, and to optimize psychometric abilities of these measures. This may benefit future care and research (2), for example because measurement instruments in different languages can enable comparison between countries.

Chronic pain is often approached from a biopsychosocial perspective. Pain related fear is repeatedly described in relation to chronic pain $(3,4)$.'Pain related fear emerges when stimuli that are related to pain are perceived as threatening'(5). It is a complex construct that may involve fear of re-injury, fear of movement, physical activity or a combination of these factors (5). Oftentimes, patients do not report feeling fearful of pain, but simply state that it is difficult to perform required activities or movements. According to the interpersonal fear avoidance model (IFAM), both fear of pain/movement and catastrophic thinking play an important role in the development and maintenance of chronic pain problems $(4,6)$.

The Fear of Pain Questionnaire child version (FOPQ-C) was developed to assess pain related fear in children and adolescents with chronic pain. The FOPQ-C original measure demonstrates sound psychometric properties (5). To enable use of the FOPQ-C in Dutch adolescents with CMP, translation is necessary. The aim of this study, after forward and back translation of the FOPQ-C into Dutch, was to investigate construct validity by means of exploratory factor analysis and consequent hypothesis testing. Furthermore, internal consistency of the total scale and possible subscales were evaluated.

\section{METHODS}

\section{Participants \& Procedures}

This study was designed as a cross-sectional validation study. The data for the validation study were collected by the Department of Rehabilitation Medicine at Maastricht University Medical Center, the Netherlands, between January 2013 and July 2014. In total, 105 adolescents (age 11-22 years old) visiting the consultant of rehabilitation medicine seeking help for chronic musculoskeletal pain complaints were invited to participate in the study.

After initial intake by a consultant in rehabilitation medicine, the adolescents were invited to complete a screening questionnaire set. For the purpose of the validation study, the translated FOPQ-C was added to the screening questionnaire set. The goal of the screening is to collect information about the level of functioning and the impact of pain in the adolescent's life and to 
identify facilitating and disabling factors for changing the current situation with pain. The screening questionnaire was sent to the participant by providing a link in an email and personal log-in code, allowing the adolescent to complete the questionnaire at home.

For eligibility, chronic musculoskeletal pain was defined as having musculoskeletal pain complaints for 3 months or longer. Furthermore, no specific somatic (rheumatoid, neurological and orthopedic) conditions could be diagnosed as the cause or severity of the current pain complaints. A consultant in rehabilitation medicine evaluated these criteria. The Medical Ethical Committee azM/UM approved of the study (NL41712.068.12/METC 12-3-052), and the consent procedure. Patients consented for the use of the data for scientific research.

\section{Measures}

All measurement instruments used for this study are self-reported questionnaires, completed by the participants of the validation study.

\section{Pain related fear}

The FOPQ-C assesses pain related fear and avoidance in children and adolescents with chronic pain (5). The USA version (original version) of the FOPQ-C consists of 24 items, each scored on a 5-point Likert scale, ranging from 0-4 (strongly disagree to strongly agree). Total FOPQ-scores can be calculated by summing scores of all individual items. Total scores range from 0-96, with a higher score indicating more fear and avoidance. In the original child version, two subscales can be distinguished: 'fear of pain' (13 items, Cronbach's $a=89$ ) and 'avoidance of activities' (11 items, Cronbach's $a=.86$ ) (5). The fear of pain subdomain seems to encompass the cognitive and physiological aspects of pain related fear. Avoidance of activities refers to behavior aimed at postponing or preventing activities that are assumed to increase pain or (re)injury (5), thereby emphasizing behavioral aspects of pain related fear. The FOPQ-C demonstrated strong internal consistency (Cronbach's a of the total scale $=.92$ ) and moderate stability, as well as good construct validity with moderate to high correlation between FOPQ-C and measures of somatic symptoms, anxiety symptoms and catastrophizing (r ranged between .52-.70) (5).

\section{Translation procedure}

A Dutch version of the FOPQ-C was derived by systematically translating the English questions into Dutch by the first translators ( $J V$ and MG). The translation was checked for understandability by Flemish adolescents (LG). Thereafter, a native English speaker (DC) translated the Dutch questionnaire back into the English language. The author of the original version (LS) was involved in the translation procedure. 


\section{Catastrophizing}

The Pain Catastrophizing Scale Child (PCS-C, Dutch version) was used to assess negative thinking associated with pain (7). The PCS consists of 13 items describing different thoughts and feelings that children may experience when they are in pain, divided over three dimensions Rumination, Magnification and Helplessness. All items are rated on a 5-point Likert scale ranging from 0 (not at all) to 4 (a lot). Higher scores indicate higher levels of catastrophic thinking. The PCS-C demonstrated high internal consistency (Cronbach's a ranges between .68-.87 for the total scale and subscales in a community sample and .90 in a clinical sample). Preliminary investigation of construct and predictive validity is promising with correlations of $r=.49$ with pain intensity and $r=.50$ with functional disability (7).

\section{Functional disability.}

The Functional Disability Inventory (FDI) for adolescents measures perceived difficulty in performing activities in the domains of school, home, recreation and social interaction. The FDI has 15 items that are rated on a 5-point Likert scale ranging from 0 (no trouble) tot 4 (impossible) (8). Internal consistency was demonstrated to be high (Cronbach's $a=.91$ for girls and .86 for boys), as well as test-retest reliability ( $r=.74$ for two-week, and $r=.48$ for three month), in patients aged $8-17$ years with chronic abdominal pain. On construct validity correlations with somatic symptoms ( $r=.59)$ and depressive symptoms $(r=.52)$ have been reported (9). Although translated and used in Dutch population, no proper (cross-cultural) validation study on the FDI in Dutch language could be found (10).

\section{Pain intensity}

Pain intensity was measured by a $10 \mathrm{~cm}$ visual analogue scale (VAS) with the end points 'no pain' and 'the worst pain I can imagine.' For the intensity score, the average score was taken of three VAS-scales asking about the current level of pain, the worst pain in the past week and the least pain in the past week. The pain severity VAS has good reliability and validity among individuals as young as 9 year (11).

\section{Data analysis}

Analyses were performed using SPSS version 23 computer software. The digital assessment alerted participants if questions were left unanswered, which minimized missing answers. Missing items were not replaced. Descriptive statistics were used to examine participant characteristics such as gender, age, educational level, school participation and absenteeism, and pain related characteristics of the participant sample (table 1). 
Analysis of factor structure and internal consistency

After translation, the Dutch version of the FOPQ-C was approached as a 'new' instrument and exploratory factor analysis (EFA) was used to determine factor structure, with the goal of describing underlying dimensions of the variables (12). All 24 items of the FOPQ-C were investigated for normality of the scoring distributions. Intercorrelations between the items were investigated for a lack of correlation and multicollinearity (inter-item correlation . $2<r<.9$ ) in the correlation matrix (13). Corrected item-total correlations for all FOPQ-C items were calculated. Principal Axis Factoring was performed with 24 items of the FOPQ-C. To determine the number of factors to retain Kaiser's Eigenvalue $>1$ rule, interpretation of the Scree plot were used. A 1-, 2- and 3-factor structure on meaningfulness of the factors was investigated. Items with loadings $>.4$ were automatically retained and other items loading $<.4$ were individually inspected for clinical relevance. Factor structure was established by the use of oblique promax rotation. Internal consistency was evaluated by the use of bivariate Pearson correlations for both subscales and the total scale, and Cronbach's a was calculated. Values of Cronbach's a between 0.70 and 0.95 were considered good (14).

\section{Conceptual framework and hypotheses for evaluation of construct validity}

To assess construct validity, the scores of the FOPQ-C were correlated with scores of theoretically more or less related measures, being the Pain Catastrophizing Scale Child version (PCS-C), the Functional Disability Inventory (FDI) and pain intensity measured by a Visual Analogue Scale. Pain catastrophizing (PCS-C) is a construct closely related to pain related fear, but differs from pain related fear $(15,16)$. Where pain catastrophizing encompasses a cognitive response of worrying, amplifying and feeling helpless about a pain experience (7), pain related fear is a broader construct that comprises cognitive, behavioral and physiological elements (17). In line with previous research $(5,15,16)$, a moderate to high positive correlation $(0.5<r \leq 0.9)$ between the PCS-C and the FOPQ-C total scales and subscales was hypothesized. Functional disability (FDI) is related to pain related fear in a way that pain related fear could lead to functional disability according to the IFAM. Several studies have reported on this relationship $(5,16,18,19)$. Based on these studies, a low positive $(0.3<r \leq 0.5)$ correlation between the FOPQ and FDI was hypothesized. For the relationship between Pain intensity, measured with a Visual Analogue Scale (VAS), and pain-related fear a very low but positive correlation $(0.1<r \leq 0.3)$ was hypothesized $(4,5)$. The strengths of these hypothesized correlations are based on the theoretical construct definitions, empirical data of the original validation study and related research. The relationships between the constructs and expected strengths of the correlations are displayed in table 2. 
Table 2 Hypothesized strengths of correlations between FOPQ-C and construct variables

\begin{tabular}{lccccc}
\hline & $\begin{array}{c}\text { FOPQ-C Fear } \\
\text { of pain }\end{array}$ & $\begin{array}{c}\text { FOPQ-C } \\
\text { Avoidance of } \\
\text { activities }\end{array}$ & PCS-C total & FDI & Pain intensity \\
FOPQ-C total & - & - & $0.7<r \leq 0.9$ & $0.3<r \leq 0.5$ & $0.1<r \leq 0.3$ \\
FOPQ-C Fear of pain & - & $0.5<r \leq 0.7$ & $0.7<r \leq 0.9$ & - & - \\
$\begin{array}{l}\text { FOPQ-C Avoidance } \\
\text { of activities }\end{array}$ & $0.5<r \leq 0.7$ & - & $0.5<r \leq 0.7$ & - \\
\hline
\end{tabular}

Analysis of construct validity

To investigate construct validity by means of hypothesis testing, Pearson correlations were calculated between the FOPQ-C total scale and subscales (factors) with the PCS-C total, FDI, and Pain Intensity. In order to evaluate construct validity as good, at least $75 \%$ of our hypotheses should be confirmed (14).

\section{RESULTS}

\section{Sociodemographic variables and pain related variables}

Characteristics of the study population are presented in table 1. Nineteen participants were excluded from the analysis, because they completed none of the questionnaires in the assessment. Scores of eighty-six participants (76 girls) with a mean age of 16 years were included in the analysis. All participants had chronic musculoskeletal pain with varying diagnoses. In $52 \%$ of the participants, the current pain complaints started between 1 and 5 years ago. Table 3 reports on the average scores on all four measurements used in this study. 
Table 1 Characteristics of the study population $(n=86)$

\begin{tabular}{|c|c|}
\hline Age (mean, SD, range) & $16(2.6)(11-22)$ \\
\hline Gender ( $n, \%$ female) & $76(88 \%)$ \\
\hline \multicolumn{2}{|l|}{ Educational level (n, \%) } \\
\hline Elementary school & $8(9.3 \%)$ \\
\hline Lower general secondary education & $14(16.3 \%)$ \\
\hline Higher general secondary education & $20(23.3 \%)$ \\
\hline Pre university education & $17(19.8 \%)$ \\
\hline Vocational education & $20(23.3 \%)$ \\
\hline University of applied sciences & $6(7 \%)$ \\
\hline University & $1(1.2 \%)$ \\
\hline \multicolumn{2}{|l|}{ School absences in the past year $(n, \%)$} \\
\hline 0-14 days & $37(43 \%)$ \\
\hline $15-30$ days & $14(16.3 \%)$ \\
\hline $1-3$ months & $18(20.9 \%)$ \\
\hline$>3$ months & $15(17.4 \%)$ \\
\hline Missing & $2(2.3 \%)$ \\
\hline \multicolumn{2}{|l|}{ Pain related characteristics } \\
\hline \multicolumn{2}{|l|}{ Onset of current pain complaints ( $\mathrm{n}, \%)$} \\
\hline$<3$ months ago & $2(2.4 \%)$ \\
\hline $3-6$ months ago & $4(4.7 \%)$ \\
\hline $6-12$ months ago & $19(22.1 \%)$ \\
\hline $1-5$ years ago & $45(52.3 \%)$ \\
\hline$<5$ years ago & $10(11.6 \%)$ \\
\hline Missing & $6(7 \%)$ \\
\hline \multicolumn{2}{|l|}{ Type of pain problem $(n, \%)$} \\
\hline $\begin{array}{l}\text { Generalized pain problem/Fibromyalgial } \\
\text { hypermobility syndrome }\end{array}$ & $28(32.6 \%)$ \\
\hline Complex Regional Pain Syndrome & $10(11.6 \%)$ \\
\hline Chronic back pain/Neck pain/Whiplash & $22(25.6 \%)$ \\
\hline Pain in upper extremities & $6(7 \%)$ \\
\hline Pain in lower extremities & $17(19.8 \%)$ \\
\hline Pain in other specified regions & $3(3.5 \%)$ \\
\hline $\begin{array}{l}\text { Parent or other significant person with } \\
\text { chronic pain complaints }(n, \%)\end{array}$ & $28(32.6 \%)$ \\
\hline
\end{tabular}


Table 3 Descriptive statistics of all study variables

\begin{tabular}{lcccc}
\hline & Mean & SD & Range & $\mathrm{n}$ \\
\hline FOPQ-C Total (Dutch version) & 37.65 & 16.05 & $0-71$ & 86 \\
FOPQ-C Fear (Dutch version, 15 items) & 23.03 & 10.49 & $0-46$ & 86 \\
FOPQ-C Avoidance (Dutch version, 9 items) & 14.62 & 7.18 & $0-28$ & 86 \\
PCS-C Total & 20.53 & 10.63 & $1-43$ & 86 \\
PCS-C Rumination & 7.78 & 3.94 & $0-16$ & 86 \\
PCS-C Magnification & 2.34 & 2.16 & $0-9$ & 86 \\
PCS-C Helplessness & 10.42 & 5.68 & $0-23$ & 86 \\
FDI & 23.10 & 9.43 & $2-44$ & 79 \\
Pain intensity & 60.22 & 19.83 & $4-100$ & 86 \\
\hline
\end{tabular}

aPain Intensity, average rating of current pain, worst pain in the past week and least pain in the last week measured on a $10 \mathrm{~cm}$ visual analogue scale.

Normality of the scoring of the FOPQ-C items was assumed after visual inspection of the data and investigation of skewness (range -.61 to 1.07) and kurtosis (range -1.23 to .45). Furthermore, in all 24 FOPQ-C-items, except for item 5, the whole range of the answering scale (0-4) was used. On average, $24 \%$ (range $7 \%-43 \%$ ) of the participants scored in the lowest answering category of the Likert scale. Cumulative, $51 \%$ of the participants scored on the lowest two answering categories, meaning that they either disagreed or strongly disagreed with the statement about fear made in the items. The highest answering category was answered by on average $7 \%$ out of 23 items (range 1\%-22\%).

Item-total correlations ranged between 0.34 and 0.72 ; hence no items were excluded at this stage. Correlations $>0.9$ were absent and multicollinearity was ruled out. Many inter-item correlations were $<0.3$, but all items correlated with at least 1 other item. All 24 translated items were entered into an exploratory factor analysis, using Principal axis factoring as extraction method with oblique promax rotation. Results are shown in table 4. Kaiser-Meyer-Olkin measure of sampling adequacy (KMO) was 0.82 and Bartlett's Test was significant $(p<.000)$. Based on Cattel's Elbow criterion a two-factor structure was identified, explaining $43 \%$ of the variance. Four items (item 12, 17, 23 and 24) had loadings <0.4, however, on clinical relevance (item 12 and 17) and difficult formulation of the items (items 23 and 24) these items were kept in the analysis. This resulted in a 24 item Dutch version of the FOPQ-C, containing 15 items in Factor 1, which could be labeled as 'Fear of Pain' subscale, and 9 items in Factor 2, which could be labeled as 'Avoidance of Activities' subscale.

Internal consistency was then calculated, resulting in a Cronbach's a of 0.91 for the total scale (24 items), Cronbach's a of 0.88 for the 'Fear of Pain' subscale (Factor 1) and Cronbach's a of 0.86 for the 'Avoidance of Activities' subscale (Factor 2). Deletion of any one item did not significantly improve the internal consistency of the subscales. These results are shown in table 4. 
Cronbach's a did not change for the 'Fear of Pain' subscale (Factor 1), with or without the added items 12, 23 and 24 with loadings <.4. Cronbach's a of the 'Avoidance of Activities'subscale (Factor 2) did not change either with or without item 17 added.

\section{Hypotheses testing for construct validity}

The results of all calculated correlations for testing the hypotheses about construct validity are presented in table 5. All construct variables were positively correlated with higher levels of pain-related fear. The FOPQ-C total scale and subscales correlated highest with the other construct closest in the IFAM: pain catastrophizing measured with the PCS-C. The FOPQ-C total score correlated highly with pain catastrophizing (PCS-C total score; $r=.76, p<.01$ ). The FOPQ-C fear of pain subscale correlated highly with catastrophizing (PCS-C total score; $r=.78, p<.01$ ), and the FOPQ-C Avoidance of activities subscale correlated moderately with catastrophizing (PCS-C total score; $r=.56, p<.01$ ). The correlation between FOPQ-C total scale and functional disability was higher than hypothesized ( $F D I ; r=.58, p<.01$ ). As hypothesized, the correlation between fear of pain and pain intensity was very low $(r=.14, p>.05)$. Both subscales of the FOPQ-C correlated moderately with each other $(r=.64, p<.01)$, as hypothesized. 


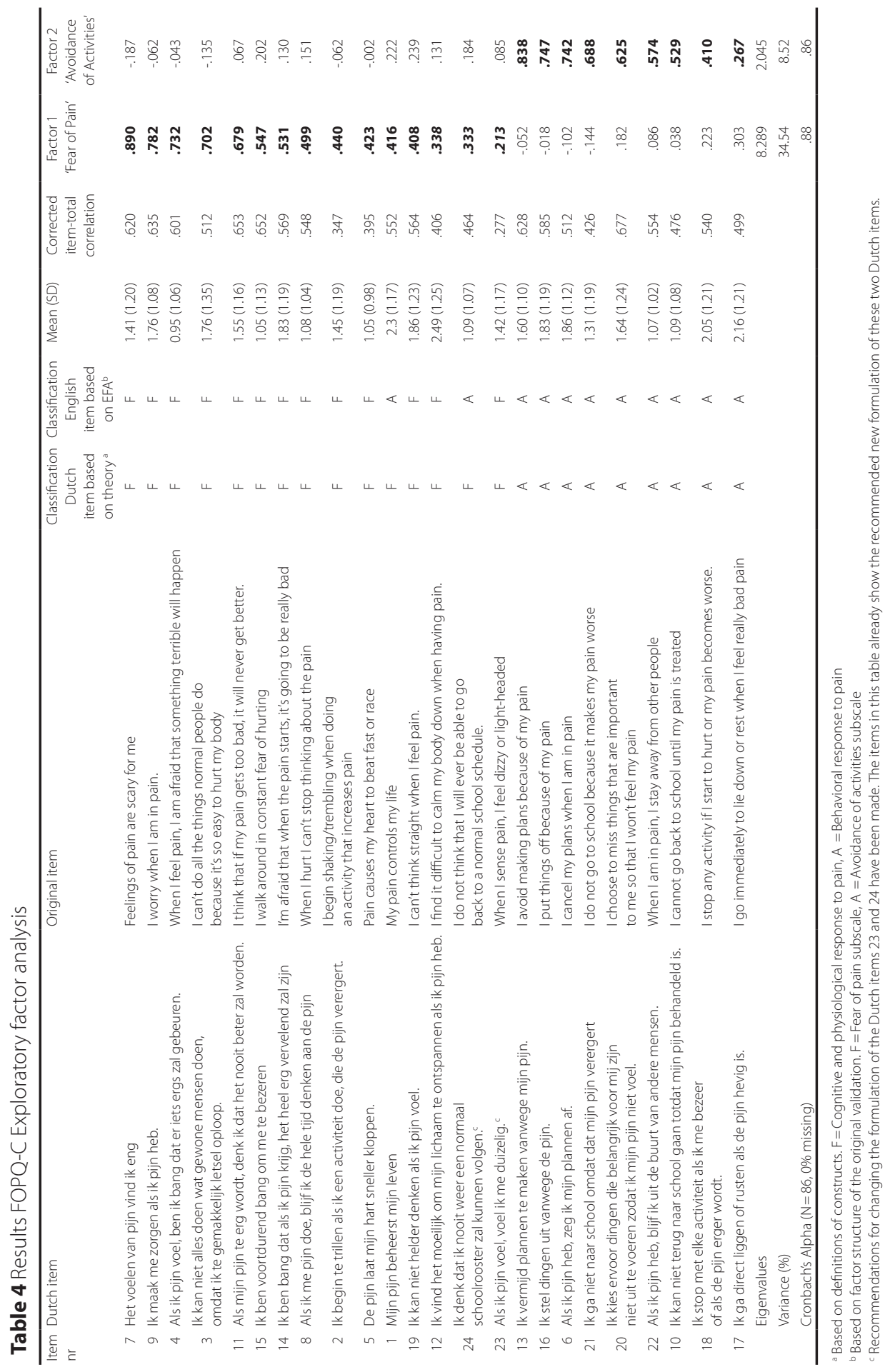


Table 5 Intercorrelations FOPQ-C, PCS, FDI and pain intensity for hypothesis testing

\begin{tabular}{|c|c|c|c|c|c|c|c|c|c|}
\hline Variable & 1 & 2 & 3 & 4 & 5 & 6 & 7 & 8 & 9 \\
\hline 1 FOPQ-C Total & - & $.94^{\mathrm{b}}$ & $.86^{b}$ & $.76^{\mathrm{b}, \mathrm{H}}$ & $.70^{\mathrm{b}}$ & $.71^{\mathrm{b}}$ & $.66^{\mathrm{b}}$ & $.58^{\mathrm{b}, \mathrm{H}}$ & $.14^{\mathrm{H}}$ \\
\hline 2 FOPQ-C Fear & & - & $.64^{\mathrm{b}, \mathrm{H}}$ & $.78^{\mathrm{b}, \mathrm{H}}$ & $.72^{\mathrm{b}}$ & $.73^{b}$ & $.69^{b}$ & $.54^{\mathrm{b}}$ & .10 \\
\hline 3 FOPQ-C Avoidance & & & - & $.56^{\mathrm{b}, \mathrm{H}}$ & $.52^{\mathrm{b}}$ & $.52^{\mathrm{b}}$ & $.48^{b}$ & $.52^{\mathrm{b}}$ & .17 \\
\hline 4 PCS-C Total & & & & - & $.92^{\mathrm{b}}$ & $.78^{\mathrm{b}}$ & $.94^{\mathrm{b}}$ & $.42^{\mathrm{b}}$ & .15 \\
\hline 5 PCS-C Rumination & & & & & - & $.68^{\mathrm{b}}$ & $.77^{b}$ & $.35^{\mathrm{b}}$ & .07 \\
\hline 6 PCS-C Magnification & & & & & & - & $.61^{\mathrm{b}}$ & $.22^{\mathrm{a}}$ & -.02 \\
\hline 7 PCS-C Helplessness & & & & & & & - & $.46^{\mathrm{b}}$ & $.25^{\mathrm{a}}$ \\
\hline $8 \mathrm{FDI}$ & & & & & & & & - & $.45^{\mathrm{b}}$ \\
\hline 9 Pain intensity & & & & & & & & & - \\
\hline
\end{tabular}

\section{Reformulations in the Dutch version of the FOPQ}

For item 23 ('When I sense pain I feel dizzy or light-headed') and 24 ('I do not think I will ever be able to go back to a normal school schedule') it is recommended to change the formulations in the Dutch version. Item 23 contains a 'double' formulation, which might result in ambiguous answers. It is recommended to change the item into 'When I sense pain, I feel dizzy'. Item 24 is deemed very difficult to answer for adolescents, since this item contains a double negative, which is required to be answered by either agreeing or disagreeing. It is recommended to change the item to 'I think I can never go back to a normal school schedule'. In table 4, the new formulations of the Dutch items are already incorporated.

\section{DISCUSSION}

The aim of this study was to investigate internal consistency and construct validity of a systematically translated version of the FOPQ-C. After translation of the FOPQ-C items into Dutch, exploratory factor analysis resulted in a two factor structure. The total scale (24 items), 'Fear of Pain' subscale (15 items) and 'Avoidance of Activities' subscale (9 items) of the FOPQ-C all had high Cronbach's a (> .86), meaning internal consistency is good (14). Furthermore, hypotheses testing for construct validity resulted in 5 out of 6 confirmed hypotheses, and is therefore evaluated as good (14).From the perspective of clinical relevance it is important to take the formulated conceptual framework into consideration. The relationships between the outcomes of the FOPQ-C and the outcomes of the constructs regarding pain catastrophizing and pain intensity were as expected and in line with the literature; a moderate to high correlation between pain related fear and pain catastrophizing (5) and a very low positive relation with pain intensity $(4,5)$. However, the relationship between the outcomes regarding pain related fear and the outcomes of func- 
tional disability seemed a little bit stronger in a positive direction as expected in this population. Although higher than hypothesized, similar strengths of correlations were found in a correlation between functional disability (FDI) with the PASS-20 that measures fear and anxiety responses specific to pain, and for the relationship between the FDI and the Multidimensional Anxiety Scale for Children (MASC) that measures symptoms of anxiety in children and adolescents (18).

For clinical practice the results of this study imply that the Dutch version of the FOPQ-C can be used in practice to assess fear of pain and avoidance in adolescents presenting with musculoskeletal chronic pain complaints. When using a biopsychosocial perspective and the (interpersonal) fear avoidance model as a foundation for adolescent (outpatient) chronic pain rehabilitation treatment, the identification of fear of pain is an essential step. The presence (or absence) of fear of pain and avoidance adds to the image of the patient that a therapist can obtain during pre-treatment screening and reveals possible treatment targets. Treatments, like Graded Exposure in vivo, aim to increase functional ability by targeting fear of pain or (re)injury and catastrophic thinking. Until now, no measure was available for Dutch (and Flemish) speaking patients that enabled the assessment of fear of pain and avoidance.

When comparing the groups of participants in the original validation and the validation of the Dutch translation, the samples are comparable but there are some differences to be mentioned. Compared with the USA sample, the Dutch sample was approximately two years older on average, and an $8 \%$ higher percentage of females were present. Pain duration in the USA sample was 14 months on average. In the Dutch sample, the majority of the adolescents reported the onset of their current pain complaints between 1 and 5 years ago. On average, the pain diagnoses were similar, although the Dutch sample seems to be slightly more homogeneous. Furthermore, the Dutch sample scored 4 points lower on the mean FOPQ-C total score and scored lower on the PCS-C total score. The level of disability (FDI) was the same in both samples, classifying these patients as moderately disabled (20). Last, pain intensity/rating was comparable.

Furthermore, the exploratory factor analysis of the Dutch version of the FOPQ-C resulted in a similar 24-item, two-factor structure as the original (USA) version, allowing the same factor names 'Fear of Pain' and 'Avoidance of Activities'. Some differences compared to the original version exist. First, the original Fear of Pain subscale contained 13 items, but the Dutch version resulted in 15 items in the Fear of Pain subscale. Consequently, the Dutch version contained 9 items in the Avoidance of Activities subscale in contrast to 11 items in the original version. Item 1 'Pain controls my life' and item 24'I do not think that I will ever be able to go back to a normal school schedule' loaded on the Avoidance subscale in the original version, but load on the Fear of Pain subscale in the Dutch version. Since the interpretation of the translated items was not tested in the target population, an explanation why these items changed subscales cannot be provided.

Moreover, item 12, 17, 23 and 24 were kept in the analysis despite a loading <.4, but on grounds of clinical relevance. The rather small sample size could be a certain influence on the factor loadings. The factor loadings of items 12, 23 and 24 are in favour of the factor that describes the cognitive and physiological response to pain related fear, which is in line with the theoretical concept of this factor. The three items express a physiological or cognitive response to pain related fear. Although item 17 had similar factor loadings on both factors, this item was placed within 
the 'Avoidance of Activities' subscale, based on the theoretical construct of the subscale. Based on the results of the analysis, recommendations for changing the formulation of item 23 and 24 have been made by the researchers.

In this study, some assumptions have been made. Additionally, some limitations deserve to be mentioned. First, after translation, the FOPQ- $C$ was approached as a 'new' measurement instrument and exploratory factor analysis was chosen as a means to determine factor structure. Confirmatory factor analysis was not performed because of the limited sample size (13). Although data of only 86 participants was available for the validation, the high Kaiser-Meyer-Olkin measure indicated the correlation matrix derived from the data was appropriate for factor analysis (21). Second, the data used in the analyses was moderately skewed, yet, parametric testing (Pearson correlations) was continued, assuming normality of the sampling distributions. Third, it was assumed that a continuous variable underlay the Likert-scale answering scale, so that it was possible to proceed with factor analysis methods for continuous data (12).

In this initial validation, focus was on factor structure, internal consistency and construct validity of the Dutch version of the FOPQ-C. This is only a part of the measurement properties that are important in assessing methodological quality of measurement instruments (22). To increase the FOPQ-C's usefulness in clinical practice and research it is worthwhile to investigate more properties, for example the content validity and responsiveness to change of the FOPQ- $C$. It would be worthwhile to investigate the understandability and the way in which the individual items of the FOPQ-C are interpreted by the target population. Analysis of the current data have shown that there might be issues with the interpretation of the items. This step was not performed in this study. Responsiveness to change is of interest, especially if the measure is intended to be used for evaluating changes in levels of fear of pain and avoidance as a result of treatment or in research (23). An important prerequisite for investigating responsiveness is to evaluate reliability (test-retest-reliability), which was not possible with the data for this validation. Further validation studies might focus on these aspects.

\section{Conclusion}

This Dutch version of the Fear of Pain Questionnaire demonstrated good internal consistency and good construct validity in a sample of adolescents (11-22 years) seeking help for chronic musculoskeletal pain. The relationship between the outcomes of the FOPQ-C and the outcomes of the FDI are more positive than expected on a theoretical basis. The Dutch version of the FOPQ-C can be used in Dutch rehabilitation care to identify the presence of pain related fear in the context of musculoskeletal chronic pain complaints. 


\section{ACKNOWLEDGEMENTS}

We would like to thank all participants in this study for filling in all questionnaires. Furthermore, we thank Diane Crook (DC) and Liesbeth Goubert (LG) for their help in the translation process. CD was able to work on the validation of the FOPQ-C because of a grant of Fonds Nuts Ohra.

\section{DECLARATION OF INTEREST}

Funding None declared

Conflicts of interest None declared 


\section{REFERENCES}

1. King S, Chambers CT, Huguet A, MacNevin RC, McGrath PJ, Parker L, et al. The epidemiology of chronic pain in children and adolescents revisited: A systematic review. Pain. 2011;152(12):2729-38.

2. Eccleston C, Jordan AL, Crombez G. The impact of chronic pain on adolescents: a review of previously used measures. J Pediatr Psychol. 2006;31(7):684-97.

3. Vlaeyen JW, Linton SJ. Fear-avoidance and its consequences in chronic musculoskeletal pain: a state of the art. Pain. 2000;85(3):317-32.

4. Simons LE, Kaczynski KJ. The Fear Avoidance model of chronic pain: examination for pediatric application. The Journal of Pain 2012;13(9):827-35.

5. Simons LE, Sieberg CB, Carpino E, Logan D, Berde C. The Fear of Pain Questionnaire (FOPQ): Assessment of Pain-Related Fear Among Children and Adolescents With Chronic Pain. The Journal of Pain. 2011;12(6):677-86.

6. Goubert L, Simons LE. Cognitive styles and processes in paediatric pain. In: McGrath PA, Stevens BJ, Walker SM, Zempsky WT, editors. Oxford Textbook of Paediatric Pain. Oxford: Oxford University Press; 2014. p. 95-101.

7. Crombez G, Bijttebier P, Eccleston C, Mascagni T, Mertens G, Goubert L, et al. The child version of the pain catastrophizing scale (PCS-C): a preliminary validation. Pain. 2003;104(3):639-46.

8. Walker LS, Greene JW. The functional disability inventory: measuring a neglected dimension of child health status. J Pediatr Psychol. 1991;16(1):39-58.

9. Claar RL, Walker LS. Functional assessment of pediatric pain patients: psychometric properties of the functional disability inventory. Pain. 2006;121(1-2):77-84.

10. Flowers SR, Kashikar-Zuck S. Measures of juvenile fibromyalgia: Functional Disability Inventory (FDI), Modified Fibromyalgia Impact Questionnaire-Child Version (MFIQ-C), and Pediatric Quality of Life Inventory (PedsQL) 3.0 Rheumatology Module Pain and Hurt Scale. Arthritis Care Res (Hoboken). 2011;63 Suppl 11:S431-7.

11. Stinson JN, Kavanagh T, Yamada J, Gill N, Stevens B. Systematic review of the psychometric properties, interpretability and feasibility of self-report pain intensity measures for use in clinical trials in children and adolescents. Pain. 2006;125(1-2):143-57.

12. Gaskin CJ, Happell B. On exploratory factor analysis: a review of recent evidence, an assessment of current practice, and recommendations for future use. Int J Nurs Stud. 2014;51(3):511-21.

13. De Vet HC, Terwee CB, Mokkink LB, Knol DL. Measurement in Medicine. Cambridge: Cambridge University Press; 2011.

14. Terwee CB, Bot SD, de Boer MR, van der Windt DA, Knol DL, Dekker J, et al. Quality criteria were proposed for measurement properties of health status questionnaires. J Clin Epidemiol. 2007;60(1):34-42.

15. Page MG, Fuss S, Martin AL, Escobar EM, Katz J. Development and preliminary validation of the Child Pain Anxiety Symptoms Scale in a community sample. J Pediatr Psychol. 2010;35(10):1071-82.

16. Huguet A, McGrath PJ, Pardos J. Development and preliminary testing of a scale to assess pain-related fear in children and adolescents. The Journal of Pain 2011;12(8):840-8.

17. Leeuw M, Goossens ME, Linton SJ, Crombez G, Boersma K, Vlaeyen JW. The fear-avoidance model of musculoskeletal pain: current state of scientific evidence. J Behav Med. 2007;30(1):77-94. 
18. Martin AL, McGrath PA, Brown SC, Katz J. Anxiety sensitivity, fear of pain and pain-related disability in children and adolescents with chronic pain. Pain research \& management. 2007;12(4):267-72.

19. Caes L, Fisher E, Clinch J, Tobias JH, Eccleston C. The role of pain-related anxiety in adolescents' disability and social impairment: ALSPAC data. Eur J Pain. 2015;19(6):842-51.

20. Kashikar-Zuck S, Flowers SR, Claar RL, Guite JW, Logan DE, Lynch-Jordan AM, et al. Clinical utility and validity of the Functional Disability Inventory among a multicenter sample of youth with chronic pain. Pain. 2011;152(7):1600-7.

21. Dziuban CD. When is a correlation matrix appropriate for factor analysis? Some decision rules. Psychol Bull. 1974;81(6):358.

22. Mokkink LB, Terwee CB, Patrick DL, Alonso J, Stratford PW, Knol DL, et al. The COSMIN study reached international consensus on taxonomy, terminology, and definitions of measurement properties for health-related patient-reported outcomes. J Clin Epidemiol. 2010;63(7):737-45.

23. Kimberlin CL, Winterstein AG. Validity and reliability of measurement instruments used in research. Am J Health Syst Pharm. 2008;65(23):2276-84. 



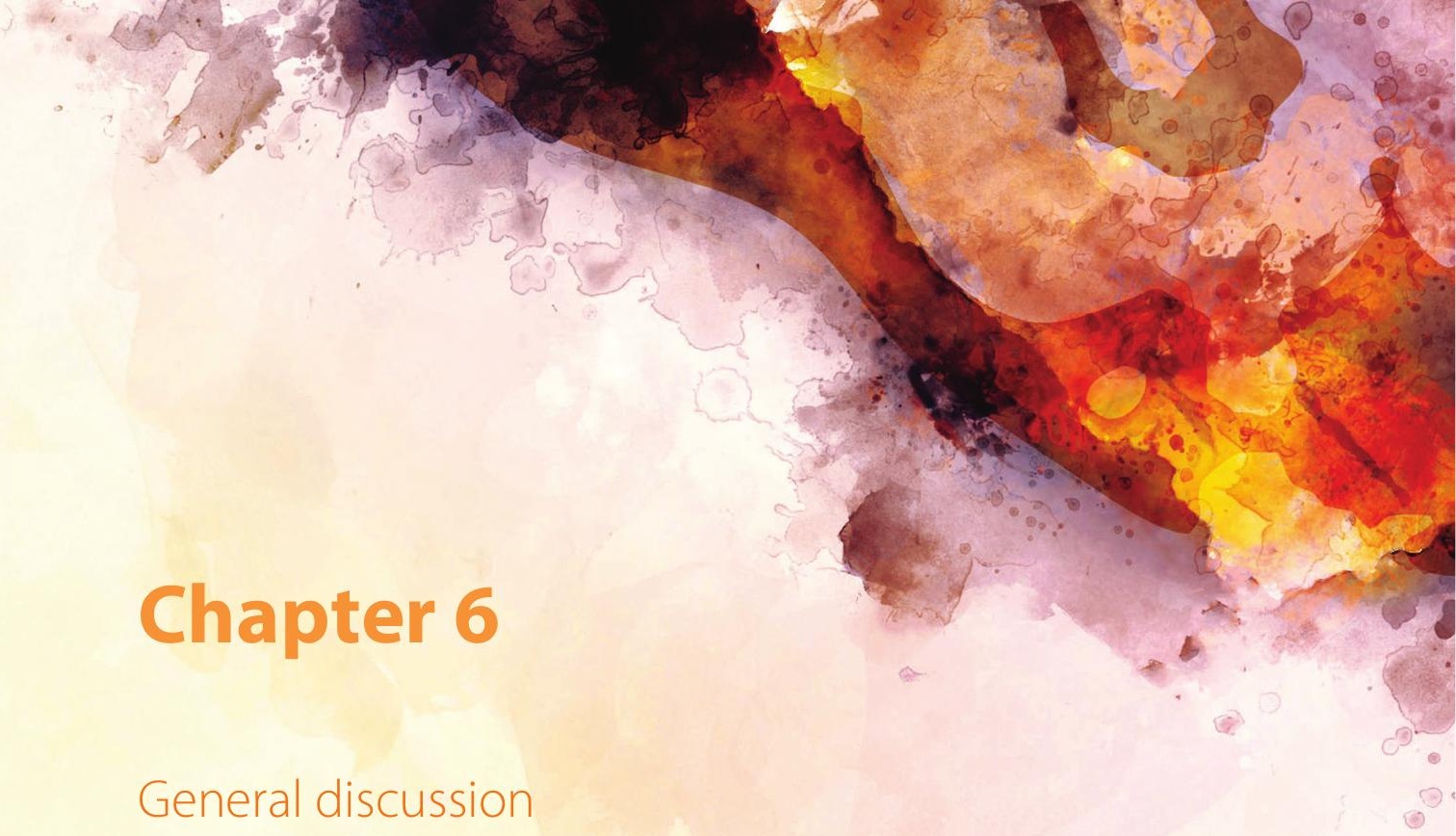





\section{GENERAL DISCUSSION}

The main aim of this dissertation was to add to the scientific evidence on rehabilitation treatment for adolescents with chronic musculoskeletal pain, with the ultimate goals to ameliorate negative consequences of the adolescent's complaints and to increase their quality of life. The efforts towards reaching this goal consisted of the performance of a multicentre randomized clinical trial and a validation study of a measurement instrument. In the randomized clinical trial, the effectiveness in decreasing functional disability of an interdisciplinary graded exposure program targeting pain-related fear for adolescents with chronic musculoskeletal pain was compared to usual care. Additionally, the feasibility of the new exposure program was investigated in terms of protocol adherence by the treatment teams offering this program. The validation study concerned the translation of the Fear of Pain Questionnaire - Adolescent version into Dutch, and an evaluation of the internal consistency and construct validity of this questionnaire.

In this chapter, the main findings of these studies are presented and discussed. Thereafter, an overview of methodological considerations, the implications for practice and recommendations for further research will be provided. In the end, a conclusion is drawn.

\section{Main findings}

What is the effectiveness of the interdisciplinary graded exposure program in decreasing functional disability in adolescents with chronic musculoskeletal pain?

Adolescents with chronic musculoskeletal pain, treated with the new interdisciplinary graded exposure program, showed a clinically relevant and statistically significant improvement on functional disability compared to usual care at 2, 4, 10 and 12 months after the start of treatment. In the exposure program, adolescents had an average improvement from moderate disability to light or no disability (FDI scores between 0-12) (1). On average, adolescents receiving usual care remained in the moderately disabled category (FDI scores between 13-29) (1). The results show that the magnitude of the estimated difference between 10 and 12 months decreased slightly. This decrease is difficult to interpret, since at those time points only 22 adolescents still completed the questionnaires. Missing questionnaires at these time points could not be related to any measured variables, which makes selective drop out of the study unlikely. Moreover, the magnitude of the decrease still remains well within a clinically relevant change of eight FDI points (2). Therefore, this decrease is not considered to be of significant importance. Furthermore, perceived harmfulness of previously avoided activities and social situations also decreased significantly more in the exposure program group compared to usual care, at all time points. For the remainder of the secondary outcome measures, results differed per time point. No significant differences were found for fear of pain at all time points. Pain catastrophizing differed only at 2 months after the start of the intervention. Depressive symptoms showed a difference at 10 months after the start of treatment, and pain intensity showed differences at 4 and 10 months after start of treatment. For health related quality of life, differences in improvement in favour of the exposure program was 
visible at 2 and 10 months for the domain of psychological functioning and at 2 and 4 months for the domain of functional status. No differences were visible for the domains of physical status and social functioning (Chapter 3).

To our knowledge, this was the first RCT evaluating the effectiveness of an interdisciplinary exposure program targeting pain-related fear in order to decrease functional disability in adolescent with chronic musculoskeletal pain. Comparison to other studies investigating an exposure intervention is therefore not possible. When relating the results of our RCT to other interdisciplinary pain programs, Hechler and colleagues (3) published a randomized controlled trial comparing an interdisciplinary pain program with changes within a waiting-list control group. This RCT demonstrated that a 3-week intensive inpatient interdisciplinary pain program significantly improved pain-intensity, disability and school absence in a combined endpoint evaluation as compared with the waiting-list control condition. Although both RCTs report positive results in reduction of disability, there are several differences that need to be described. First, the study sample in the RCT of Hechler and colleagues (3) consisted mainly of patients with chronic headache (approximately $75 \%$ ) and was slightly younger (9-17 years, mean age was 14 years) than our patients with chronic musculoskeletal pain aged 12-21 years old (mean age 16 years). Furthermore, in Hechlers RCT adolescents were classified as severely disabled, with a mean score of 39 (SD=9) on the Paediatric Pain Disability Index, whereas the adolescents with chronic musculoskeletal pain in our RCT were on average moderately disabled at baseline. Second, the interventions differed in duration of the treatment and treatment setting. Hechler evaluated a 3-week 5-8 hours a day inpatient treatment. Our program being a 7 week 2 hours a week (or 15 weeks in case of physical training) outpatient treatment. Compared to Hechler, adolescents in our RCT received less treatment hours, but a decrease in functional disability was achieved. Third, the RCTs differed in the choice of comparator intervention. With a treatment duration of 3 weeks Hechler and colleagues (3) opted for a waiting-list control group, whereas the best available, most used treatment (care as usual) was chosen as parallel group comparison in our RCT. The choice of comparator intervention is related to the different goals of the studies, Hechler opting for evaluation of treatment efficacy as opposed to our aim of studying treatment effectiveness. Consequently, the results of our RCT are more applicable to daily rehabilitation care. In adults, evidence has been obtained supporting that patients can experience significant deterioration in health related quality of life and psychological well-being while waiting for treatment for chronic pain for 6 months. Even with waiting times of 10 weeks or less deterioration has been reported (4). Therefore, comparing the new treatment to usual care was considered more tolerable than comparing to a waiting-list control group. Furthermore, comparing two different active interventions in our RCT, allowed for evaluation of group differences in a long term follow up. Fourth, comparisons with regard to changes in fear are difficult to make. Although Hechler and colleagues report significant reductions in pain catastrophizing and general anxiety in the intervention group at all time points, different measurement instruments have been used and a measure of pain-related fear was not used. Fifth, the interventions in both RCTs actively involved parents in the treatment. Although both treatment programs aimed to change parental cognitions and behaviours, results have not been reported. The program for parents that was part of our exposure program has earlier been evaluated for its feasibility and also evaluated changes in parental behavioural factors pre- and 
post-treatment (5). Results showed that parents rated the parent module as highly acceptable. Additionally, significant improvements in self-reported catastrophizing about their adolescent's pain, fear of pain and perceived functional disability of their adolescent were observed between pre- and post-treatment. Furthermore, since parents participated in the exposure program in our $\mathrm{RCT}$, their influence on the outcomes for the adolescents is part of the results on adolescent outcomes that have been reported.

How feasible was the exposure program in a specialized outpatient rehabilitation setting?

Evaluation of audio- and video recordings of program sessions, and registration of adolescent and parent attendance, demonstrated that the new exposure program was largely performed according to protocol, with high treatment fidelity. Adolescents reported the program to be patient-centred and parents reported the program to be family-centred. Moreover, treatment teams of the participating rehabilitation centres had a favourable opinion about the program and intended to implement the new program in their treatment centre. These findings led to the conclusion that the program was feasible to work with and implementation was recommended in case of demonstrated treatment effectiveness.

For evaluation of treatment fidelity, an adapted Method of Assessing Treatment Delivery (MATD) was used. Adequate interrater reliability and content validity (evaluated as the agreement between two experts of the treatment protocols in allocating treatment elements to previously defined categories) were established for the MATD when applied for this purpose (6). Within the context of the process evaluation, only protocol adherence of the treatment team to the exposure program protocol was evaluated. In the RCT, analysis of treatment fidelity of the exposure program was expanded by incorporating assessment of treatment contamination by the alternative treatment to the evaluation. With approximately equal amounts of treatment contamination (5\% in the exposure program and $8 \%$ in care as usual), and high protocol adherence (81\%) in the exposure program, these findings contribute to our confidence that an honest comparison between the two interventions has been made.

In this matter, a critical note is in place, as the MATD can only be used to assess some but not all components of treatment fidelity. The limitation of this method will be described in the paragraph on methodological considerations in this discussion section.

\section{Assessment of fear of pain in Dutch adolescents with chronic musculoskeletal pain}

To enable the use of the Fear of Pain Questionnaire Child version (FOPQ-C) in Dutch adolescents with chronic musculoskeletal pain, the original questionnaire was translated into Dutch, and psychometric properties were evaluated. In the Dutch version, exploratory factor analysis confirmed the original two-factor structure of the measure, with both factors demonstrating good internal consistency. Hypothesis testing for construct validity resulted in five confirmed hypotheses out of six formulated hypotheses. Therefore, construct validity was concluded to be good as well. Hypotheses were formulated based on prior research and theory about the direction and strength 
of the correlations between the Dutch FOPQ-C and the different comparator variables (Pain Catastrophizing, Functional Disability and pain intensity). Furthermore, it was recommended to change the formulation of two items in the questionnaire, to simplify comprehension of these items. For clinical practice, the results of this study imply that the Dutch version of the FOPQ-C can be used in practice, to assess fear of pain, and avoidance of activities, in adolescents presenting with chronic musculoskeletal pain. Identification of the presence (or absence) of fear of pain and avoidance in adolescents is an important step in pre-treatment screening, as it can reveal possible treatment targets.

The assessment of fear of pain in Dutch adolescents could be expanded by translating and validating additional fear of pain questionnaires in the same line, and in doing so, providing a full assessment of the presence of fear of pain in the adolescent within the family context. The interpersonal fear avoidance model describes the interaction between adolescent and parent cognitive-affective and behavioural factors, including pain-related fear. Within this model, parents interpret their adolescents pain behaviour through the lens of their own pain-related fears and behave accordingly $(7,8)$. Therefore, an additional measure was developed that assesses parent pain-related fears in the context of their adolescent's pain, the Parent Fear of Pain Questionnaire (PFOPQ), demonstrating sound psychometric results (9). A cross-cultural validation of the PFOPQ would be a useful addition to the Dutch version of the Fear of Pain Questionnaire Child version, enabling a comprehensive assessment of fear of pain in the interpersonal context of fear of pain in adolescents. Furthermore, the Fear of Pain Questionnaire - parent report (FOPQ-P) exists, that can be used as a parent proxy report to assess pain-related fear in children and adolescents with chronic pain. The FOPQ-P has also been systematically translated into Dutch and data have been collected to analyse its psychometric properties. Analysis of this data is planned.

\section{Can we put a price on adolescent pain?}

Although the health consequences and burden on the families are significant, and the prevalence rates of adolescent chronic pain are high, there are currently no solid estimates of the economic burden of adolescent chronic pain (10). However, this information can be very helpful for policy makers to allocate resources for adolescent chronic pain treatment and research (10). Furthermore, with regard to potential cost savings by treating or preventing chronic pain, little is known. Only a few studies have been performed. In 2014, Hechler and colleagues (3) presented their results on the economic effects of the RCT on the 3-week intensive interdisciplinary pain treatment for children with severe chronic pain. For adolescents who improved after treatment, adolescent health service utilization, parental work absenteeism, parental work days lost and parental subjective financial burden decreased significantly in both short (6 months) and long (12 months) term. For adolescents who did not improve after treatment, adolescent health service utilization, parental work absenteeism and parental work days lost only improved in the long term. More recently, in 2018, Law and colleagues (11) showed that in children and adolescents with chronic pain, health care expenditures decreased significantly from the year prior to adjunctive internet cognitive behavioural therapy or adjunctive internet education to the year following these interventions. Further, no differences in the rate of change in health care cost between both 
interventions were found in the RCT by Law and colleagues (11). Additionally, Evans, Benore and Banez (12) estimate a saving of $\$ 27,119$ per family in the year following admission to a 3-week intensive multidisciplinary chronic pain rehabilitation treatment. Savings were mainly in reduced health care utilization and reductions in parental missed work.

Nowadays, given that health care resources become increasingly scarce, demonstration of cost-effectiveness of new treatments becomes increasingly important in the decision to adopt new treatments in daily care. A future challenge is to analyse the available cost-effectiveness data of both interventions.

\section{Methodological considerations}

Although it has been reported that the methodological quality of RCTs in children and adolescents with chronic pain has improved over the years (13), researchers are still challenged when it comes to performing studies in this field. Some of these challenges had to be dealt with in our studies and will be described below.

The power is in the numbers: sample size and recruitment

One of the major problems in RCTs in children and adolescents with chronic pain is the relatively small sample size in each trial. One meta-analytic review, including 25 trials with 1247 young people with headache, abdominal pain and fibromyalgia, reported that the average trial arm size was 21 subjects (13). The smaller and more heterogeneous the sample size of a trial, the larger an effect needs to be in order to be found with adequate statistical power. This increases the risk of small treatment effects to be overlooked in case a priori calculated sample sizes for RCTs are not achieved. Many researchers struggle with the sample size because of a relatively small number of patients with a particular chronic pain problem at a single study site (14). Therefore, a recommended solution is the design of multicentre studies to increases sample sizes and accompanying statistical power (13).

In our RCT, to increase the chance of attaining the a priori calculated sample size of 124 adolescents, four treatment centres were recruited to participate in the RCT. The length of the recruitment period was determined, based on the number of adolescents treated in the year before the RCT started in each of these centres. The sample size calculation used (unpublished) data on the primary outcome measure functional disability (Functional Disability Inventory) of a clinical population of adolescents that were treated in one of the participating centres before the RCT started. The expected mean difference in FDI score between the intervention and control condition was set at 5 FDI points, which equalled approximately a 25\% difference in mean FDI score between the both randomized groups.

Even after a prolonged recruitment period of 25 months, in total only 60 adolescents were enrolled in the RCT. Increased efforts to enhance recruitment did not lead to the desired number of participants. These efforts consisted of activities such as prolonging the inclusion period, and increasing awareness of the treatment possibilities for adolescent chronic musculoskeletal pain amongst referring physicians, increasing treatment capacity, raising awareness for treatment 
possibilities for adolescent chronic pain in the patient's association and publishing about the treatment possibilities in local (medical) monthly magazines. Factors that contributed to the lower recruitment are the fact that adolescents simply declined participation to a scientific study (almost 1/3 of the invited participants declined for various reasons) that involved some extra efforts as compared to normal treatment outside the RCT. Further, identification of pain-related fear was found to be challenging by the newly trained treatment teams.

\section{A pragmatic approach to the RCT}

The RCT was designed to determine the effect of the new interdisciplinary graded exposure program on functional disability, and efforts were made to study this new intervention in the setting in which the program ultimately was intended to be offered in case of positive trial outcomes. These intentions have led to design choices made to maximize the applicability of the results to usual care settings, albeit warranting the internal validity of the trial (15). Based on the domains used in the PRECIS-2 tool (15), design choices will be discussed that either constitute to higher applicability of the trial results or lower applicability (less pragmatic, more explanatory). Choices that were made that enhance the applicability of the RCT results were made in the domains of eligibility, recruitment, setting, flexibility in the adherence of adolescents and parents to the program, and the choice of the primary outcome and analysis.

First, eligibility criteria for adolescents were equal to eligibility criteria for the investigated experimental intervention (exposure program) outside a research setting, which allowed for enrolment of a heterogeneous population of adolescents with musculoskeletal chronic pain comparable with the real-life situation. The formulation of the eligibility criteria aimed to incorporate all adolescents who would be referred to the new program if it was offered in usual rehabilitation care. Since the focus of the RCT was on adolescents with chronic musculoskeletal pain, the age range was confined to youth aged 12-21 years old. Second, with regard to the recruitment of the adolescents, a more pragmatic strategy was followed as well. Patients who were already referred to rehabilitation care presenting with chronic musculoskeletal pain were invited to participate. As the rate of recruitment was slower than anticipated, the efforts made to increase adolescent enrolment involved contacting referring physicians. However, at all times, awareness of treatment possibilities for our target population in rehabilitation setting was raised. Referring physicians were not encouraged to specifically refer potential participants to the study. Third, the setting in which the trial was executed is the same as the setting in which the results are likely to be implemented (15). The four participating centres were all centres offering rehabilitation care for children and adolescents with chronic musculoskeletal pain. Two of the four centres were paediatric rehabilitation centres, offering both outpatient and inpatient rehabilitation care. These centres typically provide rehabilitation care for children up to 18 years old. The other two centres were department of rehabilitation medicine of both an academic and a general hospital, offering outpatient rehabilitation care only. These centres also included adolescents up to 21 years old. 
This diversity of treatment centres also reflects the diversity of settings in which implementation of the new exposure program is intended. Fourth, flexibility of adherence by the adolescents and parents was high, in the sense that only treatment teams encouraged them to engage in the programs as best they could, equally as they would have done outside the study. Last, the primary outcome chosen, functional disability, is one, relevant to participants within a rehabilitation treatment, as they suffer from disability when entering treatment and treatment is aimed at decreasing the disability. In order to evaluate this decrease, intention to treat analysis was performed.

With respect to the organisation of care delivery, the follow up of adolescents and parents, and the flexibility of intervention delivery (especially of the exposure program), the design choices lean more towards the explanatory end of the explanatory-pragmatic continuum (15). Due to the delivery of the interventions in the context of an RCT, some essential study procedures interfered with the normal delivery of the treatment programs to the adolescents in usual care. For example, because randomization of the adolescents to one of the interventions was executed after informed consent was obtained, adolescents were informed at a later point in time what treatment program they were about to be offered. Simultaneously, consultants in rehabilitation medicine could only provide very succinct information about either intervention to the adolescents and parents, because randomization was performed after this visit. Therefore, also consultants in rehabilitation medicine were informed about treatment allocation at a later point in time. Furthermore, adolescents and their parents were asked to complete measurements on 3 extra occasions on top of the two regular (before and after treatment) screening and evaluation measurements. The extra measurements were necessary to obtain data on the long term follow up after the interventions.

Additionally, little flexibility in treatment delivery of the new exposure program was allowed in the RCT, something that is more closely related to an explanatory than a pragmatic approach to the study (15). This had the advantage that it allowed for evaluation of protocol adherence by the treatment teams offering the exposure program, because they all used the same protocol. Moreover, three of the four centres were newly trained in delivery of the exposure program, therefore delivery of the exposure program was not part of their routine yet. Working according to the exposure protocol could have given these teams some hold. For the new exposure program, all centres were encouraged to implement the program protocol exactly as planned, in terms of scheduling the program sessions, duration of the sessions and content of the sessions delivered to the adolescent and parents. Program protocols were distributed to the treatment teams, that entailed a detailed description of each program session, the people and materials that were expected to be present, and an agenda for that particular treatment session. Treatment teams were encouraged to deliver the complete programs as planned, even, for example, when in a particular adolescent, treatment goals had already been achieved before the end of the program. In this situation, treatment teams focussed extra on generalization of the treatment gains in the daily life of the adolescent. Furthermore, treatment teams were asked to audio-and video record the program sessions they were offering, and were aware that these recordings would be used to 
assess their adherence to the program protocol. Hence, therapist performance may have been influenced because they were aware that during the intervention period, their performance would be recorded for analysis of protocol adherence.

Opposite to the low flexibility in the new exposure program, the care as usual intervention was affected as little as possible. Each treatment centre was allowed to continue usual care as it was described in their own treatment protocols. These were based on a consensus document of the Dutch working group for youth with chronic pain and fatigue. Certainly, essential study procedures had to be executed, however, differences between treatment centres in terms of treatment duration, planning and content were left untouched as far as possible. Only the contrast between the two investigated treatments was warranted by urging treatment teams not to contaminate the alternative intervention with essential treatment elements of one of the interventions. Last but not least, by employing randomization by minimization, blinding of the study team, treatment teams and adolescents/parents at the moment of treatment allocation, and concealed treatment allocation performed by an organization independent of the study, the risks of bias were minimalized (16).

To summarize, in six out of nine domains indicated by the PRECIS-2 tool (15), design choices were made that enhance applicability, the ability for the trial results to be used in rehabilitation setting. This enhances our confidence that the results can be generalized to daily rehabilitation practice, although more strict requirements on the delivery of treatment and analysis warrant the internal validity of the RCT and its results. Clinicians can therefore use the results of the RCT to decide whether they want to expand the treatment possibilities they have on offer for adolescent with chronic musculoskeletal pain reporting pain-related fear with a program specifically targeting pain-related fear to decrease functional disability.

Comparing to care as usual graded activity based treatment, bringing some competition into the game

Most previous performed RCTs evaluating treatment effects of psychological treatment on pain intensity, emotional distress and disability used waiting-list control or treatment as usual as control conditions $(13,17)$. In the RCT the exposure program was compared to usual care, generally comprising treatment based on the principles of graded activity therapy. At the start of the study, multidisciplinary graded activity based treatments were the most common form of treatment that was offered in outpatient rehabilitation setting in the Netherlands. The use of an active control condition allows determination whether the new treatment outperforms the care as usual treatment in decreasing functional disability in adolescents with chronic musculoskeletal pain reporting pain-related fear (18). The choice of an active treatment comparator has, however, also been criticized in the sense that this makes it difficult to separate a treatment effect from a placebo effect, therefore lowering trial quality (13). This is an argument which is more of interest in efficacy trials, situated on the explanatory end of the pragmatic-explanatory continuum (19). These efficacy trials explore if and how the investigated treatment works. These trials are designed to minimize bias and confounders, to maximize intervention effects $(16,20)$. Assigning children 
and adolescents to placebo (or attention-control) treatments does not align with our goal of evaluating which treatment would be the treatment of choice in real life (16), and is considered unacceptable by many (14).

Off the main road: using minimization as allocation method

Although supported by CONSORT (Consolidated Standards of Reporting Trials), the use of minimization is controversial (21). Minimization aims to generate balanced treatment arms with respect to predefined adolescent factors (age and gender in our RCT), thereby simultaneously balancing the number of adolescents in each trial arm during the whole recruitment period (22, 23). Even with the calculated 124 adolescents as desired trial size, this RCT would be a study with a small sample size. Since minimization has been shown to outperform simple randomization and stratified randomization methods (which in small trials are at risk of empty cells) in achieving balanced groups (22), this allocation method was preferred.

By all means, the use of minimization also has disadvantages. For example, one of the major critiques is that assignment can be predicted (22). However, this is also true for other allocation methods such as stratification and therefore not an argument to omit this technique. One way to overcome the problem of predictability is to add a random component to the allocation algorithm (23), as was done in our RCT. By arranging a concealed allocation procedure, performed by an organization completely independent of our RCT the risks of bias were further minimized.

\section{Evaluation of treatment fidelity, a detailed and limited procedure}

Evaluation of treatment fidelity, the extent to which a treatment is delivered as intended (24), can shed light on the genuineness of the comparison that is being made between treatments. The use of the Method of Assessing Treatment Delivery (MATD) was proven to be a reliable and valid method to assess some, but not all components of treatment fidelity (6). The MATD focuses on the assessment of treatment delivery in terms of protocol adherence by the treatment teams, contamination of the treatment by the alternative treatment and treatment differentiation. Three additional components that have been reported that comprise treatment fidelity, being treatment competence, treatment receipt by the patient and enactment of the patient upon treatment, are not evaluated with this $\operatorname{MATD}(25,26)$. Some insights into the treatment competence of the treatment teams offering the exposure program has been provided by describing the way in which treatment teams have been trained before start of the RCT. The other two components, concerning treatment receipt and enactment by the adolescents have not been evaluated, as this would have increased the burden of participating in the study to a degree that was considered too high. 
The unmissable ingredient in a recipe for high quality evidence: high quality measurement Awareness of pain in children has increased over the past three decades (27). This has resulted in increased research efforts into, for example, working mechanisms of chronic pain, treatment possibilities, and measurement instruments. Today, the populations, measurement instruments used, and selected outcomes are heterogeneous in RCTs in children and adolescents with chronic pain $(13,28,29)$. Although these efforts are important developments for this field of research and care, the diversity currently existing hinders the pooling of data for meta-analyses of treatment effects. Research into measurement instruments and their psychometric properties in different populations of adolescents with chronic pain (for example populations speaking different languages) can lead to a broader evidence base on high quality measures. This could benefit future RCTs on treatment effectiveness.

Measurement instruments form a basis in diagnosis, prognosis and evaluation of treatments in care and research in adolescent chronic pain (30). In 2006, Eccleston, Jordan and Crombez (31) performed a review on measurements that have previously been used to measure the impact of chronic pain in adolescents. At that time, the authors reported they identified 43 separate measurement instruments, for which clinically relevant psychometric data were missing for many of those instruments. Furthermore, it was concluded that in some domains of chronic pain experience, no routine assessment existed (31). Thereafter, in 2008, recommendations were made with regard to core outcome domains and measures that could be used in clinical trials with youth with chronic/recurrent pain complaints (32). Assessment of outcomes in pain intensity, physical functioning, emotional functioning, role functioning, symptoms and adverse events, global judgment of satisfaction with treatment, sleep and economic factors were recommended. However, no measures could be recommended in the domains of symptoms and adverse events, global satisfaction with treatment and economic factors, due to insufficient development of instruments in these domains $(32,33)$. Subsequently, in 2009 Palermo (33) evaluated the status of assessment of chronic pain in five of these eight core outcome domains. It is concluded that progress has been made in measurement of relevant outcome domains in the assessment of chronic pain in children. While there are still opportunities in further validation of existing measures in the domains of pain intensity, physical functioning, role functioning, emotional functioning, and sleep, one or more measures have been developed and validated in these domains (33). Now that researchers and clinicians are encouraged to, and focus on the evaluation of treatment effects on non-pain outcomes, such as functioning or costs $(3,11,13,29)$, it becomes increasingly important that high quality measures are available in all of these domains.

Advances have been made in the past years. In 2009, Palermo already pointed out that there was a need for theory describing the mechanisms through which pain influences non-pain outcomes such as functioning (33). With the introduction of the interpersonal fear avoidance model for adolescent chronic musculoskeletal pain (7), a model has been provided that describes a number of pain-related constructs and provides a mechanism though which pain influences functioning. As these theoretical models can be used to develop theory-driven treatments (like the exposure treatment presented in this dissertation), they can also be used to select appropri- 
ate measurement domains. Parallel to theory development, efforts have been made to develop measurement instruments that can be used to assess these pain-related constructs (e.g. pain catastrophizing, pain-related fear, avoidance behaviours, depression). One of these constructs receiving growing attention, previously mentioned, is pain-related fear. Recently, Fisher and colleagues (34) conducted a systematic review and meta-analysis on the assessment of pain anxiety, pain catastrophizing and pain-related fear in children and adolescents with chronic pain. They identified three measures of pain-related fear, developed from 2011 onwards, the Fear of Pain Questionnaire Child version (FOPQ-C) (35), the Paediatric Pain Fear Questionnaire (PPFQ) (36) and the Photograph Series of Daily Activities for youth (PHODA-Youth) (37). The FOPQ-C and PPFQ are similar questionnaires, both containing items cognitive, behavioural and physiological responses about pain. Both questionnaires contain two subscales, representing a cognitive response to pain and a behavioural/physiological response. The PHODA-Youth is different, measuring those age-appropriate activities that are avoided by adolescents due to pain-related fear. For measuring pain anxiety, only one measure was identified, being the Bath Adolescent Pain Questionnaire (BAPQ) pain-specific anxiety subscale (38). For measuring pain catastrophizing, three measures were identified. The Pain Catastrophizing Scale for Children (PCS-C) (39) has been used most often. The two other measures were subscales of coping measures, being the Pain Response Inventory catastrophizing subscale (PRI)(40) primarily used in children with abdominal pain, and the Pain Coping Questionnaire internalizing/catastrophizing subscale (PCQ-IC) (41). All these measures but one (the PPFQ) were rated as well established by the authors in this review (34). The authors further conclude that the results demonstrated strong-moderate correlations with other fear-avoidance model constructs such as pain intensity, disability, general anxiety and depression, supporting that more validated measures have become available for a broad assessment of pain-related constructs in children and adolescents.

Another development, also visible in the area of measurement instruments, is the recognition of the importance of parental influences in adolescent chronic pain. This is reflected in the development of both adolescent and parent versions of different measurement instrument, such as the Fear of Pain Questionnaire child and parent version (35) including the Parent Fear of Pain Questionnaire (9) and the Pain Catastrophizing Scale child version (39) and parent version (42).

When selecting measurement instruments to assess the chosen domains, researchers and clinicians need to be aware that there are different purposes of measurement, diagnosis, evaluation of therapy and prediction of future course (30). With each different purpose, different psychometric properties of the measurement instrument are required. For example, for diagnostic purposes, such as identifying the presence of pain-related fear in adolescents with chronic musculoskeletal pain, the measurement instrument has to be able to discriminate between adolescents who are fearful and adolescents who are not. Researchers and clinicians could evaluate reliability, validity and interpretability of different measurement instruments when making a choice $(43,44)$. To evaluate the effects of a treatment, such as the effect of the exposure program on functional disability, the measurement instrument has to be able to measure change over time. Then, a property such as responsiveness, the ability of a measurement instrument to detect change over time in the construct to be measured $(43,44)$, becomes increasingly important and should be taken into account. 
Still, at the onset of our RCT, we faced many challenges in selecting measurement instruments. Although in the international literature instruments were described and validation studies had been performed, our choices were often very limited because these existing instruments were not available in Dutch language. Hence, before the start of the RCT, a validation study of the FOPQ-C was undertaken.

In summary, there is a growing availability of different validated measurement instrument for chronic pain in children and adolescents both on pain and non-pain domains. This allows investigators to choose measurement instruments that are relevant and fit for purpose in future studies of treatment effectiveness. Although additional validation efforts in different chronic pain population is still needed, many ingredients are there to generate high quality evidence in adolescent pain research and care.

\section{Implications for clinical practice}

Research has shown that pain-related fear is common in children and adolescents with chronic musculoskeletal pain. Several studies have demonstrated that the presence of pain-related fear is associated with pain-related disability. The mechanism through which pain-related fear influences pain-related disability is theoretically modelled in the Interpersonal Fear Avoidance Model of adolescent chronic pain. These findings offer opportunities to target pain-related fear through treatment in order to decrease functional disability in adolescents with chronic musculoskeletal pain.

Therefore, it is worthwhile to assess the presence of pain-related fear in adolescents with functional disability, to check whether pain-related fear is involved. One of the measurement instruments that can be used for this purpose is the Fear of Pain Questionnaire. For this questionnaire, a validated Dutch version is now available, that can be used in Dutch speaking adolescents with chronic musculoskeletal pain.

If pain-related fear is indeed present, and the complexity of problems within the family system allow for outpatient rehabilitation treatment, the new interdisciplinary graded exposure program can be the treatment of choice to reduce functional disability by targeting pain-related fear. In the multicentre RCT presented in this dissertation, it was demonstrated that this exposure program, targeting pain-related fear, was more effective in decreasing pain-related functional disability, than care as usual in adolescents with chronic musculoskeletal pain. From the process evaluation it appeared that treatment teams had a favourable opinion about the exposure program and intended to keep working with the program. Some of the treatment teams offering the exposure program worried about long term effects of the exposure program. Concerns about the long term effectiveness of the exposure program have appeared to be unjustified though, as the results even showed long term effectiveness of the exposure program. Even at 10 and 12 months after the start of the intervention, the exposure program was more effective in decreasing functional disability than care as usual. Second, our results considering treatment effectiveness also confirm the conceptual associations described in the underlying theoretical Interpersonal Fear 
Avoidance Model. In the RCT it was confirmed that, when targeting pain-related fear during treatment, the level of functional disability could decrease more as compared to a treatment without a specific focus on pain-related fear.

Based on these results, implementation of the exposure program in usual rehabilitation care seems recommended. An evaluation of the cost-effectiveness of the exposure program is warranted in order to further study the value of the exposure program for rehabilitation care. As to implementation, for treatment teams it is possible to offer the new program after 4 days of training, keeping in mind that the treatment teams expressed a need for individual feedback on their performance and the need to gain real world experience after this training to build confidence. We insist that proper training is being followed before treatment teams start offering graded exposure in vivo within the context of the exposure program.

\section{Recommendations for further research}

In this chapter, suggestions for further research have already been made, such as the evaluation of the cost-effectiveness of the exposure program, evaluation of the treatment fidelity of the parent module and expansion of the assessment of pain-related fear in Dutch setting by translating further questionnaires, to name a few. In addition to these suggestions, other suggestions are offered here.

Although the results of the RCT were in favour of the exposure program in decreasing functional disability as compared to usual care, further research of graded exposure in vivo in adolescents with chronic pain could strengthen our confidence of the effectiveness of this type of treatment for this population. Whether a (multicentre) randomized controlled trial design is then the preferred design of choice can be debated. Still, RCTs are recommended as the gold standard to evaluate treatment effectiveness of interventions. However, the execution of (multicentre) RCTs requires substantial funding and resources for adequate personnel, training and site coordination, which is increasingly difficult to attain. Further, many trials struggle with small sample sizes and may suffer a loss of power when analysing treatment effects (13). Some of these difficulties can be overcome when using an alternative study design, such as a single-case design (45), as has been done in studies investigating effectiveness of graded exposure in vivo in adults (46). In single-case designs a lower sample size is required as each patient functions as its own control, they provide an opportunity for customizing pain treatments, and they are considered to be a more feasible design which is more compatible with clinical practice than an RCT (45). If still choosing for a randomized controlled trial, embracing a pragmatic approach can increase the applicability of the results for clinical practice $(15,19,47)$. Within the pragmatic approach, there is a preference for alternative (active) control groups rather than placebo control groups or waiting list control groups (20), which is most relevant for clinicians and policy makers when choosing between different treatment options.

Besides these methodological considerations for future research, an interesting suggestion has been made with regard to clinical implications for future research. Morley and colleagues (48) have suggested that the psychological profile of adolescents seeking treatment for chronic pain 
may be more important than their pain condition for determining treatment response. In our RCT, it was also assumed (based on the interpersonal fear avoidance model) that a graded exposure program would benefit adolescents reporting pain-related fear. For adolescents reporting no pain-related fear, an exposure program would not be recommended. Categorizing adolescents based on their psychological profile could potentially be a powerful predictor for treatment effect (28), but it may also offer opportunities to prevent exacerbation of chronic pain complaints that eventually result in pain-related disability. When combining a psychological profile with known risk factors and prognostic factors for chronic pain (49), patients may be identified in an earlier stage and we may be able to take measures to prevent disability. This could be an opportunity for primary care. Further, a psychological profile to identify patients that may benefit from certain treatments such as our exposure program may also be an advantage for other patient groups, for example adolescents with certain rheumatic diseases such as juvenile idiopathic arthritis (JIA). In these patients, the level of disability clinically observed is oftentimes larger than expected, based on their disease activity. Currently, recommendations for the management of JIA encompass early and aggressive anti-inflammatory treatment, combining pharmacological and non-pharmacological treatments focussing on the treatment of pain, disability and coping with the disease (50). In line with patients with chronic pain syndromes, patients with rheumatoid arthritis have been identified as susceptible to developing pain-related fear and these fears have been demonstrated to be predictive for functioning $(51,52)$.

\section{CONCLUSION}

Considering the results of the process evaluation and effect evaluation of the randomized controlled trial, the new interdisciplinary graded exposure program can be performed according to protocol, is feasible to implement in rehabilitation care and demonstrates clinically relevant and statistically significant decreases in functional disability in adolescents with chronic musculoskeletal pain reporting pain-related fear. Therefore, it is concluded that this RCT showed that a graded exposure program is a valuable addition to the treatment possibilities available to reduce functional disability in adolescents with chronic musculoskeletal pain reporting pain-related fear. 


\section{REFERENCES}

1. Kashikar-Zuck S, Flowers SR, Claar RL, Guite JW, Logan DE, Lynch-Jordan AM, et al. Clinical utility and validity of the Functional Disability Inventory among a multicenter sample of youth with chronic pain. Pain. 2011;152(7):1600-7.

2. Sil S, Arnold LM, Lynch-Jordan A, Ting TV, Peugh J, Cunningham N, et al. Identifying treatment responders and predictors of improvement after cognitive-behavioral therapy for juvenile fibromyalgia. Pain. 2014;155(7):1206-12.

3. Hechler T, Ruhe AK, Schmidt P, Hirsch J, Wager J, Dobe M, et al. Inpatient-based intensive interdisciplinary pain treatment for highly impaired children with severe chronic pain: randomized controlled trial of efficacy and economic effects. Pain. 2014;155(1):118-28.

4. Lynch ME, Campbell F, Clark AJ, Dunbar MJ, Goldstein D, Peng P, et al. A systematic review of the effect of waiting for treatment for chronic pain. Pain. 2008;136(1-2):97-116.

5. Wiertz C, Goossens M, Spek EM, Verbunt JA. A cognitive-behavioral program for parents of children with chronic musculoskeletal pain; A feasibility study. Eur J Pain. 2017;21(9):1571-81.

6. Leeuw M, Goossens ME, de Vet HC, Vlaeyen JW. The fidelity of treatment delivery can be assessed in treatment outcome studies: a successful illustration from behavioral medicine. J Clin Epidemiol. 2009;62(1):81-90.

7. Goubert L, Simons LE. Cognitive styles and processes in paediatric pain. In: McGrath PA, Stevens BJ, Walker SM, Zempsky WT, editors. Oxford Textbook of Paediatric Pain. Oxford: Oxford University Press; 2014. p. 95-101.

8. Simons LE, Kaczynski KJ. The Fear Avoidance model of chronic pain: examination for pediatric application. The Journal of Pain 2012;13(9):827-35.

9. Simons LE, Smith A, Kaczynski K, Basch M. Living in fear of your child's pain: the Parent Fear of Pain Questionnaire. Pain. 2015;156(4):694-702.

10. Groenewald CB, Palermo TM. The price of pain: the economics of chronic adolescent pain. Pain Manag. 2015;5(2):61-4.

11. Law EF, Groenewald CB, Zhou C, Palermo TM. Impact on Health Care Costs for Adolescents Receiving Adjunctive Internet-Delivered Cognitive-Behavioral Therapy: Results of a Randomized Controlled Trial. J Pain. 2018.

12. Evans JR, Benore E, Banez GA. The Cost-Effectiveness of Intensive Interdisciplinary Pediatric Chronic Pain Rehabilitation. J Pediatr Psychol. 2016;41(8):849-56.

13. Palermo TM, Eccleston C, Lewandowski AS, Williams ACdC, Morley S. Randomized controlled trials of psychological therapies for management of chronic pain in children and adolescents: An updated meta-analytic review. Pain. 2010;148(3):387-97.

14. Kashikar-Zuck SM. Psychological interventions for pediatric chronic pain-the good news and the challenges ahead. Pain. 2010;148(3):361-2.

15. Loudon K, Treweek S, Sullivan F, Donnan P, Thorpe KE, Zwarenstein M. The PRECIS-2 tool: designing trials that are fit for purpose. BMJ. 2015;350:h2147.

16. Patsopoulos NA. A pragmatic view on pragmatic trials. Dialogues Clin Neurosci. 2011;13(2):217-24.

17. Eccleston C, Fisher E, Law E, Bartlett J, Palermo TM. Psychological interventions for parents of children and adolescents with chronic illness. The Cochrane database of systematic reviews. 2015;4:CD009660. 
18. Freedland KE, Mohr DC, Davidson KW, Schwartz JE. Usual and unusual care: existing practice control groups in randomized controlled trials of behavioral interventions. Psychosom Med. 2011;73(4):323-35.

19. Schwartz D, Lellouch J. Explanatory and pragmatic attitudes in therapeutical trials. J Clin Epidemiol. 2009;62(5):499-505.

20. Rowbotham MC, Gilron I, Glazer C, Rice AS, Smith BH, Stewart WF, et al. Can pragmatic trials help us better understand chronic pain and improve treatment? Pain. 2013;154(5):643-6.

21. Taves DR. The use of minimization in clinical trials. Contemp Clin Trials. 2010;31(2):180-4.

22. Scott NW, MCPherson GC, Ramsay CR, Campbell MK. The method of minimization for allocation to clinical trials. a review. Control Clin Trials. 2002;23(6):662-74.

23. Treasure T, MacRae KD. Minimisation: the platinum standard for trials?. Randomisation doesn't guarantee similarity of groups; minimisation does. BMJ. 1998;317(7155):362-3.

24. Yeaton WH, Sechrest L. Critical dimensions in the choice and maintenance of successful treatments: strength, integrity, and effectiveness. J Consult Clin Psychol. 1981;49(2):156-67.

25. Bellg AJ, Borrelli B, Resnick B, Hecht J, Minicucci DS, Ory M, et al. Enhancing treatment fidelity in health behavior change studies: best practices and recommendations from the NIH Behavior Change Consortium. Health Psychol. 2004;23(5):443-51.

26. Lichstein KL, Riedel BW, Grieve R. Fair tests of clinical trials: A treatment implementation model. Advances in Behaviour Research and Therapy. 1994;16(1):1-29.

27. Finley GA, MacLaren Chorney J, Campbell L. Not small adults: the emerging role of pediatric pain services. Can J Anaesth. 2014;61(2):180-7.

28. Fisher E, Heathcote L, Palermo TM, de CWAC, Lau J, Eccleston C. Systematic review and meta-analysis of psychological therapies for children with chronic pain. J Pediatr Psychol. 2014;39(8):763-82.

29. Hechler T, Kanstrup M, Holley AL, Simons LE, Wicksell R, Hirschfeld G, et al. Systematic Review on Intensive Interdisciplinary Pain Treatment of Children With Chronic Pain. Pediatrics. 2015;136(1):115-27.

30. De Vet HC, Terwee CB, Mokkink LB, Knol DL. Measurement in Medicine. Cambridge: Cambridge University Press; 2011.

31. Eccleston C, Jordan AL, Crombez G. The impact of chronic pain on adolescents: a review of previously used measures. J Pediatr Psychol. 2006;31(7):684-97.

32. McGrath PJ, Walco GA, Turk DC, Dworkin RH, Brown MT, Davidson K, et al. Core outcome domains and measures for pediatric acute and chronic/recurrent pain clinical trials: PedIMMPACT recommendations. J Pain. 2008;9(9):771-83.

33. Palermo TM. Assessment of chronic pain in children: current status and emerging topics. Pain research \& management. 2009;14(1):21-6.

34. Fisher E, Heathcote LC, Eccleston C, Simons LE, Palermo TM. Assessment of Pain Anxiety, Pain Catastrophizing, and Fear of Pain in Children and Adolescents With Chronic Pain: A Systematic Review and Meta-Analysis. J Pediatr Psychol. 2018;43(3):314-25.

35. Simons LE, Sieberg CB, Carpino E, Logan D, Berde C. The Fear of Pain Questionnaire (FOPQ): Assessment of Pain-Related Fear Among Children and Adolescents With Chronic Pain. The Journal of Pain. 2011;12(6):677-86.

36. Huguet A, McGrath PJ, Pardos J. Development and preliminary testing of a scale to assess pain-related fear in children and adolescents. The Journal of Pain 2011;12(8):840-8. 
37. Verbunt JA, Nijhuis A, Vikstrom M, Stevens A, Haga N, de Jong J, et al. The psychometric characteristics of an assessment instrument for perceived harmfulness in adolescents with musculoskeletal pain (PHODA-youth). Eur J Pain. 2015;19(5):695-705.

38. Eccleston C, Jordan A, McCracken LM, Sleed M, Connell H, Clinch J. The Bath Adolescent Pain Questionnaire (BAPQ): development and preliminary psychometric evaluation of an instrument to assess the impact of chronic pain on adolescents. Pain. 2005;118(1-2):263-70.

39. Crombez G, Bijttebier P, Eccleston C, Mascagni T, Mertens G, Goubert L, et al. The child version of the pain catastrophizing scale (PCS-C): a preliminary validation. Pain. 2003;104(3):639-46.

40. Walker LS, Smith CA, Garber J, Van Slyke DA. Development and validation of the pain response inventory for children. Psychol Assess. 1997;9(4):392-405.

41. Thastum M, Zachariae R, Scholer M, Herlin T. A Danish adaptation of the Pain Coping Questionnaire for children: preliminary data concerning reliability and validity. Acta Paediatr. 1999;88(2):132-8.

42. Goubert L, Eccleston C, Vervoort T, Jordan A, Crombez G. Parental catastrophizing about their child's pain. The parent version of the Pain Catastrophizing Scale (PCS-P): A preliminary validation. Pain. 2006;123(3):254-63.

43. Mokkink LB, Terwee CB, Patrick DL, Alonso J, Stratford PW, Knol DL, et al. The COSMIN study reached international consensus on taxonomy, terminology, and definitions of measurement properties for health-related patient-reported outcomes. J Clin Epidemiol. 2010;63(7):737-45.

44. Terwee CB, Bot SD, de Boer MR, van der Windt DA, Knol DL, Dekker J, et al. Quality criteria were proposed for measurement properties of health status questionnaires. J Clin Epidemiol. 2007;60(1):34-42.

45. Onghena P, Edgington ES. Customization of pain treatments: single-case design and analysis. Clin J Pain. 2005;21(1):56-68; discussion 9-72.

46. de Jong JR, Vlaeyen JW, Onghena P, Cuypers C, den Hollander M, Ruijgrok J. Reduction of pain-related fear in complex regional pain syndrome type I: the application of graded exposure in vivo. Pain. 2005; 116(3):264-75.

47. Chalkidou K, Tunis S, Whicher D, Fowler R, Zwarenstein M. The role for pragmatic randomized controlled trials (pRCTs) in comparative effectiveness research. Clin Trials. 2012;9(4):436-46.

48. Morley S, Williams A, Eccleston C. Examining the evidence about psychological treatments for chronic pain: time for a paradigm shift? Pain. 2013;154(10):1929-31.

49. Huguet A, Tougas ME, Hayden J, McGrath PJ, Stinson JN, Chambers CT. Systematic review with meta-analysis of childhood and adolescent risk and prognostic factors for musculoskeletal pain. Pain. 2016;157(12):2640-56.

50. Stinson JN, Luca NJ, Jibb LA. Assessment and management of pain in juvenile idiopathic arthritis. Pain research \& management. 2012;17(6):391-6.

51. Strahl C, Kleinknecht RA, Dinnel DL. The role of pain anxiety, coping, and pain self-efficacy in rheumatoid arthritis patient functioning. Behav Res Ther. 2000;38(9):863-73.

52. Tong A, Jones J, Craig JC, Singh-Grewal D. Children's experiences of living with juvenile idiopathic arthritis: a thematic synthesis of qualitative studies. Arthritis Care Res (Hoboken). 2012;64(9):1392-404. 




\section{INTRODUCTION}

The aim of writing this dissertation was to add to the scientific evidence e.g. the knowledge on rehabilitation treatment for adolescents with chronic musculoskeletal pain. The research presented in this dissertation is therefore dedicated to treatment possibilities for adolescents with chronic musculoskeletal pain in rehabilitation care. In previous chapters this research and the findings are described and discussed from a scientific perspective. In this chapter however, the valorisation addendum, the results will be described from a different perspective. Valorisation is the process of creating value from knowledge, by making knowledge suitable and/or available for social (and/or economic) use and by making knowledge suitable for translation into competitive products, services, processes and new commercial activities. Here, the broader societal relevance of the findings of this dissertation will be described as well as to whom our findings are relevant, the products developed and activities for knowledge transfer. In addition, the innovative character of our research will be discussed.

\section{RELEVANCE}

With health care expenditures rising each year, it is very important to search for possibilities to optimize patient care, preferably with lower costs involved as well. In the RCT presented in this dissertation, it was demonstrated that the newly developed interdisciplinary graded exposure program reduced functional disability in adolescents with chronic musculoskeletal pain and pain-related fear. In fact, adolescents in the exposure program on average showed a larger reduction in functional disability than the adolescents in the control group who received care as usual. Besides the added scientific value of these findings, there is clearly a direct benefit at a group level for the adolescents with chronic musculoskeletal pain who participated in this study. The reduction in functional disability allows these adolescents to participate and engage in normal age appropriate activities such as attending school, interacting with friends, and performing a hobby.

Because of the pragmatic approach that was used in the RCT the results are highly applicable to rehabilitation care outside the study setting and therefore these findings are also relevant for other adolescents with chronic musculoskeletal pain reporting pain-related fear. Due to the diversity in rehabilitation centers participating in the study, the study setting is a broad representation of the actual rehabilitation setting in the Netherlands. In the study, two pediatric rehabilitation centers, a rehabilitation department of a general hospital and a rehabilitation department of an academic hospital were represented.

As the consequences of the burden of chronic pain are not only felt by the adolescents themselves, but also by their families and by society as a whole, the findings of this dissertation are relevant to them as well. Parents and the family system are significantly influenced when they care for an adolescent with chronic musculoskeletal pain. Amelioration of the adolescents complaints benefits the parents and families as well. And as society bears the (large) financial consequences of increased health care utilization due to the pain complaints, there is a direct benefit if a treatment results in a reduction of these costs. The costs involved are however not only limited 
to direct and indirect medical costs, but also involve for example productivity losses of parents who care for their adolescent. These costs are of great importance to insurers, policy makers, and employers. Alongside the RCT, data for cost-effectiveness analysis has been collected and will be analyzed in the near future. Results of these analysis can underscore the societal relevance of our research in terms of the costs involved in adolescent chronic pain and treatment.

\section{TARGET GROUPS}

Researchers of chronic pain treatment in adolescents may take an interest in our research findings. However, other people outside academia might be interested in our findings as well. In the first place there are the clinicians who are confronted with adolescents with chronic musculoskeletal pain in their clinical practices and are willing to offer these patients some relief. These are not only the consultants in rehabilitation medicine in specialized rehabilitation care, as these patients are also seen by general practitioners, pediatricians, rheumatologists, neurologists, orthopedists, physiotherapists, occupational therapists, psychiatrists and psychologists to name a few. All of these disciplines could benefit from knowing our findings. They can refer patients efficiently to the exposure treatment if it seems to be a good fit for a patient to help him or her improve functioning. Second, policy makers can use the findings of this dissertation when searching for, and deciding upon evidence based treatment possibilities for adolescent chronic musculoskeletal pain to be included in guidelines. A theory-driven, relatively short and effective intervention has been described in this dissertation. Third, also insurers can be interested in our findings because we demonstrated that an evidence-based treatment for adolescent musculoskeletal chronic pain exists. For all target groups now mentioned, one important question that remains to be answered is the cost-effectiveness of the new interdisciplinary exposure program compared to usual rehabilitation care. Knowledge of both the clinical effectiveness and the cost-effectiveness of the new intervention should be used as a strong argument in decisions about inclusion of this program in guidelines and further implementation of the intervention in clinical rehabilitation practice. As part of our RCT, data on direct and indirect costs have been collected, as well as parental productivity losses and adolescent school absence. This data still needs to be analyzed and can provide insight in the cost-effectiveness of the exposure program. Results on the cost-effectiveness of the program can further confirm the value of the intervention.

\section{PRODUCTS AND KNOWLEDGE-TRANSFER}

When the study on effectiveness of the exposure program was designed, detailed treatment protocols were set up for each separate module of the program, specifying exactly how the interdisciplinary program should be offered to the adolescents and their parents. These treatment protocols were complemented by information booklets and homework assignments for the adolescents and information leaflets and presentation materials for the parent meetings. Further, a three day training was developed to learn treatment teams the techniques of graded exposure in vivo treatment for adolescents with chronic musculoskeletal pain. As a first step in the set-up of the study, three additional treatment teams were educated and trained in offering the exposure 
program. Additionally, the program was implemented in their rehabilitation centers. This involved careful implementation of all program elements and procedures within the existing treatments and schedules of the different rehabilitation centers. As a consequences of these activities, protocols, accompanying materials, and the training are existent and can be used and distributed again in case of further implementation of the program in rehabilitation care. Based on the results of our process evaluation on the interdisciplinary exposure program, these materials will be adjusted to make some further improvements. For example by including the regular intervision meetings that should take place once treatment teams start offering the exposure in their treatment center and offering guidance on how to conduct these meetings. The center of expertise in pain rehabilitation Adelante/Maastricht University is currently in the process of refining this training to enable regular training possibilities for new treatment teams and implementation of the treatment in new centers in the future.

Second, to facilitate knowledge-transfer to professionals, we published a book in the Dutch language in 2017 on graded exposure, a cognitive behavioral treatment of chronic pain: 'Graded Exposure, een cognitief gedragsmatige aanpak van chronische pijn' (Editors prof. dr. J.A. Verbunt, and prof. dr. R.E.J.M. Smeets). In this book, all knowledge on graded exposure that has been collected over the past 30 years is incorporated to be used by therapists and physicians who want to offer this treatment to patients with chronic pain. The book describes the application of exposure in both adults with various types of chronic pain and adolescents, but elaborates as well on the logistics involved in implementing the treatment and the training of the treatment teams. The training of the treatment teams is of vital importance when the treatment is implemented in a new center. Without good training high quality delivery of the treatment is a challenge and it is less likely that the successes of this method described in the scientific papers on this treatment are equaled. An essential part of this training is the recognition of pain-related fear in the patient during the screening phase in order to determine whether exposure is the right choice of treatment. Therapists need to learn how to recognize this fear when it is present, since patients often do not express their fears in an explicit manner. Measurement instruments such as described below can help. These measurement instruments are available for other treatment teams interested and can bought or will be provided upon request.

Third, a Dutch version of the Fear of Pain Questionnaire child version was created and validated, as no measure to assess the presence of fear of pain or activity avoidance existed in the Dutch language at the moment this study was commenced. Later, the Photograph Series of Daily Activities for Youth was published as well, a measure for perceived harmfulness of activities, which is a related construct. These two measures allow for identification of fear of pain and activity avoidance due to pain-related fear and can help determine whether an exposure in vivo treatment would be suited for a particular patient. Both measurement instruments therefore provide a unique and valuable opportunity in the selection of a treatment that is likely to offer the greatest benefit to adolescents with chronic musculoskeletal pain and pain-related fear. Good use of the PHODA-Youth was also part of the training that treatment teams received at the beginning of our RCT. These instruments are available in Dutch language and were found to have good psychometric properties. Again, these positive results from scientific studies confirm that these instruments can be used in rehabilitation setting for the benefit of the patients. 


\section{INNOVATION}

The findings presented in this dissertation have an explicit innovative character, as it is one of the first studies in a randomized design on the effectiveness of interdisciplinary program in a population of adolescents with chronic pain and the first on the specific topic of graded exposure for this population of patients. Although graded exposure in vivo had been applied successfully in various adult populations with different types of chronic pain, differences between adults and adolescents required the execution of a new scientific study in this younger population. Based on previous research it was hypothesized that graded exposure in vivo treatment could be successful in reducing functional disability in adolescents as well, and the research presented in this dissertation confirms that hypothesis.

\section{REALIZATION}

To spread the word on exposure treatment for adolescents with chronic pain and pain-related fear, there are different possibilities for different people. The research team will spread the evidence that emerged from our studies through scientific publications and presentations on (inter) national congresses and symposia for researchers and health care professionals. Our results will be presented on the 17 World Congress on Pain in September 2018 and on the Dutch Congress of Rehabilitation Medicine in November 2018. Further, the Dutch working group for children and adolescents with chronic pain and fatigue can provide an important contribution in informing rehabilitation centers about the treatment and advising policy makers on the incorporation of the exposure program in guidelines.

As proper training in exposure is very important, training of new treatment teams is recommended to be organized in a controlled manner. Offering exposure to a patient is not just a matter of copying another therapist, but involves education and continued practice and evaluation. Therefore training should only be offered by skilled and qualified trainers in order to guarantee high quality training for new teams. In my opinion, besides the content of the program, training should also involve guidance in the implementation process of the exposure treatment in a new rehabilitation center. Further, when newly incorporating program trajectories for patients, a monthly intervision meeting for the treatment team members offering the program should be planned as well.

\section{SUMMARY}

This chapter described the broader societal relevance of the findings presented in this dissertation. In the first place, our findings are relevant for adolescents with chronic musculoskeletal pain, their parents, and health care providers in rehabilitation care, who can benefit directly from the effective exposure program that was investigated. Additionally, other health care providers, policy makers and insurers can use our results to choose a suitable treatment program for adolescents with chronic musculoskeletal pain and pain-related fear. To facilitate knowledge transfer of our findings, the protocols and training that were developed can be adjusted and used for further 
implementation. Moreover, the book 'Graded Exposure' is now available for health care providers that summarizes all knowledge gathered in the last 30 years on exposure treatment for both adults and adolescents. Even measurement instruments, such as the PHODA-Youth and Fear of Pain Questionnaire - Dutch version are now available for use in Dutch adolescent rehabilitation care. In conclusion, we have demonstrated with this first study of its kind, that graded exposure is an effective treatment program for adolescents with chronic pain. Now we need to spread the word so that all patients in need of this treatment can benefit. 



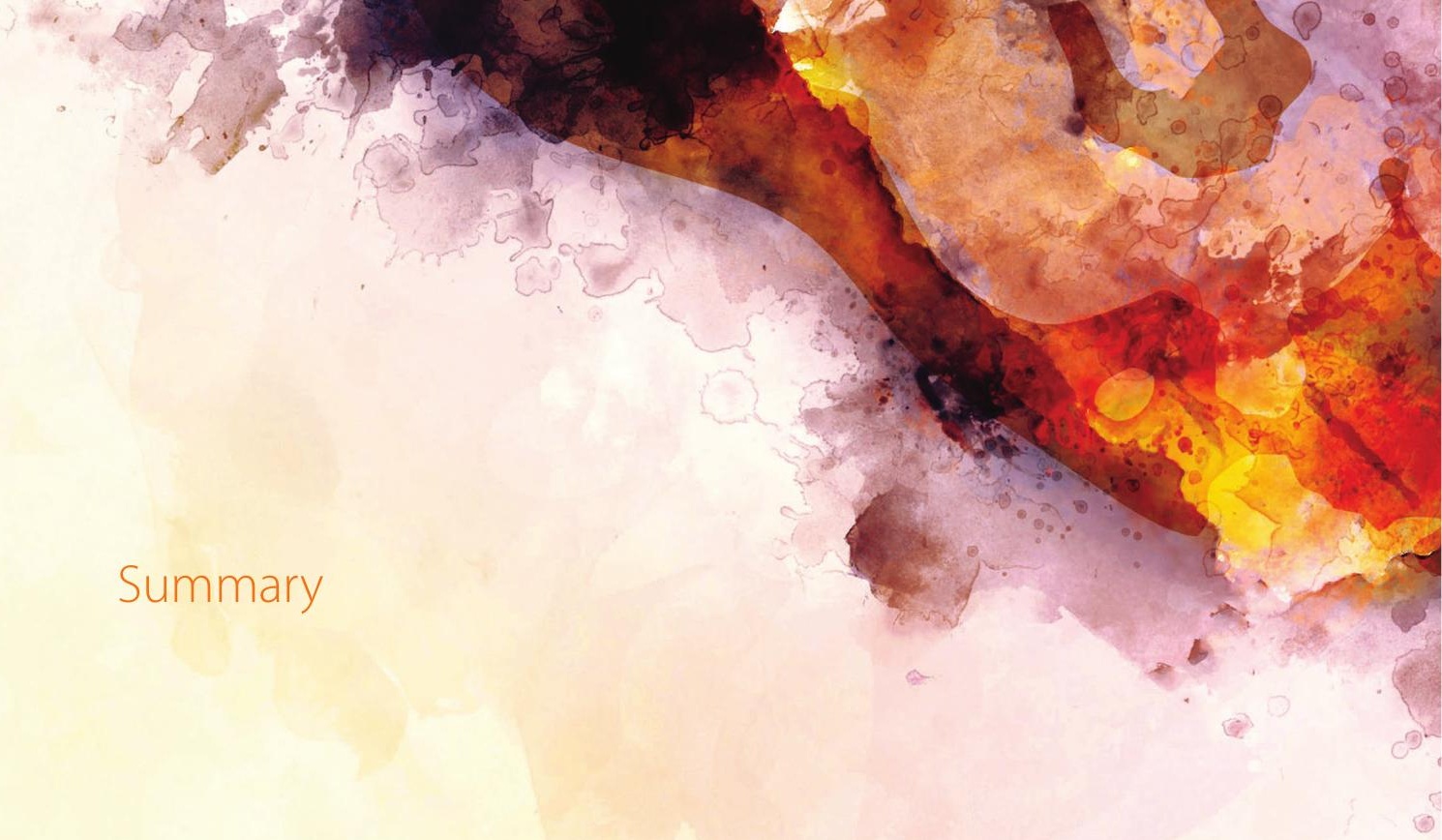





\section{SUMMARY}

Chronic pain in children and adolescents is a common problem, affecting up to $25 \%$ of Dutch school children ( $0-18$ years). With regard to the severity of the pain complaints, it has been reported that $5 \%$ of schoolchildren (8-16 years) have moderate to severe chronic pain problems that cause high pain intensity and moderate to high pain-related disability. Chronic musculoskeletal pain is one of the most reported chronic pain conditions in youth, next to chronic headache and chronic abdominal pain, and is most prevalent in older children and adolescents (8-18 years). Chronic musculoskeletal pain is defined as pain of a musculoskeletal origin and includes limb pain, back pain, Complex Regional Pain Syndrome, Complaints in Arm, Neck and Shoulder, Whiplash Associated Disorder, fibromyalgia, and joint hypermobility syndrome. Despite the high prevalence of this type of chronic pain, research on treatment possibilities for this population is relatively restricted. However, in the latest years, efforts have been made to develop theoretical insights into the working mechanisms for the development and maintenance of adolescent chronic musculoskeletal pain. This research also revealed opportunities to target treatment approaches. The focus of this dissertation is on treatment of adolescents with chronic musculoskeletal pain with a duration of complaints of at least 3 months.

For children and adolescents (8-18 years) with chronic musculoskeletal pain the negative consequences that are often reported are changes in social functioning such as less contact with peers, disturbances in school functioning and school absence, changes in general emotional well-being, increased levels of anxiety and depression. Specifically the detrimental effect of pain-related fear on pain-related disability in adolescents is increasingly recognized. It has been estimated that fear of pain accounts for $40 \%$ of the variance in pain-related disability. Therefore, one of the objectives of this dissertation was to develop an intervention specifically targeting pain-related fear in order to decrease pain-related disability.

As a result of emerging evidence on the role of pain-related fear in youth, the interpersonal fear avoidance model was developed in 2012. This model describes how pain can cause disability through activity avoidance due to pain-related fear and catastrophic thinking about pain. Compared with the original fear avoidance model for adults, the interpersonal fear avoidance model incorporates the social system in which the pain of the adolescent exists, expressed as the interaction with parents. In adults, graded exposure in vivo is an effective treatment to improve functioning. Graded exposure in vivo is a cognitive behavioral treatment that targets pain-related fear and disability through exposing patients to activities and situations previously avoided because of fear of pain or re-injury. Specifically for adolescents with chronic musculoskeletal pain, an interdisciplinary graded exposure program was developed to be offered in outpatient specialized rehabilitation care. The exposure program consists of an adolescent module and a module for parents. The exposure program was designed as an individual treatment for adolescents, whereas the module for parents was intended to be offered in a group setting. The interdisciplinary treatment team offering the program was led by a consultant in rehabilitation medicine, and further consisted of a psychologist, and a physiotherapist or occupational therapist. The parent module 
of the exposure program was developed to involve the family system as part of the treatment of the adolescent. In addition, the exposure program for adolescents also included an additional physical training for adolescents diagnosed with joint hypermobility syndrome.

The main aim of this dissertation is to add to the scientific evidence on rehabilitation treatment for adolescents with chronic musculoskeletal pain, with the ultimate goal to ameliorate negative consequences of their complaints and increase their quality of life. The efforts towards reaching this goal consisted of the performance of a multicenter randomized controlled trial to study the effectiveness of the interdisciplinary exposure program compared with usual care in four Dutch rehabilitation centers. Further, the psychometric properties of a measurement instrument to identify pain related fear in Dutch adolescents have been studied.

Chapter 2 describes the study protocol of a pragmatic randomized controlled trial that was designed to evaluate the effectiveness of a newly developed interdisciplinary graded exposure program for adolescents with chronic musculoskeletal pain compared with usual rehabilitation care in four Dutch rehabilitation centers. A pragmatic approach was chosen to ensure that results obtained from this study are most applicable to rehabilitation practice. Usual rehabilitation care is generally an interdisciplinary graded activity treatment that uses a time-contingent stepwise increase of the adolescents' activity levels to reduce functional disability. Adolescents between 12-21 years old, with chronic musculoskeletal pain, referred to outpatient rehabilitation treatment were eligible to participate together with their parents. Self-reported measurements were performed at baseline, and at 2, 4, 10 and 12 months after start of treatment. The primary outcome measure is functional disability, measured with the functional disability inventory. Secondary outcome measures are fear of pain, catastrophizing, perceived harmfulness, pain intensity, depressive symptoms and quality of life. Further, total direct and indirect costs and health-related quality of life will be measured. A process evaluation was designed to evaluate protocol adherence, patient centeredness of the exposure program and adolescent treatment expectations.

The results of the effect evaluation of the randomized controlled trial are presented in Chapter 3. Recruitment of participants was between august 2014 and September 2016, with a 12-month follow up. Data was analyzed by intention to treat linear mixed model analysis. Data was analyzed of in total 53 adolescents, predominantly female with a mean age of 16 years. Adolescent in the exposure program showed a clinically relevant and statistically significant decrease in functional disability compared with care as usual at all time points. For the secondary measures, only for perceived harmfulness a significant mean difference was found between the two treatment groups at all time points. Further, protocol adherence by the treatment teams in the exposure program was high and contamination of the intervention by the alternative intervention (contamination of the exposure program by usual care and vice versa) was below the threshold and therefore considered absent. Based on these results, it was concluded that in adolescents with chronic musculoskeletal pain and pain related fear, the exposure program leads to a clinically relevant and statistically significant larger decrease in functional disability than usual care does. Furthermore, results on protocol adherence and contamination imply an honest comparison between the two interventions. 
In Chapter 4, the focus is on the quality of the delivery of the interdisciplinary graded exposure program as it was offered to the adolescents by the treatment teams of the four participating treatment centers. The objective of this study was to evaluate in greater detail whether the exposure program for adolescents with chronic musculoskeletal pain reporting pain related fear, was performed according to protocol (treatment fidelity), and whether it is feasible to implement the program in rehabilitation care. In this process evaluation, quantitative and qualitative data on participant characteristics (adolescents, parents and therapists), attendance and participants' opinion on the program were collected by means of registration forms, questionnaires and group interviews. To evaluate treatment fidelity, audio and video recordings of program sessions were collected and analyzed by two independent raters.

Thirty adolescents were offered the exposure program, of which 23 started the program. Adolescents attended on average $90 \%$ of the treatment sessions. At least one parent per adolescent participated in the program. Analysis of 20 randomly selected recordings of treatment sessions revealed that treatment fidelity of the adolescent module was high, since on average $81 \%$ of essential treatment elements were offered to the adolescents in the preparation phase, education phase and treatment phase of the program. Therefore, it was concluded that the interdisciplinary exposure program has been performed largely according to protocol. Adolescents considered the delivery of the program to be client-centered and parents considered the delivery of the program to be family-centered. Treatment teams expressed a favorable opinion about the exposure program and intend to implement the program within their rehabilitation centers. Therefore, implementation of the program is considered feasible in specialized rehabilitation care. Although treatment teams were trained for four days, they perceived good practice of the program challenging. In the future, the organization of regular intervision-sessions within the centers can help to ensure high quality program delivery. Based on the results of the randomized controlled trial it was concluded that the exposure program is effective in reducing functional disability and is feasible to be offered. Therefore, implementation of the program in rehabilitation care for adolescents with chronic musculoskeletal pain and pain-related fear is recommended.

Since pain-related fear is an important construct in the development and maintenance of chronic pain, it is essential to measure this construct carefully. The Fear of Pain Questionnaire (child version), originally developed in the USA, assesses pain-related fear in children and adolescents with chronic pain, and demonstrates sound psychometric properties. Chapter $\mathbf{5}$ reports on the translation into Dutch language, and evaluation of psychometric properties of this measure for fear of pain and activity avoidance.

A cross-sectional study was performed in which after forward and backward translation of the original Fear of Pain Questionnaire (Child version) 86 adolescents (aged 11-22 years old) with chronic musculoskeletal pain completed an assessment containing the Dutch Fear of Pain Questionnaire, and questionnaires about demographics, pain catastrophizing, functional disability and pain intensity. Exploratory factor analysis resulted in a two-factor structure explaining $43 \%$ of the variance that was labeled a Fear of Pain subscale and an Avoidance of Activities subscale. Internal consistency was strong. Hypothesis testing to evaluate construct validity resulted in five out of six hypotheses that were confirmed about the direction and strength of the correlation between the constructs measured by the Fear of Pain Questionnaire child version and comparative constructs 
(pain catastrophizing, functional disability and pain intensity). It was concluded that the Dutch version of the fear of pain questionnaire child version demonstrated good internal consistency and good construct validity in a population of adolescents with chronic musculoskeletal pain. Therefore, the Dutch questionnaire can be used in rehabilitation care to identify the presence of pain-related fear in Dutch speaking adolescents with musculoskeletal chronic pain.

In Chapter $\mathbf{6}$ a general discussion on the main findings of this dissertation is provided. The main aim of this dissertation was to add to the scientific evidence on rehabilitation treatment for adolescents with chronic musculoskeletal pain, with the ultimate goal to ameliorate negative consequences of the adolescent's complaints and to increase their quality of life. It was concluded that the main study of this dissertation showed that an interdisciplinary graded exposure program is a valuable addition to the treatment possibilities available to reduce functional disability in adolescents with chronic musculoskeletal pain reporting pain-related fear. Furthermore, a Dutch version of the Fear of Pain Questionnaire child version was found to be useful for identification of pain-related fear in adolescents with chronic musculoskeletal pain.

To our knowledge this was the first randomized controlled trial evaluating the effectiveness of an interdisciplinary graded exposure program targeting pain-related fear in order to decrease functional disability in adolescents with chronic musculoskeletal pain. Due to a pragmatic approach in the RCT, the results of this trial are generalizable and highly applicable to daily rehabilitation practice, the setting in which the program ultimately is intended to be offered. Still, there were methodological challenges that had to be dealt with, especially in the randomized controlled trial.

One of the major problems in randomized controlled trials in children and adolescents with chronic pain is their relatively small trial size with the risk of low power when analyzing the data. Also in our case the desired 124 participants for the study were not attained. However, due to a larger mean difference in the primary outcome measure between the interventions, the power of our analyses is adequate. Furthermore, many previous performed randomized controlled trials evaluating treatment effects use waiting-list control or treatment as usual as control conditions. Choosing an active care as usual control intervention best suited our aim of designing an effectiveness trial. Further, measurement instruments form a basis in diagnosis, prognosis and evaluation of treatment in care and research in adolescent chronic pain. At the onset of the research presented in this dissertation, choosing appropriate and high quality measurement instruments was a challenge. However, advances have been made in the past years, for example in the development of theoretical models that allow for theory-driven treatment development and also theory driven selection of measurement instruments, or development of instrument measuring constructs such as pain-related fear, or recognition of parental influences, reflected in the development of instruments for parents or instruments with both an adolescent and a parent version. Taking the interpersonal fear-avoidance model into account, it is recommended to translate and validate additional fear of pain questionnaire to enable a full assessment of the presence of fear of pain in the family system of the adolescent. The Parent Fear of Pain Questionnaire measures parent pain-related fears in the context of their adolescents pain, and the Fear of Pain question- 
naire Parent report could be used as a parent proxy report to assess pain-related fear in children and adolescents. For this last questionnaire, data on a Dutch translation has been collected and analysis is planned.

Currently, there are no solid estimates of the economic burden of adolescent chronic pain, although this information can be very useful for policy makers. Furthermore, little is known about potential cost savings by treatment of prevention of chronic pain. Very few studies have been performed outside the Netherlands. These studies, however, showed that the costs are substantial and that treatment can result in savings mainly in reduced health care utilization and reductions in parental missed work. Future studies are recommended to compare for example the cost-effectiveness of the exposure program to usual care in order to determine the economic value of the exposure program for rehabilitation care. Also in our randomized controlled trial, data for an economic evaluation of the new exposure program compared with usual care have been collected an can be analyzed to determine the cost-effectiveness of these interventions.

Finally, further research of graded exposure in vivo for adolescents with chronic pain could strengthen our confidence in the effectiveness of this type of treatment for this population. Researchers can consider single-case designs in order to overcome the challenge of small sample sizes in randomized controlled trials and increase the feasibility of performing a study. However, when still opting for a randomized trial design, a pragmatic approach will provide results that are most relevant for clinicians and policy makers for choosing between options of care. 



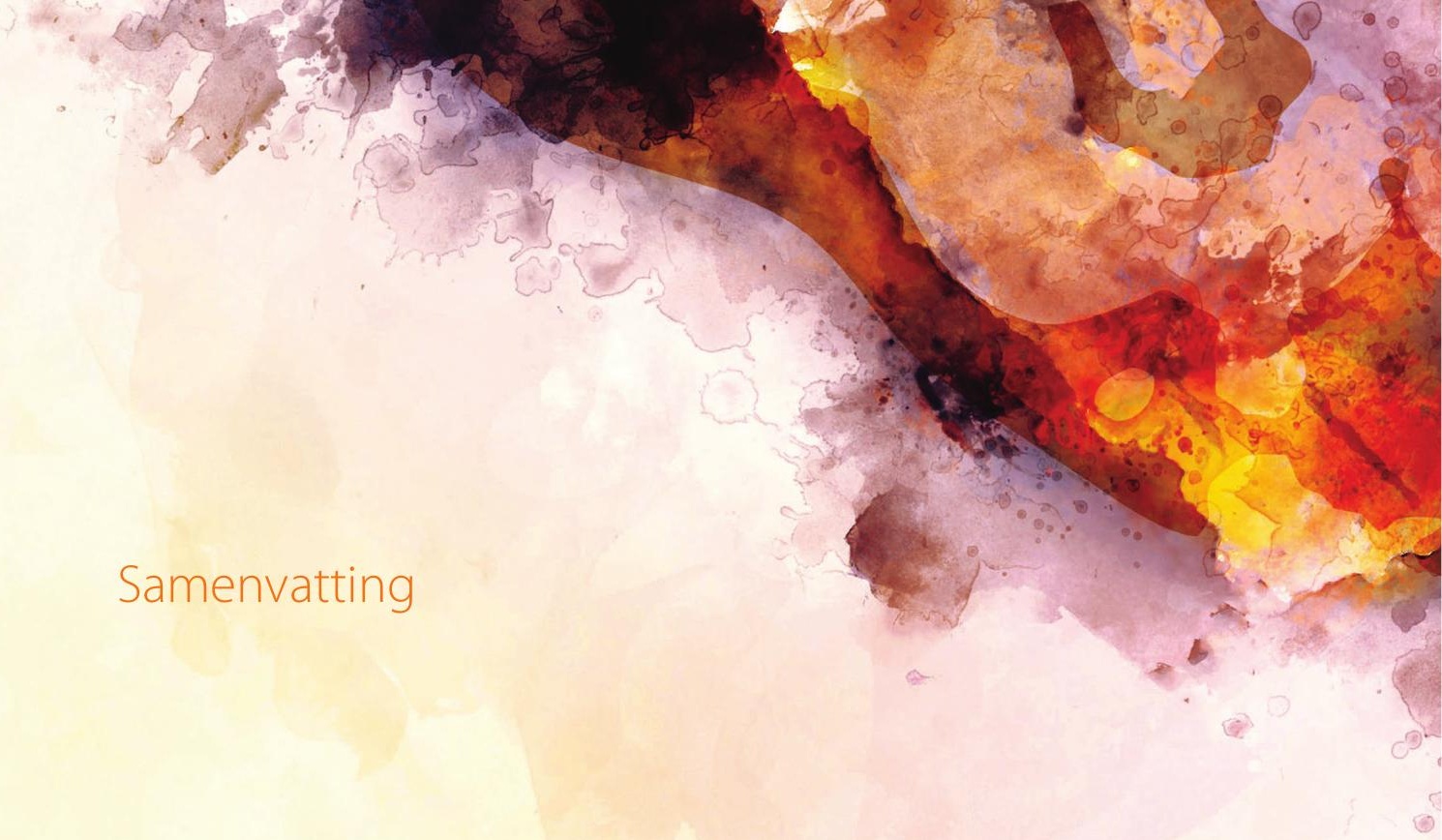





\section{SAMENVATTING}

Chronische pijn bij kinderen en adolescenten is een veelvoorkomend probleem waarmee naar schatting 25\% van de Nederlandse schoolkinderen wordt geconfronteerd. Met betrekking tot de ernst van de chronische pijn is gerapporteerd dat bij 5\% van de schoolkinderen (8-16 jaar) de pijnintensiteit hoog is en leidt tot matige en ernstige pijn gerelateerde beperkingen. Chronische musculoskeletale pijn is een van de meest voorkomende soorten chronische pijn bij de jeugd, naast chronische hoofdpijn en chronische buikpijn, en heeft de hoogste prevalentie bij oudere kinderen en adolescenten (8-18 jaar). Deze chronische pijn wordt gedefinieerd als pijn met een musculoskeletale oorzaak, waaronder pijn in de ledematen, rugpijn, complex regionaal pijnsyndroom, klachten aan arm, nek en schouder, whiplash, fibromyalgie en hypermobiliteitssyndroom. Ondanks dat deze vorm van pijn relatief vaak voorkomt is nog maar relatief weinig onderzoek gedaan naar hoe we deze jongeren kunnen behandelen. In de afgelopen jaren is steeds meer onderzoek verricht naar de theorie en de werkingsmechanismen van het ontstaan en in stand houden van chronische musculoskeletale pijn bij adolescenten. Uit dit onderzoek kwamen aangrijpingspunten voor behandeling naar voren. De focus van dit proefschrift ligt op behandeling van adolescenten met chronische musculoskeletale pijn bij wie de pijn langer dan 3 maanden aanwezig is.

De gevolgen van de chronische musculoskeletale pijn voor kinderen en adolescenten (8-18 jaar) die vaak worden gerapporteerd zijn veranderingen in sociaal functioneren zoals een verminderd contact met vrienden, verminderd functioneren op school, schoolverzuim, veranderingen in algemeen emotioneel welbevinden, verhoogde angst en depressie. Met name de negatieve invloed van de pijn gerelateerde angst op de beperkingen van de jongeren wordt steeds vaker aangetoond. Naar schatting wordt $40 \%$ van de variantie in beperkingen veroorzaakt door pijn gerelateerde angst. Een van de doelen van dit proefschrift was daarom het ontwikkelen van een interventie die zich specifiek richt op pijn gerelateerde angst om pijn gerelateerde beperkingen te verminderen.

Als gevolg van de groeiende kennis over de rol van pijn gerelateerde angst bij jongeren verscheen in 2012 het interpersoonlijk vrees-vermijdingsmodel. Dit model beschrijft hoe pijn beperkingen kan veroorzaken als gevolg van het vermijden van activiteiten vanwege pijn gerelateerde angst en catastroferende gedachten over de pijn. Vergeleken met het originele vrees-vermijdingsmodel voor volwassenen is binnen het interpersoonlijk vrees-vermijdingsmodel het sociale systeem meegenomen waarbinnen de pijn van de jongeren bestaat, met name de interactie met de ouders. Bij volwassenen is graded exposure in vivo effectief gebleken in het verbeteren van het functioneren. Graded exposure in vivo is een cognitieve gedragsbehandeling gericht op pijn gerelateerde angst en beperkingen door patiënten bloot te stellen aan activiteiten en situaties die zij normaliter vermeden vanwege pijn-gerelateerde angst of angst voor schade. Speciaal voor jongeren met chronische musculoskeletale pijn is dit programma vertaald naar een interdisciplinair graded exposure programma dat in poliklinische revalidatiezorg kan worden aangeboden. Het exposure programma is ontwikkeld als een individuele behandeling voor adolescenten, waarbij de ouders een aparte module in groepsverband wordt aangeboden. Het behandelteam bestaat uit de revalidatiearts als hoofd van het behandelteam, de psycholoog en de fysiotherapeut of ergotherapeut. De oudermodule van 
het exposure programma is ontwikkeld om ook het systeem te betrekken bij de behandeling van de adolescent. Voor jongeren die gediagnosticeerd zijn met het hypermobiliteitssyndroom wordt de module voor adolescenten uitgebreid met een fysieke training.

Het primaire doel van dit proefschrift is om bij te dragen aan het wetenschappelijk bewijs over revalidatiebehandelingen voor adolescenten met chronische musculoskeletale pijn. Met als uiteindelijk doel om de negatieve gevolgen van de pijnklachten van deze jongeren te verminderen en hun kwaliteit van leven te verbeteren. Een gerandomiseerde gecontroleerde studie naar het effect van het graded exposure programma in vergelijking met de gebruikelijke zorg is uitgevoerd in vier revalidatiecentra. Daarnaast is ook de bruikbaarheid en psychometrische eigenschappen van een meetinstrument gericht op het in kaart brengen van pijn gerelateerde angst onderzocht.

In hoofdstuk 2 wordt het studieprotocol voor de pragmatische gerandomiseerde gecontroleerde studie beschreven. Deze studie is ontwikkeld om de effectiviteit van het nieuw ontwikkelde interdisciplinaire graded exposure programma voor adolescenten met chronische musculoskeletale pijnklachten te vergelijken met de gebruikelijke revalidatiezorg in vier Nederlandse revalidatiecentra. Er is gekozen voor een pragmatische benadering van deze studie om te zorgen dat de resultaten het meest bruikbaar zijn voor de revalidatiepraktijk. Gebruikelijke revalidatiezorg is voornamelijk een interdisciplinaire graded activity behandeling waarbij op een stapsgewijze tijd-contingente manier het activiteitenniveau van de adolescent wordt verhoogd om functionele beperkingen te verminderen. Adolescenten tussen 12 en 21 jaar met chronische musculoskeletale pijn en een verwijzing naar poliklinische revalidatiebehandeling kwamen in aanmerking voor deelname samen met hun ouders. Vragenlijsten werden door de jongeren en ouders ingevuld voor aanvang van de behandeling (basislijn) en 2, 4, 10 en 12 maanden na start van de behandeling. De primaire uitkomstmaat van de studie is functionele beperkingen, gemeten met de 'functional disability inventory'. Secundaire uitkomstmaten zijn angst voor pijn, catastroferen, ervaren schadelijkheid, pijnintensiteit, symptomen van depressie en kwaliteit van leven. Verder werden ook totale directe en indirect kosten en gezondheidsgerelateerde kwaliteit van leven gemeten. Er werd een procesevaluatie ontworpen om te onderzoeken hoe goed de therapeuten zich hielden aan het behandelprotocol en hoe patiëntgericht het exposure programma was. Daarbij werd ook gekeken naar de verwachtingen die adolescenten hadden ten aanzien van de behandeling.

De resultaten van het onderzoek naar de effectiviteit van het nieuwe exposure programma in de gerandomiseerde gecontroleerde studie zijn beschreven in hoofdstuk 3. Tussen augustus 2014 en september 2016 zijn jongeren geworven voor deelname aan deze studie, waarbinnen zij 12 maanden zijn gevolgd. De data is geanalyseerd volgens 'intention to treat linear mixed model'analyse. In totaal is de data van 53 adolescenten geanalyseerd, voornamelijk van vrouwen met een gemiddelde leeftijd van 16 jaar. Bij de jongeren in het exposure programma was een klinisch relevante en statistisch significante vermindering in functionele beperkingen zichtbaar vergeleken met de jongeren in de gebruikelijke zorg op alle meetpunten. Voor de secundaire uitkomstmaten was er alleen voor ervaren schadelijkheid een statistisch significant verschil tussen de groepen op alle meetpunten. Daarnaast bleek dat de therapeuten die het exposure protocol hebben aangeboden zich in hoge mate gehouden hadden aan het behandelprotocol en dat de behandelingen vrij waren van elementen uit de behandeling waar deze mee vergeleken werd (contaminatie - Er 
waren geen exposure elementen aanwezig in de gebruikelijke zorg en omgekeerd). Op basis van deze resultaten werd geconcludeerd dat bij jongeren met chronische musculoskeletale pijn en pijn gerelateerde angst het exposure programma tot een klinisch relevante en statistisch significante vermindering in functionele beperking leidt vergeleken met gebruikelijke zorg. Daarnaast impliceren de resultaten over het aanbieden van de behandeling volgens het behandelprotocol en contaminatie dat er een eerlijke vergelijking is gemaakt tussen beide behandelingen.

In hoofdstuk 4 ligt de focus op de kwaliteit van de aangeboden interdisciplinaire graded exposure behandeling door behandelteams van de vier deelnemende behandelcentra. Het doel van deze studie was om in meer detail te onderzoeken of het exposure programma volgens protocol werd aangeboden aan de adolescenten met chronische musculoskeletale pijnklachten en pijn gerelateerde angst. Daarnaast werd gekeken hoe haalbaar het was om het exposure programma te implementeren in de revalidatiesetting. Voor deze procesevaluatie werden kwantitatieve en kwalitatieve data verzameld over deelnemerskenmerken (adolescenten, ouders, behandelaren), aanwezigheid en de meningen over de behandeling van adolescenten, ouders en behandelaren. Deze data werd verzameld op registratieformulieren, op vragenlijsten en in groepsinterviews. Om de integriteit van de aangeboden behandeling te onderzoeken werden audio en video opnames gemaakt van behandelsessies die vervolgens door twee onafhankelijke beoordelaars werden geanalyseerd.

Aan 30 adolescenten werd het exposure programma aangeboden. Drieëntwintig adolescenten zijn met het programma gestart. Deze jongeren hebben 90\% van de behandelsessies gevolgd. Minimaal 1 ouder per adolescent nam deel aan de oudermodule. Uit analyse van 20 willekeurig geselecteerde opnames van de behandelsessies bleek dat de behandelintegriteit hoog was. Uit de opnamen bleek dat gemiddeld $81 \%$ van de essentiële behandelelementen aan de adolescenten werd aangeboden in de intake-, educatie- en behandelfase van het programma. Er werd daarom geconcludeerd dat het exposure programma voornamelijk volgens protocol is aangeboden. Adolescenten vonden het programma cliënt-gericht en ouders vonden het programma familie-gericht. Behandelteams hadden ook een positieve mening over het programma en hebben de intentie om het exposure programma in hun centrum te implementeren. Mede hierdoor wordt implementatie van het exposure programma als haalbaar beoordeeld voor de revalidatiesetting. Hoewel behandelteams een training van vier dagen hebben gevolgd vonden ze het toch een uitdaging om het programma goed aan te bieden. In de toekomst kunnen intervisie-bijeenkomsten binnen de behandelcentra helpen om de kwaliteit van het aanbieden van de behandeling te waarborgen. Op basis van de resultaten van de gerandomiseerde gecontroleerde studie wordt geconcludeerd dat het exposure programma effectief is in het verminderen van functionele beperkingen en dat het haalbaar is om dit programma aan te bieden. Daarom wordt implementatie van het programma in de revalidatiezorg voor adolescenten met chronische musculoskeletale pijn en pijn gerelateerde angst aanbevolen.

Omdat pijn gerelateerde angst een belangrijk construct is in het ontwikkelen en in stand houden van chronische pijn bij jongeren, is het zeer essentieel om dit construct goed in kaart te brengen. De oorspronkelijk in de Verenigde Staten ontwikkelde 'Fear of Pain Questionnaire' (kind versie) bleek goede psychometrische eigenschappen te hebben om pijn-gerelateerde angst bij 
kinderen en adolescenten met chronische pijn te meten. Hoofstuk 5 beschrijft de Nederlandse vertaling en onderzoek van de psychometrische eigenschappen van dit meetinstrument voor pijn gerelateerde angst en vermijdingsgedrag.

Een dwarsdoorsnede onderzoek werd uitgevoerd waarin na vertaling en terugvertaling van de originele vragenlijst 86 Nederlandse adolescenten (11-22 jaar) met chronische musculoskeletale pijnklachten een aantal vragenlijsten invulden, waaronder de Nederlandse 'Fear of Pain Questionnaire,' vragen over demografische kenmerken, pijn catastroferen, functionele beperkingen en pijnintensiteit. Uit een explorerende factor analyse bleek een twee-factor structuur die $43 \%$ van de variantie kon verklaren. De factoren kregen de labels 'fear of pain'- subschaal en 'avoidance of activities'- subschaal. Interne consistentie was hoog. Voor het onderzoeken van de constructvaliditeit werden zes hypotheses geformuleerd over de richting en de sterkte van de correlatie tussen het construct gemeten door de Fear of Pain vragenlijst en de constructen waarmee vergeleken werd (catastroferen, functionele beperkingen en pijnintensiteit). Vijf van de zes hypotheses kon worden bevestigd. Er werd geconcludeerd dat de interne consistentie van de Nederlandse versie van de 'Fear of Pain' vragenlijst kind versie hoog is en dat de constructvaliditeit goed is. Daarom kan de Nederlandse vragenlijst worden gebruikt in de revalidatiesetting voor het identificeren van pijn gerelateerde angst bij Nederlands sprekende jongeren met musculoskeletale pijn.

In hoofdstuk $\mathbf{6}$ worden in een algemene discussie de belangrijkste bevindingen van dit proefschrift beschreven. Het belangrijkste doel van dit proefschrift was om bij te dragen aan de wetenschappelijke kennis over revalidatiebehandeling voor adolescenten met chronische musculoskeletale pijn. Met als uiteindelijke doel om de negatieve consequenties van de pijnklachten te verlichten en de kwaliteit van leven te verbeteren. Er werd geconcludeerd dat de belangrijkste studie van dit proefschrift liet zien dat een interdisciplinair graded exposure programma een waardevolle aanvulling is op de behandelmogelijkheden voor jongeren met chronische musculoskeletale pijnklachten en pijngerelateerde angst. Daarnaast is de Nederlandse versie van de 'Fear of Pain'vragenlijst een bruikbaar meetinstrument voor het identificeren van pijn gerelateerde angst bij jongeren met chronische musculoskeletale pijn.

Voor zo ver wij weten is dit de eerste gerandomiseerde gecontroleerde studie naar de effectiviteit van een interdisciplinair graded exposure programma gericht op pijn gerelateerde angst met als doel om functionele beperkingen bij jongeren met chronische musculoskeletale pijn te verminderen. Vanwege de pragmatische opzet van de studie zijn de resultaten generaliseerbaar en zeer van toepassing op de dagelijkse revalidatiezorg, de setting waarin de nieuwe behandeling uiteindelijk zou moeten worden aangeboden. Met name in de gerandomiseerde gecontroleerde studie waren er echter een aantal methodologische uitdagingen waar wij mee om moesten gaan.

Een van de grote problemen bij gerandomiseerde gecontroleerde studies bij kinderen en adolescenten met chronische pijn is dat het relatief kleine studies zijn, waardoor het risico op een lage power bij de analyse van de data bestaat. Ook in onze studie werd het gewenste aantal van 124 deelnemende jongeren niet gehaald. Maar door een groter gemiddeld verschil tussen beide groepen op de primaire uitkomstmaat was er bij onze analyses geen powerprobleem. Daarnaast hebben veel gerandomiseerde gecontroleerde studies die gekeken hebben naar effecten van 
behandelingen gebruik gemaakt van wachtlijst controle of gebruikelijke zorg controle-behandelingen. Het kiezen van een actieve gebruikelijke zorg controle behandeling paste het best bij ons doel om naar behandeleffectiviteit (zoals deze in de praktijk zou zijn) te kijken.

Meetinstrumenten vormen de basis voor het vaststellen, voorspellen en evalueren van behandelingen bij jongeren met chronische pijn. Bij aanvang van ons project was het een grote uitdaging om geschikte meetinstrumenten te kiezen met een goede kwaliteit. De afgelopen jaren is echter wel vooruitgang geboekt op dit gebied. Bijvoorbeeld door de ontwikkeling van theoretische modellen wordt het ontwikkelen van behandelingen die op theorie zijn gebaseerd mogelijk en dat maakt het ook mogelijk om meetinstrumenten te kiezen op basis van deze theorie. Ook zijn er meetinstrumenten ontwikkeld voor het meten van constructen zoals pijn-gerelateerde angst. Verder heeft het erkennen van de invloed van ouders geleid tot het ontwikkelen van meetinstrumenten specifiek voor ouders of van meetinstrumenten waarvoor zowel een ouder als een kind-versie bestaat. Kijken naar het interpersoonlijk vrees-vermijdingsmodel wordt dan ook aanbevolen om ook andere 'Fear of Pain' vragenlijsten te vertalen en de kwaliteit te onderzoeken om op deze manier een omvattende meting van angst voor pijn te kunnen doen binnen het systeem van de adolescent met chronische pijn. De 'Parent Fear of Pain Questionnaire meet pijn-gerelateerde angst bij ouders in de context van de pijnklachten van hun adolescent. De 'Fear of Pain questionnaire ouder versie kan gebruikt worden als een proxy maat voor het meten van angst voor pijn van de adolescent. Voor deze laatste vragenlijst is data verzameld voor een Nederlandse versie van deze vragenlijst. Deze data moet nog worden geanalyseerd.

Op dit moment is er geen duidelijke schatting van de economische impact van chronische pijn bij adolescenten. Deze informatie kan zeer bruikbaar zijn voor beleidsmakers. Verder is ook maar weinig bekend over potentiële besparingen door behandeling van chronische pijn of preventie hiervan. Slechts zeer weinig studies zijn uitgevoerd op dit gebied, allen buiten Nederland. Deze studies lieten echter wel zien dat de kosten substantieel zijn en dat behandeling kan leiden tot besparingen met name in het gebruik van gezondheidszorg en in een vermindering van de productiviteitsverliezen van ouders die niet naar het werk gaan. Het wordt aanbevolen dat toekomstige studies zich richten op het vergelijken van bijvoorbeeld de kosteneffectiviteit van het exposure programma en de gebruikelijke zorg om de economische waarde van het exposure programma voor de revalidatiesetting te bepalen. Ook in onze gerandomiseerde gecontroleerde studie is de data verzameld die nodig is voor het uitvoeren van een economische evaluatie om de kosteneffectiviteit van het nieuwe exposure programma te bepalen.

Tot slot kan verder onderzoek naar exposure in vivo voor adolescenten met chronische pijn het vertrouwen doen groeien in de effectiviteit van dit type behandeling in deze populatie. Onderzoekers kunnen een single-case design overwegen als alternatief voor een gerandomiseerde gecontroleerde studie om op die manier de uitdaging van de studiegrootte te omzeilen en om de haalbaarheid van het uitvoeren van deze studie te vergroten. Wordt er toch gekozen voor een gerandomiseerde gecontroleerde studie, dan leidt de keuze voor een pragmatische benadering tot resultaten die het meest relevant zijn voor clinici en beleidsmakers die moeten kiezen tussen verschillende behandelingen. 



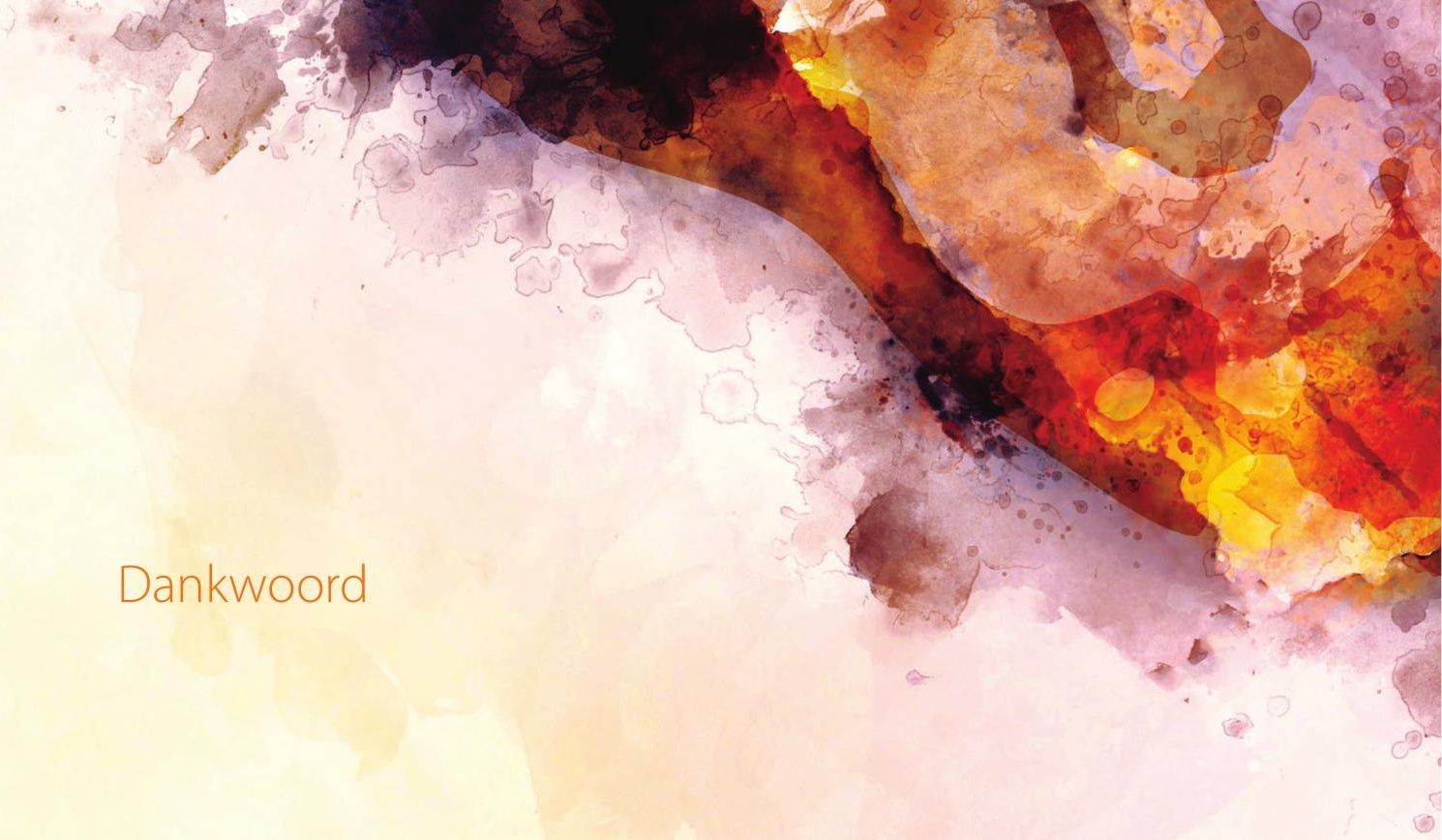





\section{DANKWOORD}

Na vijf jaar is hier het moment dat mijn promotietraject wordt afgerond. Met het boekje dat hierbij tot stand is gekomen is een deel van dit traject tastbaar geworden. Het overgrote deel van dit boekje gaat over de inhoud van dit traject en de resultaten die het onderzoek heeft opgeleverd. In dit hoofdstuk ligt de aandacht op een heel ander deel van het promotietraject, namelijk de mensen om mij heen die het uitvoeren van het promotietraject voor mij mogelijk hebben gemaakt. De mensen, zowel in werk- als privésfeer, die ik enorm waardeer om hun bijdrage, in welke vorm dan ook. De mensen met wie ik herinneringen heb gemaakt in de afgelopen vijf jaar, met wie ik ervaringen heb gedeeld, op wie ik kon bouwen en steunen wanneer dit nodig was, de mensen die naast mij hard hebben gewerkt om het onderzoek te laten lopen, kortom, de mensen zonder wie ik dit proefschrift nooit had kunnen schrijven.

Bij het schrijven van dit dankwoord komen ook weer talloze herinneringen boven en realiseer ik dat vijf jaar een lange periode is waarin veel is gebeurd. Daarom hoop ik dat ik niemand vergeten ben in dit hoofdstuk. Mocht dit toch zo zijn, alsnog heel hartelijk dank.

Allereerst wil ik mijn dank uitspreken naar mijn promotieteam. Jeanine, Mariëlle en Carolien, jullie stonden vijf jaar lang voor mij klaar. Wat was ik trots op een promotieteam waarin verschillende disciplines (revalidatiegeneeskunde, psychologie, epidemiologie) verenigd waren met zowel klinische en wetenschappelijke ervaring. Het gaf mij vertrouwen om zo'n kundig en ervaren team achter mij te hebben staan. Jullie brachten alle drie verschillende perspectieven ter sprake in discussies, wat ik zeer verrijkend vond voor de artikelen die hier uiteindelijk uit voortgekomen zijn. Jullie boden mij veel vrijheid in de vormgeving van mijn traject, gaven mij kansen en daar heb ik veel van kunnen leren. Ik heb veel bewondering en respect voor de wijze waarop jullie, zelfs in avonduren, weekenden en vanuit het buitenland, alles op alles hebben gezet om het project zo goed mogelijk te laten verlopen en mij hierin te begeleiden. Ook wanneer de (privé)omstandigheden moeilijk waren.

Marion, jij kwam voor de start van de werving van de patiënten ons onderzoeksteam versterken. Je was voor mij absoluut onmisbaar voor alle data-verzameling en verwerking binnen de RCT. Je hield samen met mij een oogje op het reilen en zijlen van alle lopende onderzoekszaken. Ik durf geen lijst te beginnen van alle klussen waar jij je binnen mijn project mee bezig hield, bang er een aantal te vergeten, maar je bijdrage was heel divers. Daarnaast was je een hele fijne collega om mee samen te werken. Ik kon jou alles vragen, je gaf mij vertrouwen dat het werk wat in jouw handen was goed gebeurde. Heel veel dank voor alles. Margareth, ook jouw deur stond altijd open. Ik kon bij je terecht met al mijn vragen. Ook voor een gesprekje over hoe het allemaal ging. Je maakte menig collega enthousiast, waaronder ook mij, om toch echt even een half uurtje te wandelen in de pauze. Jouw voorbeeld deed volgen! Mijn overige fijne collega's op de vakgroep, ook hartelijk dank, voor jullie interesse en collegialiteit. Mijn ervaring is dat jullie een hele hulpvaardige groep collega's zijn, bij wie ik mij thuis voelde op het werk. Het invoeren van het gezamenlijke koffiemoment vond ik een fijne zet, zodat we elkaar ook wat regelmatiger gezamenlijk zagen. 
Onmisbaar voor het uitvoeren van de RCT waren alle coördinatoren, artsen, therapeuten en planners van de vier verschillende revalidatiecentra die mee hebben gewerkt aan dit project; team Maastricht, team Roermond, team Breda en team Rotterdam. Jullie hebben een enorme prestatie geleverd, door de nieuwe exposure behandeling te leren, te implementeren en daadwerkelijk patiënten te werven en behandelen voor 2B Active. Ook de therapeuten van Adelante in Houthem en van Maasgouw, die bereid waren om voor de controleconditie in Maastricht, naar Maastricht te reizen om hier behandeling aan te bieden, hartelijk dank voor jullie inzet. Zonder het enthousiasme en het doorzettingsvermogen getoond door deze groepen hadden wij de RCT niet succesvol kunnen uitvoeren. Een bijzondere vermelding nog voor het team van Maastricht, die gewerkt hebben aan de ontwikkeling van het exposure behandelprotocol voor jongeren, het ouderprogramma en die de training van de behandelteams op de overige locaties hebben verzorgd. Natuurlijk gaat ook mijn dank uit naar alle jongeren en hun ouders die ons het vertrouwen schonken door aan onze studie mee te werken. Ongetwijfeld een spannend besluit. Ik ben vol dankbaarheid en respect dat jullie deze stap hebben genomen, behandeling binnen 2B Active zijn gestart en met zo veel geduld telkens de vragenlijsten hebben ingevuld. Zonder jullie was de uitvoering van de RCT natuurlijk ook onmogelijk geweest.

Niet te vergeten zijn ook mijn co-auteurs, die met kritische blik de manuscripten voor de verschillende publicaties hebben gelezen en van kritische feedback hebben voorzien. Dit was heel leerzaam voor mij. Mede door jullie inzet heeft het proefschrift, zoals het hier nu ligt, vorm gekregen.

De beoordelingscommissie Prof. dr. Annelies Boonen, Prof. dr. Madelon Peters, Prof. Dr. Raymond Ostelo en Dr. rer. nat. Julia Wager; hartelijk dank voor de tijd en moeite die jullie hebben genomen voor het lezen en beoordelen van dit proefschrift.

In mijn persoonlijke kring kan ik niet om mijn vrienden en vriendinnen heen. Jullie hebben de afgelopen tijd weinig van mij gehoord of hebben mij weinig gezien. Dank voor jullie geduld en begrip. Het was fijn dat wij een leuke tijd hadden die paar momenten dat wij elkaar wel zagen en het was fijn dat ik mijn verhaal met jullie mocht delen. Dan wil ik ook mijn volleybalteam graag bedanken. Volleybal is mijn grootste hobby, waar ik in de eerste drie jaren van mijn traject minimaal 3 keer per week tijdens training en wedstrijd alles van het promoveren los kon laten. Het trainen, spelen en de gezelligheid van het team was voor mij vaak de uitlaatklep die ik nodig had om het allemaal vol te houden.

Reni, wij begonnen in de zomer van 2013 vrijwel tegelijk ons promotietraject. We gingen vrijwel dezelfde weg en kwamen beren op de weg tegen van dezelfde soort. Wat heb ik een lieve en fijne collega aan jou gehad. Je optimisme en vrolijkheid aan het bureau tegenover mij had ik niet kunnen missen. Je concentratie, enthousiasme en gedrevenheid in je werk waren voor mij echt een voorbeeld. Ik denk met een goed gevoel terug aan de vele liters thee die wij inderdaad hebben gedronken, de 'challenges' die wij zijn aangegaan op de kamer om de lange kantoordagen wat op te breken met lichamelijke activiteit en de nevenactiviteiten die we samen hebben georganiseerd. Het was heerlijk om zo'n fijne vriendin als collega te mogen hebben. Ik mocht 11 maanden geleden paranimf zijn bij jouw promotie en ben ontzettend trots dat jij op mijn dag ook naast mij staat. Als kersverse moeder welteverstaan! 
Janneke, ik wil jou in het bijzonder bedanken, als vriendin en als collega. Wij leerden elkaar kennen toen Jeanine ons aan elkaar koppelde voor het uitvoeren van de valideringsstudie van een aantal vragenlijsten. Maar naast dat wij het erg goed met elkaar konden vinden op het gebied van ons werk zie ik jou ook als goede vriendin. We hebben een aantal leuke symposia en congressen samen bijgewoond, waar we leuk en kritisch met elkaar konden discussiëren. Ik ben heel dankbaar dat ik met jou en je collega mee mocht reizen naar Boston voor het Wereld Pijncongres 2018. Met- en door jou gingen een heel aantal nieuwe deuren voor mij open.

Mijn lieve familie. Jullie staan altijd voor mij klaar. Altijd kreeg ik een hand hulp als ik deze nodig had, gevraagd en ongevraagd. Jullie stelden mij nooit teleur. En wat een geduld hebben jullie moeten opbrengen als ik weer eens alles voor mijn werk aan de kant schoof! Dank jullie wel voor alle steun. Nog te meer toen ik vaak een beroep op jullie deed om op te passen op kleine Robin in de laatste schrijffase van het proefschrift. Het voelt goed dat Robin bij zulke lieve mensen terecht kon, zodat ik de ruimte kreeg om dit werkstuk af te maken. Lieve Maartje, jij wees mij er regelmatig op als de balans tussen familie en werk zoek raakte. En jij weet waar je het over hebt met jouw enorme creatieve en muzikale projecten. Je hebt de durf om hierin eerlijk je gevoel te uiten, waar ik veel waardering voor heb. Ik ben je enorm dankbaar dat ook jij tijdens de verdediging als paranimf naast mij staat.

Een laatste dankjewel, maar zeker niet minder welgemeend, gaat naar mijn lieve gezin. Rick, toen ik jou leerde kennen zorgde jij dat ik eens wat anders zag dan studieboeken en de sporthal. En dat doe jij nog steeds. Jij hebt mij de afgelopen vijf jaar alle ruimte gegeven tijdens mijn promotietraject en gesteund daar waar je kon. Soms betekende dat ook dat je er juist voor zorgde dat we eens een stuk gingen wandelen of dat we een film samen keken. Samen hebben we ook heel hard gewerkt aan het kopen van ons eerste huis, de verbouwing van dak tot kruipruimte, onze bruiloft en de komst van onze lieve kleine Robin. Daar waar ik even tekort schoot vulde jij aan, zodat we alles voor elkaar kregen. Dat was niet altijd even gemakkelijk. Met jou kan ik de wereld aan. Lieve Robin, net toen de laatste schrijffase van mijn proefschrift er aan kwam, werd jij geboren. Wat is het fijn dat jij bij ons in het gezin gekomen bent. Jou komst was voor mij een grote motivatie om door te zetten bij die laatste zware loodjes. Klein manneke, wat maak jij ons leven ongelofelijk veel mooier! 
Denkend aan promoveren

Zie ik talloze ideeën

Als circusdieren

Achter tralies staan,

Eindeloos wachten

Tot ooit op een dag

Een van die kooien

Voor hen open zal gaan

En dan in beweging

De vrijheid geschonken

Kunnen groeien

Gebonden aan een hand,

Soms een meegaand

Soms een weerbarstig

Maar altijd een

Onlosmakelijk verband

Van een paard

En zijn ruiter

Als twee muzieknoten

In een akkoord,

Schitterend in de ring

Verrijkend voor velen

Met trots gepresenteerd

Verwacht en gehoord

Carolien Dekker 


\section{About the author}

Carolien Dekker was born in Landgraaf, the Netherlands, on September 28 1988. Following her high school education she started the bachelor Health Sciences at Maastricht University in 2008. During this study she did a major in Health Policy and Management and a minor in Health Promotion and Health Education. In this period, she was awarded a Top 3\% Award twice. This award indicated that she performed amongst the best 3\% of students attending Maastricht University. Carolien obtained her bachelor degree with distinction in 2011.

After her bachelor, Carolien continued her education at Maastricht University and specialized in health sciences research through the Health Sciences Research Master from 2011-2013. In the second year of this Master she performed a full-time research internship at the department of Epidemology at Maastricht University. The internship was completed with a thesis on infant motor development assessment. Next to her studies, Carolien worked as a tutor and trainer in the Health Sciences Bachelor and Health Sciences Research Master at Maastricht University. Carolien graduated for her master with distinction in 2013.

Directly after her graduation, Carolien started her PhD project at the Department of Rehabilitation Medicine at Maastricht University. She was supervised by Prof. Dr. Jeanine Verbunt, Dr. Marielle Goossens, and Dr. Caroline Bastiaenen. Her project focused on outpatient rehabilitation treatment for adolescents with chronic pain. Throughout her PhD trajectory, Carolien gained experience in multiple research activities from design through ethical approval, execution, analysis and reporting of scientific knowledge. In addition, Carolien continued her role as tutor and trainer in the Health Sciences Bachelor and Health Sciences Research Master. She followed several additional courses to further develop as a researcher, participated in writing grant proposals, and presented her work at different national and international conferences. 


\section{PUBLICATIONS}

\section{Peer reviewed publications}

Dekker C, Goosens MEJB, Bastiaenen CHG, Verbunt JAMCF. Study protocol for a multicentre randomized controlled trial on effectiveness of an outpatient multimodal rehabilitation program for adolescents with chronic musculoskeletal pain (2B Active). BMC Musculoskelet Disord. 2016;17:317. doi:10.1186/s12891-016-1178-5.

Dekker C, Bastiaenen CHG, de Vries JE, Simons LE, Goossens MEJB, Verbunt JAMCF. Dutch version of the Fear of Pain Questionnaire for adolescents with chronic pain. Disabil Rehabil. 2017:1-7. doi :10.1080/09638288.2017.1289255.

de Vries JE, Dekker C, Bastiaenen CHG, Goossens MEJB, Engelbert RHH, Verbunt JAMCF. The Dutch version of the self-report Child Activity and Limitations Interview in adolescents with chronic pain. Disabil Rehabil. 2017:1-7. doi:10.1080/09638288.2017.1407969.

\section{Submitted publications}

Dekker C, van Haastregt, JCM, Verbunt JAMCF, de Jong J, van Meulenbroek T, Pernot HFM, et al. Pain-related fear in adolescents with chronic musculoskeletal pain: Process evaluation of an interdisciplinary graded exposure program.

Dekker C, Goossens MEJB, Winkens B, Remerie SC, Bastiaenen CHG, Verbunt JAMCF. Functional disability in adolescents with chronic pain: comparing an interdisciplinary exposure program to usual care, A randomized clinical trial.

\section{Other publications}

Dekker C, Goossens MEJB, Bastiaenen CHG, Verbunt JAMCF. Chapter 16 Onderzoek naar graded exposure voor jongeren. In Verbunt, JA, Smeets, RJEM. (Red). Graded Exposure, 2017. doi:10.1007/978-90-368-1106-4_16 